\title{
Fully developed isotropic turbulence: nonperturbative renormalization group formalism and fixed point solution
}

\author{
Léonie Canet $^{1}$, Bertrand Delamotte ${ }^{2}$, and Nicolás Wschebor ${ }^{2,3}$ \\ ${ }^{1}$ LPMMC, Université Joseph Fourier Grenoble-Alpes, \\ CNRS UMR 5493, 38042 Grenoble Cedex, France \\ ${ }^{2}$ LPTMC, CNRS UMR 7600, Université Pierre et Marie Curie, 75252 Paris Cedex 05, France \\ ${ }^{3}$ Instituto de Física, Facultad de Ingeniería, Universidad de la República, J.H.y Reissig 565, 11000 Montevideo, Uruguay
}

\begin{abstract}
We investigate the regime of fully developed homogeneous and isotropic turbulence of the NavierStokes (NS) equation in the presence of a stochastic forcing, using the nonperturbative (functional) renormalization group (NPRG). Within a simple approximation based on symmetries, we obtain the fixed point solution of the NPRG flow equations that corresponds to fully developed turbulence both in $d=2$ and $d=3$ dimensions. Deviations to the dimensional scalings (Kolmogorov in $d=3$ or Kraichnan-Batchelor in $d=2$ ) are found for the two-point functions. To further analyze these deviations, we derive exact flow equations in the large wave-number limit, and show that the fixed point does not entail the usual scale invariance, thereby identifying the mechanism for the emergence of intermittency within the NPRG framework. The purpose of this work is to provide a detailed basis for NPRG studies of NS turbulence, the determination of the ensuing intermittency exponents is left for future work.
\end{abstract}

PACS numbers: 47.10.ad,47.27.Gs,47.27.ef,05.10.Cc

\section{INTRODUCTION}

The statistical theory of turbulence is more than seventy years old and, despite intensive efforts, it remains unsatisfactory. In $d>2$, two length scales play a dominant role in the phenomenology of fully developed homogeneous and isotropic turbulence: the microscopic (Kolmogorov) scale $\eta$ where energy is dissipated by molecular viscosity and the macroscopic integral scale $L$ where energy is injected in the system. These two scales delineate the inertial range, where energy is conserved and transferred towards the small scales in an energy cascade. Within the inertial range, the equal-time velocity correlation functions exhibit universal scaling, that is, they behave as power laws with exponents independent of the precise mechanisms of energy injection and dissipation. These observations lead to the celebrated K41 scaling theory, proposed by Kolmogorov in 1941 [1 3]. The energy flux constancy relation was derived, which yields the exact "four-fifth law" for the three-velocity correlator. K41 also predicts power-law behaviors for all the correlation functions. Although the experimentally measured energy spectrum and low-order structure functions are well described by K41 theory, systematic deviations from K41 scalings were observed for higher-order correlation functions [4, 5]. Calculating these exponents beyond K41 theory remains a great challenge in the study of fully developed turbulence. In $d=2$, two inertial ranges were predicted to coexist by Kraichnan [6] as a consequence of the conservation of both energy and enstrophy (squared vorticity). In two-dimensional turbulence, part of the energy is transferred from the integral scale to the larger scales in an inverse energy cascade until it is eventually dissipated at the boundaries of the system, while enstrophy flows towards the smaller scales in the direct cascade until it is dissipated at the molecular scale [6, 7]. In the direct cascade, the exponents of the structure functions are also believed to deviate from dimensional scalings [4, 8] and their calculation remains a challenging issue.

This situation appears frustrating if compared to that of critical phenomena occurring at equilibrium, which share many common features with turbulence (e.g. scaling, chaos, universality) 9] and where renormalization group (RG) has led in most cases to a clear understanding of the physics at play and to accurate determinations of the critical exponents [10]. An essential difference is that, in standard equilibrium critical phenomena, a finite set of anomalous dimensions suffices to describe the scaling behavior of all the correlation functions, which is no longer true for turbulence. The correlation functions do exhibit power laws, but each with its specific exponent, which generates multiscaling, or multifractality, and constitutes one of the imprints of turbulence [4]. This phenomenon, generically referred to as intermittency, is investigated in this paper using nonperturbative (functional) renormalization group (NPRG).

Prior to giving an overview of existing RG approaches to describe fully developed homogeneous and isotropic turbulence, let us introduce the relevant microscopic model, which is the Navier-Stokes equation with forcing:

$$
\partial_{t} v_{\alpha}+v_{\beta} \partial_{\beta} v_{\alpha}=-\frac{1}{\rho} \partial_{\alpha} p+\nu \nabla^{2} v_{\alpha}+f_{\alpha}
$$

where the velocity field $\vec{v}$, the pressure field $p$, and the stochastic forcing $\vec{f}$ depend on the space-time coordinates $(t, \vec{x})$, and with $\nu$ the kinematic viscosity and $\rho$ the density of the fluid. Since we aim at studying the turbulent steady state, the presence of the stirring force $\vec{f}$ is essential to balance the dissipative nature of the (unforced) NS equation which otherwise leads to the decay of the velocity fields. We consider in the following incompress- 
ible flows, satisfying

$$
\partial_{\alpha} v_{\alpha}=0 .
$$

We focus on the properties of the turbulent fluid within the inertial range of wavenumbers $p$ corresponding to $L^{-1} \ll p \ll \eta^{-1}$. In this regime, the steady-state correlation functions are expected to be universal in the sense that they do not depend on the precise form of the macroscopic forcing (as long as its Fourier transform is peaked around wave-numbers of the order $L^{-1}$ ). This universality allows one, instead of choosing a deterministic forcing, to take averages on various smooth forcings with an essentially arbitrary probability distribution, as long as the typical scale of the forcing remains the prescribed integral scale. One can hence conveniently choose the simplest of probability distributions: a Gaussian one with zero mean and variance

$$
\left\langle f_{\alpha}(t, \vec{x}) f_{\beta}\left(t^{\prime}, \vec{x}^{\prime}\right)\right\rangle=2 \delta\left(t-t^{\prime}\right) N_{L^{-1}, \alpha \beta}\left(\left|\vec{x}-\vec{x}^{\prime}\right|\right) .
$$

This correlator is local in time, which is crucial to preserve Galilean invariance, and it is centered, in Fourier space, on the inverse of the integral scale $L$. The precise profile of $N_{L^{-1}, \alpha \beta}(x)$, which should not affect universal properties in the inertial regime, will be specified in the following.

As in critical phenomena, scaling is observed in turbulence when the microscopic scale (the Kolmogorov scale $\eta$ ) is sent to zero and the macroscopic one (the integral scale $L$ ) to infinity. In this limit, the expansion parameter, the Reynolds number, diverges. Field theoretic techniques such as renormalization group (RG) are designed to handle the large scale fluctuations developing in strongly correlated systems and we briefly review in the following some of the former attempts in turbulence (for reviews, see [11 13]).

The difficulty when applying RG in turbulence is not so much that the Reynolds number diverges when the ultra-violet (UV) scale $\eta^{-1}$ is sent to infinity. Naively, one could think that the Reynolds number is the expansion parameter in a perturbative treatment, and its divergence would render odd a perturbative analysis based on it. However, it is well known [10] that once the RG is employed, the proper expansion parameter is not the bare Reynolds number but a renormalized parameter obtained from its RG evolution in the long-distance limit, such that the perturbative analysis can work. The real difficulty is to find a situation where the renormalized expansion parameter is small. For standard critical systems, this is achieved around the upper critical dimension $d_{c}$ and a double expansion in the coupling constant and $\epsilon=d_{c}-d$ renders the perturbative expansion well defined. As for turbulence, there is no upper critical dimension but a formal (second) expansion parameter $\epsilon$ can be defined through the forcing profile $N_{L^{-1}, \alpha \beta}(p) \propto p^{4-d-2 \epsilon}$ where $p$ is the wave-number [14 17]. Typically, as explained above, $N_{L^{-1}, \alpha \beta}(p)$ is not a power-law in Fourier space, but is instead sharply peaked around the infra-red (IR) scale $L^{-1}$. One can show that the physical situation is recovered only in a precise limit, eg. when $\epsilon \rightarrow 2$ (in $d=3$ ) or $\epsilon \rightarrow 3$ (in the direct cascade in $d=2$ ). On the other hand, for $\epsilon=0$, the theory is exactly renormalizable and a fixed point of order $\epsilon$ is found in any dimension [18, 19] (the $d=2$ case being particular [20 26]). The challenge for the perturbatively renormalized theory is therefore to extend the results obtained for $\epsilon \rightarrow 0$ to $\epsilon=2$ (or 3) which is far from trivial: The difficulties encountered are very severe and have, up to now, hindered real progress, at least for the calculation of multiscaling behavior in the NS problem [18, 19]. This seems to be related to the appearance of operators with negative critical dimension at finite $\epsilon$.

Let us emphasize that intermittency also occurs in the simpler Kraichnan's passive scalar model [27] where a scalar field is advected by a prescribed Gaussian random field decorrelated in time and self-similar in space. In contrast with Navier-Stokes turbulence, the anomalous exponents for the Kraichnan model have been determined under several controlled approximations including an $\epsilon$ expansion (with $\epsilon$ related to the power spectrum of the noise) or a $1 / d$-expansion, and numerical simulations 28 37] (see [38, 39] for reviews). In this model, composite operators with negative critical dimensions, called "dangerous" operators, were identified and the anomalous exponents could be calculated from their critical dimensions [36, 40]. The calculations were performed at three loops [41, 42] and yielded reasonable results, under mild assumptions on the series behavior. Unfortunately, the same methodology does not seem to work satisfactorily for the Navier-Stokes equation. In this case no operator with negative critical dimension could be identified at infinitesimal $\epsilon$ and thus, the $\epsilon$ expansion does not seem to provide a reliable framework to compute anomalous exponents [18].

Let us notice that another perturbative approach, almost ignored in the subsequent literature, does not rely on an $\epsilon$-expansion but on a self-consistent determination of the quadratic part of the action around which perturbation theory is performed [43]. This approach, after elimination of what is named the "sweeping effect" (the sweeping of the smaller scales by the larger) leads to the existence of an UV attractive fixed point from which, performing an Operator Product Expansion (OPE), can be computed the multiscaling exponents. They turn out to be quite accurate at least for the $n<10$ first equal-time correlation functions of the velocity differences.

A rather different field-theoretic approach, not based on RG, has been developed by L'vov, Procaccia and collaborators. To get rid of the sweeping effect that leads to severe IR singularities in renormalized perturbation theory, these authors use "quasi-Lagrangian" variables instead of the Eulerian velocities [4]. They show that the correlation functions of the differences of these variables are finite order by order in perturbation theory in both limits where the UV and IR scales are removed. 
As a consequence, Kolmogorov scaling holds at all finite orders of the perturbation theory. The only way out of this hindrance is to resum infinite classes of Feynman diagrams. The authors indeed show that these resummations produce new singularities in terms of the integral scale which is therefore the proper renormalization scale [45 55]. They are then able to compute approximately the small order multiscaling exponents in terms of the first one [56].

An alternative RG approach that has been very successful in the study of critical systems either at or out of equilibrium, is the nonperturbative renormalization group (NPRG), which is a modern version of the RG à la Wilson [57 62]. In addition to avoiding many problems encountered perturbatively, such as the need of explicit resummation of IR singularities or the asymptotic nature of the renormalized series [10], this approach has led not only to very accurate [62 69] but also fully nonperturbative [70 75] results in many systems. Of course, approximations are also unavoidable in the NPRG context and they are not always easy to justify when they are not controlled by a small parameter. A very inspiring case is the Kardar-Parisi-Zhang (KPZ) equation describing the stochastic growth of interfaces [76], which is equivalent to Burgers equation in the context of fluids, and which shows fully nonperturbative behavior in the rough phase [77]. Contrary to the standard perturbative RG which fails to all orders in perturbation to find the relevant fixed point and the associated scaling behavior [78], the NPRG approach captures the strong coupling physics of the KPZ equation at and above one dimension [79 83]. One of the aims of this article is to show how this method can be implemented to study Navier-Stokes turbulence.

Let us now turn to other works on turbulence using similar functional RG methods [84 88]. During the publication process of this manuscript, Kraichnan's model has been studied using NPRG methods in 88]. The leading corrections to the exponents of the structure functions have been computed within this framework and they reproduce the known results, which provides an independent validation of the method.

As for the NS problem, the settings developed in Refs. 84] and [86] are closely related to the one presented here. The relevant fixed point for turbulence was already found in $d=3$ in Ref. 84]. However, important elements concerning the symmetries, and multiscaling, were not identified in this early work, and we here bring them out. In particular, the regularization scheme chosen in Ref. 84] prevented from addressing the $d=2$ case. In contrast, the formalisms of Ref. [86] and of the present work cure this problem and enable one to study both bidimensional and tridimensional turbulence within a unified framework. The main difference between the work of Ref. [86] and ours is that the former focuses on power-law forcing whereas we consider a forcing localized at a definite external or integral scale. The use of power-law forcing is essential in most perturbative treatments because only the limit of long-range enough forc- ing is well-controlled perturbatively [36]. An extrapolation (depending on the dimension) is then required to recover the behavior corresponding to a forcing dominated by the integral scale, which constitutes an essential difficulty in most perturbative analyses. In contrast, we show that in the nonperturbative framework, this strategy is unnecessary. A fixed point that describes turbulence with integral-scale forcing is approached without the need of power-law forcing. In fact, in [86], both a power-law and a peaked component of the forcing are considered. Interestingly, the authors observe a transition in the $\epsilon$-dependence of observables at a threshold value of $\epsilon$, beyond which the power-law forcing plays a subdominant role compared to the localized forcing. A similar transition between a long-range (LR) regime with $\epsilon$-dependent exponent and a short-range (SR) regime with $\epsilon$-independent exponent, occurring at a critical value $\epsilon(d)$ of $\epsilon$, also exists in the KPZ model in the presence of both a microscopic delta-correlated noise (SR) and a power-law noise (LR). In this model, the transition from one regime to the other is naturally explained by the presence of two fixed points whose stability and basin of attraction depend on $\epsilon$ [82]. At large $\epsilon$ where the LR noise is relevant, the long-distance behavior of the model is governed by one fixed point (the LR fixed point), characterized by critical exponents depending on $\epsilon$. As $\epsilon$ is decreased, LR moves and eventually crosses the usual KPZ fixed point (the SR fixed point) at $\epsilon_{c}(d)$. Below $\epsilon_{c}(d)$, the stability of SR and LR are interchanged and SR becomes fully attractive: the LR power-law part of the noise no longer plays a role. We conjecture here that the same scenario occurs in the NS case: In the presence of both a power-law forcing and a forcing centered at the integral scale, two fixed points exist which collide and exchange their stability at $\epsilon=\epsilon_{c}(d)$ (with $\epsilon_{c}(d=3)=3 / 2$ ). Under this hypothesis, the explanation of the "saturation" of the exponent for $\epsilon>\epsilon_{c}(d)$ boils down to the change of stability of the two fixed points when they collide. An interesting outcome of our NPRG approach is to show that the fixed-point corresponding to a "physical" forcing applied at the integral scale only, can be described without the need to introduce a power-law component of the forcing.

Our analysis is based on the general strategy of the NPRG in its modern implementation 62], adapted for classical non-equilibrium systems [89, 90]. The NPRG formalism to study the NS equation is set up and presented in Sec. II] As common with field-theoretic methods, the symmetries play a crucial role. The accuracy of the results obtained with the NPRG approach depends on the order of the approximation implemented, and to preserve all the symmetries of the initial problem along the RG flow is of particular importance to ensure that it takes place in the appropriate functional (in fields, momenta and frequencies) space. The Navier-Stokes field theory admits, besides the well-known Galilean invariance and its time-gauged (also named time-dependent, or extended) version [12, 14, 91 94], another gauge symme- 
try, presented in Ref. [95]. Both these gauge symmetries are briefly reviewed in Sec. [II]

We then follow two complementary routes. The first one is closely related to the works of Refs. 79 83] on the KPZ equation, and also to [84, 86] in the NS context. An ansatz for the scale-dependent generating functional $\Gamma_{\kappa}$ of the (one-particle-irreducible) correlation and response functions is proposed and its evolution is followed between the Kolmogorov microscale and the macroscopic scale. The choice of the ansatz is strongly constrained by the gauge symmetries of the NS field theory, which hence play a fundamental role. We begin in Sec. $\mathrm{V}$ with building the appropriate ansatz (at Leading Order (LO) approximation), which exactly encodes these symmetries, and derive the corresponding NPRG flow equations. We show in Sec. VI that the RG flow is generically (without fine-tuning any parameter) attracted towards a fixed point, which corresponds to stationary fully developed turbulence generated by integral-scale forcing, both in $d=2$ and $d=3$.

The scaling properties of the turbulent steady state are analyzed within this approximation. Let us recall that, in $d=3$, Kolmogorov K41 theory predicts a $p^{-5 / 3}$ decay of the energy spectrum in the inertial range, associated with the direct cascade of energy. In $d=2$, part of the energy is transferred towards the large scale in the inverse cascade [6], with a $p^{-5 / 3}$ spectrum, whereas enstrophy flows towards the small scale in the direct cascade, yielding a steeper $p^{-3}$ energy spectrum, according to the Kraichnan-Batchelor (KB) theory [6, 7]. Within the LO approximation, we find that the energy spectrum (and the second-order structure function) computed at the fixed point follow $\mathrm{K} 41$ predictions in $d=3$ and $\mathrm{KB}$ ones in $d=2$. This was already observed by Tomassini in $d=3$ [84], and by the authors of [86] in the regime dominated by the localized forcing. This observation is compatible with experiments and numerical simulations, in so far as they find very small - if any - deviations from the dimensional scaling for these quantities [4]. One hence needs to very precisely study the large wave-number behavior to determine whether there exist such corrections. However, the LO approximation is not appropriate for this. Indeed, even though this approximation is well controlled and reliable for quantities defined at wave-numbers smaller than or comparable to the inverse integral scale, such as the existence of the fixed point, it is not justified at wave-numbers much larger than the inverse integral scale and its predictions in this regime should be taken with care.

In Sec. VII we undertake a second complementary strategy, specifically focusing on the large wave-number regime of the NPRG flow equations, and deeply rooted in the symmetries. This second approach has never been undertaken in previous works. We show that a set of exact and closed flow equations for the two-point functions can be derived in the large (compared to the running scale of the flow) wave-number regime, by using the Ward identities ensuing from the gauge symmetries of the theory. From these equations, we prove (without approximations) that the exact fixed point does not entail the usual scale invariance in the large wave-number regime, and we expound the mechanism of emergence of multiscaling within the NPRG framework. It originates in this formalism in a violation of the property of decoupling of the wave-number scales (the two fundamental UV and IR scales play a role all along the flow), which is not encountered in ordinary critical phenomena. The consequence is that the behavior at large-wave numbers of the correlation functions (the exponent of the power law) is not fixed in term of the scaling dimension of the velocity field and hence may deviate from dimensional predictions (K41 in $d=3$ and $\mathrm{KB}$ in $d=2$ ). The nondecoupling property is related to the absence of a regular limit when the integral scale (the typical length scale of energy injection) tends to infinity. It means that the salient scale for intermittency is the integral scale and not the UV one. The same observation underlies the OPE approaches in the perturbative context [19]. However, the precise link between the absence of operators of negative dimensions in the OPE and the non-decoupling property is not straightforward, and deserves further investigations. We emphasize that these exact equations in the large wave-number sector complement the flow equations obtained from the LO approximation, which is valid in the small wave-number regime. Their numerical solution, that we leave for a future publication, should allow us to obtain from first principles (although approximately) the intermittency exponents for the two-point functions.

\section{NPRG FORMALISM FOR NAVIER-STOKES TURBULENCE}

\section{A. Navier-Stokes field theory}

The NS equation (1) in the presence of the stochastic forcing $\vec{f}$ formally resembles a Langevin equation. One can resort to the standard Martin-Siggia-Rose-Janssende Dominicis procedure [96 98 to derive the associated field theory. Following Ref. 95], we introduce MartinSiggia-Rose response fields $\bar{v}_{\alpha}$ and $\bar{p}$ to enforce both the equation of motion (11) and the incompressibility constraint (2). Note that in this derivation, the pressure field is kept (instead of being eliminated as the solution, expressed in terms of the velocity and of the forcing, of a Poisson equation), because the pressure sector turns out to be very simple to handle since it is not renormalized [95]. Once the response fields are introduced, the stochastic forcing can be integrated out and one obtains the generating functional 95.

$$
\begin{aligned}
\mathcal{Z}[\vec{J}, \overline{\vec{J}}, K, \bar{K}] & =\int \mathcal{D} \vec{v} \mathcal{D} p \mathcal{D} \overline{\vec{v}} \mathcal{D} \bar{p} e^{-\left(\mathcal{S}_{0}[\vec{v}, \overline{\vec{v}}, p, \bar{p}]+\Delta \mathcal{S}_{0, L^{-1}}[\vec{v}, \bar{v}]\right)} \\
& \times e^{\int_{\mathbf{x}}\{\vec{J} \cdot \vec{v}+\overline{\bar{J}} \cdot \overline{\vec{v}}+K p+\bar{K} \bar{p}\}}
\end{aligned}
$$


where $\vec{J}, K, \overline{\vec{J}}$ and $\bar{K}$ are sources for the velocity, pressure and response fields [99], and where the NS action, splitted in a local and a nonlocal contribution for convenience, is given by

$$
\begin{aligned}
\mathcal{S}_{0}[\vec{v}, \overline{\vec{v}}, p, \bar{p}] & =\int_{\mathbf{x}}\left\{\bar{p}(\mathbf{x}) \partial_{\alpha} v_{\alpha}(\mathbf{x})+\bar{v}_{\alpha}(\mathbf{x})\left[\partial_{t} v_{\alpha}(\mathbf{x})\right.\right. \\
& \left.\left.-\nu \nabla^{2} v_{\alpha}(\mathbf{x})+v_{\beta}(\mathbf{x}) \partial_{\beta} v_{\alpha}(\mathbf{x})+\frac{1}{\rho} \partial_{\alpha} p(\mathbf{x})\right]\right\} \\
\Delta \mathcal{S}_{0, L^{-1}}[\vec{v}, \overline{\vec{v}}] & =-\int_{t, \vec{x}, \vec{x}^{\prime}} \bar{v}_{\alpha}(t, \vec{x}) N_{L^{-1}, \alpha \beta}\left(\left|\vec{x}-\vec{x}^{\prime}\right|\right) \bar{v}_{\beta}\left(t, \vec{x}^{\prime}\right) .
\end{aligned}
$$

where $\mathbf{x} \equiv(t, \vec{x})$ and $\int_{\mathbf{x}} \equiv \int d^{d} \vec{x} d t$. Let us now discuss the choice of the forcing profile. Without loss of generality, in order to preserve rotational invariance along the flow, it can be written in Fourier space as

$$
N_{\kappa, \alpha \beta}(\vec{q}) \equiv \delta_{\alpha \beta} N_{\kappa}(\vec{q})+q_{\alpha} q_{\beta} \hat{N}_{\kappa}(\vec{q})
$$

where the inverse integral scale $L^{-1}$ is denoted $\kappa$ in anticipation since it will be running in the following. The Fourier convention, used throughout this work, is

$$
\begin{aligned}
& f(\mathbf{q})=\int_{\mathbf{x}} f(\mathbf{x}) e^{-i \vec{q} \cdot \vec{x}+i \omega t} \\
& f(\mathbf{x})=\int_{\mathbf{q}} f(\mathbf{q}) e^{i \vec{q} \cdot \vec{x}-i \omega t}
\end{aligned}
$$

where $\mathbf{q} \equiv(\omega, \vec{q})$ and $\int_{\mathbf{q}} \equiv \int \frac{d^{d} \vec{q}}{(2 \pi)^{d}} \frac{d \omega}{2 \pi}$. In practice, due to the incompressibility condition, the term proportional to $q_{\alpha} q_{\beta}$ plays no role and can be omitted. We further parametrize the function $N_{\kappa}$ as

$$
N_{\kappa}(\vec{q})=D_{\kappa}(|\vec{q}| / \kappa)^{2} \hat{n}(|\vec{q}| / \kappa)
$$

where $D_{\kappa}$ is a scale-dependent coefficient, discussed in Sec. VD $N_{\kappa}(\vec{q})$ vanishes at $\vec{q}=0$ in order not to imprint a global motion to the fluid. The stirring force profile $\hat{n}$, peaked at the inverse integral scale $\kappa$, can be typically shaped as

$$
\hat{n}(x)=e^{-x^{2}} .
$$

Let us report that ten different forcing profiles have been studied in Ref. [84], which shows that the influence of the precise form of the stirring is negligible, or inexistent (as expected from universality) in the sense that the properties of the turbulent flow in the stationary regime do not change. We can hence restrict our analysis to the specific profile (9). It also corresponds to the local component of the forcing chosen in [86].

\section{B. NPRG formalism}

The general NPRG formalism for nonequilibrium systems in classical physics is presented in details in Refs.
[89, 90]. In the spirit of Wilson's RG ideas, it consists in building a sequence of scale-dependent effective models such that fluctuations are smoothly averaged as the (wave-number) scale $k$ is lowered from the microscopic UV scale $k=\eta^{-1}$ (inverse Kolmogorov scale), where no fluctuations are yet included, to the macroscopic IR scale $k=0$ (infinite volume), where they are all summed over 62, 100]. The procedure is formally the same as in equilibrium [62], but with the presence of response fields, and additional requirements stemming from Itō's discretization and causality issues 90, 101].

In this work, we identify the RG wave-number scale $k$ with the inverse of the integral scale $\kappa$, i.e. $k \equiv \kappa$. The integral scale is therefore running and eventually sent to infinity when $k \rightarrow 0$ (similarly to Refs. [84, 86]). For other purposes, the integral (injection) scale and the inverse volume scale can be kept independent, and the RG scale chosen as one of them while keeping the other fixed. For instance, the study of the RG flow at a fixed integral scale $L$ in the infinite volume limit (RG scale tends to zero) would be relevant to access the properties of the inverse cascade in bidimensional turbulence. This important issue will be investigated in a future work.

To achieve the separation of fluctuation modes within the NPRG procedure, a wave-number and scaledependent quadratic (regulator) term $\Delta \mathcal{S}_{\kappa}$ is added to the original action $\mathcal{S}_{0}+\Delta \mathcal{S}_{0, L^{-1}}$. On the one hand, we let the inverse integral scale run in the nonlocal quadratic term $\Delta \mathcal{S}_{0, L^{-1}} \rightarrow \Delta \mathcal{S}_{0, \kappa}$ (as in [84] and [86]). On the other hand, we include an additional scale dependent quadratic term to obtain the following regulator

$$
\begin{aligned}
\Delta \mathcal{S}_{\kappa}[\vec{v}, \overline{\vec{v}}] & =-\int_{t, \vec{x}, \vec{x}^{\prime}} \bar{v}_{\alpha}(t, \vec{x}) N_{\kappa, \alpha \beta}\left(\left|\vec{x}-\vec{x}^{\prime}\right|\right) \bar{v}_{\beta}\left(t, \vec{x}^{\prime}\right) \\
& +\int_{t, \vec{x}, \vec{x}^{\prime}} \bar{v}_{\alpha}(t, \vec{x}) R_{\kappa, \alpha \beta}\left(\left|\vec{x}-\vec{x}^{\prime}\right|\right) v_{\beta}\left(t, \vec{x}^{\prime}\right) .
\end{aligned}
$$

The additional $R_{\kappa}$ term, proportional to the velocity, can be interpreted as an Eckman friction term. Its presence is fundamental in $d=2$ to damp energy transfer towards larger and larger scales. It introduces an effective energy dissipation at the boundary of the effective volume $\kappa^{-d}$. Its effect is hence to suppress fluctuations with wavenumbers smaller than $\kappa$. Varying the scale $\kappa$ of $R_{\kappa}$ is conceptually equivalent to varying the volume of the system, it is hence analogous in a way to studying finite-size scaling. As previously, by using the incompressibility of the flow, the function $R_{\kappa}$ can be chosen diagonal. We write it in Fourier space as

$$
R_{\kappa, \alpha \beta}(\vec{q})=\delta_{\alpha \beta} R_{\kappa}(\vec{q})=\delta_{\alpha \beta} \nu_{\kappa} \vec{q}^{2} \hat{r}\left(q^{2} / \kappa^{2}\right)
$$

with $q=|\vec{q}|$ and where $\nu_{\kappa}$ is the scale-dependent viscosity, discussed in Sec. VD The cutoff function $\hat{r}(x)$ ensures the selection of fluctuation modes: $\hat{r}(x)$ is required to almost vanish for $x \gtrsim 1$ such that the fluctuation modes $v_{\alpha}(q \gtrsim \kappa)$ and $\bar{v}_{\alpha}(q \gtrsim \kappa)$ are unaffected by the $R_{\kappa}$ term in $\Delta \mathcal{S}_{\kappa}$, and to be large when $x \lesssim 1$ such that the other modes $\left(v_{\alpha}(q \lesssim \kappa)\right.$ and $\left.\bar{v}_{\alpha}(q \lesssim \kappa)\right)$ are 
essentially frozen. One can show that the form (10) of regulator term preserves all the symmetries and causality properties of the problem as done in a very similar case in [80]. We work here with the following cutoff function

$$
\hat{r}(x)=\frac{a}{e^{x}-1}
$$

where $a$ is a free parameter, which can be varied to assess the accuracy of the approximation scheme 102]. Let us emphasize that the addition of the regulator term $R_{\kappa}$ is essential to properly implement the RG procedure and to correctly regularize the flow, both in the UV and in the IR, as already realized in [86]. This constitutes a fundamental difference with the work of Ref. [84], where the term $R_{\kappa}$ is missing, and only the forcing term $N_{\kappa}$ acts to select the fluctuation modes. Although the procedure of [84] qualitatively leads to the correct behavior in $d=3$, it clearly prevents from studying the $d=2$ case because the flow equations are IR divergent in this dimension without the $R_{\kappa}$ term. Conversely, the NPRG flow equations derived in [86] and in the present work are properly regulated in any dimensions.

In the presence of the regulator term $\Delta \mathcal{S}_{\kappa}$, the generating functional (4) becomes scale dependent

$$
\begin{aligned}
\mathcal{Z}_{\kappa}[\vec{J}, \overline{\vec{J}}, K, \bar{K}] & =\int \mathcal{D} \vec{v} \mathcal{D} p \mathcal{D} \overline{\vec{v}} \mathcal{D} \bar{p} e^{-\left(\mathcal{S}_{0}[\vec{v}, \overline{\vec{v}}, p, \bar{p}]+\Delta \mathcal{S}_{\kappa}[\vec{v}, \overline{\vec{v}}]\right)} \\
& \times e^{\int_{\mathbf{x}}\{\vec{J} \cdot \vec{v}+\overline{\bar{J}} \cdot \overline{\vec{v}}+K p+\bar{K} \bar{p}\}} .
\end{aligned}
$$

Field expectation values in the presence of the external sources $\vec{J}, \overline{\vec{J}}, K$, and $\bar{K}$ are obtained as functional derivatives of $\mathcal{W}_{\kappa}=\log \mathcal{Z}_{\kappa}$ as

$$
u_{\alpha}(\mathbf{x})=\left\langle v_{\alpha}(\mathbf{x})\right\rangle=\frac{\delta \mathcal{W}_{\kappa}}{\delta J_{\alpha}(\mathbf{x})}, \bar{u}_{\alpha}(\mathbf{x})=\left\langle\bar{v}_{\alpha}(\mathbf{x})\right\rangle=\frac{\delta \mathcal{W}_{\kappa}}{\delta \bar{J}_{\alpha}(\mathbf{x})}
$$

and similarly for the pressure fields, for which for simplicity the same notation can be kept for the fields and their average values

$$
p(\mathbf{x}) \equiv\langle p(\mathbf{x})\rangle=\frac{\delta \mathcal{W}_{\kappa}}{\delta K(\mathbf{x})}, \bar{p}(\mathbf{x}) \equiv\langle\bar{p}(\mathbf{x})\rangle=\frac{\delta \mathcal{W}_{\kappa}}{\delta \bar{K}(\mathbf{x})} .
$$

The effective average action $\Gamma_{\kappa}[\vec{u}, \overline{\vec{u}}, p, \bar{p}]$ is defined as the Legendre transform of $\mathcal{W}_{\kappa}$ (up to terms proportional to $R_{\kappa, \alpha \beta}$ or $N_{\kappa, \alpha \beta}$ [ [62, 90]:

$$
\begin{aligned}
\Gamma_{\kappa}[\vec{u}, \overline{\vec{u}}, p, \bar{p}] & +\mathcal{W}_{\kappa}[\vec{J}, \overline{\vec{J}}, K, \bar{K}]=\int_{\vec{x}} j_{i} \varphi_{i} \\
& -\int_{t, \vec{x}, \vec{x}^{\prime}}\left\{\bar{u}_{\alpha} R_{\kappa, \alpha \beta} u_{\beta}-\bar{u}_{\alpha} N_{\kappa, \alpha \beta} \bar{u}_{\beta}\right\}
\end{aligned}
$$

where $\varphi_{i}, i=1, \ldots, 4$, stand for the fields $u_{\alpha}, \bar{u}_{\alpha}, p$ and $\bar{p}$, respectively, and $j_{i}$ for the sources $J_{\alpha}, \bar{J}_{\alpha}, K$ and $\bar{K}$, respectively. From $\Gamma_{\kappa}$, one can derive 2-point correlation and response functions, which can be gathered in a $4 \times 4$ matrix as

$$
\left[\Gamma_{\kappa}^{(2)}\right]_{i_{1} i_{2}}\left(\mathbf{x}_{1}, \mathbf{x}_{2},\left\{\varphi_{i}\right\}\right)=\frac{\delta^{2} \Gamma_{\kappa}\left[\left\{\varphi_{i}\right\}\right]}{\delta \varphi_{i_{1}}\left(\mathbf{x}_{1}\right) \delta \varphi_{i_{2}}\left(\mathbf{x}_{2}\right)}
$$

and more generally $n$-point correlation functions that are also written in a $4 \times 4$ matrix form as

$$
\Gamma_{\kappa, i_{3}, \ldots, i_{n}}^{(n)}\left(\mathbf{x}_{1}, \ldots, \mathbf{x}_{n},\left\{\varphi_{i}\right\}\right)=\frac{\delta^{n-2} \Gamma_{\kappa}^{(2)}\left(\mathbf{x}_{1}, \mathbf{x}_{2},\left\{\varphi_{i}\right\}\right)}{\delta \varphi_{i_{3}}\left(\mathbf{x}_{3}\right) \ldots \delta \varphi_{i_{n}}\left(\mathbf{x}_{n}\right)}
$$

The exact flow for $\Gamma_{\kappa}[\vec{u}, \overline{\vec{u}}, p, \bar{p}]$ is given by Wetterich equation, which reads in Fourier space [62, 103]

$$
\partial_{\kappa} \Gamma_{\kappa}=\frac{1}{2} \operatorname{Tr} \int_{\mathbf{q}} \partial_{\kappa} \mathcal{R}_{\kappa}(\mathbf{q}) \cdot G_{\kappa}(\mathbf{q})
$$

where $\mathcal{R}_{\kappa}(\mathbf{q})$ is the Fourier transform of the $4 \times 4$ matrix $\Delta \mathcal{S}_{\kappa}^{(2)}$. One can infer from definition (10) that its sole non-vanishing elements are $\left[\mathcal{R}_{\kappa}\right]_{22}=-2 N_{\kappa, \alpha \beta}$ and $\left[\mathcal{R}_{\kappa}\right]_{12}=\left[\mathcal{R}_{\kappa}\right]_{21}=R_{\kappa, \alpha \beta}$. The matrix

$$
G_{\kappa} \equiv\left[\Gamma_{\kappa}^{(2)}+\mathcal{R}_{\kappa}\right]^{-1}
$$

is the full, that is, field-dependent, renormalized at scale $\kappa$ propagator of the theory. When the RG scale $\kappa$ is lowered from the UV scale $\eta^{-1}$ to zero, $\Gamma_{\kappa}$ interpolates between the microscopic model $\Gamma_{\kappa=\eta^{-1}}=\mathcal{S}_{0}$ and the full effective action $\Gamma_{\kappa=0}$ that encompasses all the macroscopic properties of the system (for a detailed discussion in nonequilibrium processes, see Ref. [90]). Differentiating Eq. (17) twice with respect to the fields and evaluating the resulting identity in a uniform and stationary field configuration $\varphi_{i}(\mathbf{x})=\varphi_{i}$ (since the model is analyzed in its long time and large distance regime where it is translationally invariant in space and time) one obtains the flow equation for the 2-point functions:

$$
\begin{aligned}
& \partial_{\kappa} \Gamma_{\kappa, i j}^{(2)}(\mathbf{p})=\operatorname{Tr} \int_{\mathbf{q}} \partial_{\kappa} \mathcal{R}_{\kappa}(\mathbf{q}) \cdot G_{\kappa}(\mathbf{q}) \cdot\left(-\frac{1}{2} \Gamma_{\kappa, i j}^{(4)}(\mathbf{p},-\mathbf{p}, \mathbf{q})\right. \\
& \left.+\Gamma_{\kappa, i}^{(3)}(\mathbf{p}, \mathbf{q}) \cdot G_{\kappa}(\mathbf{p}+\mathbf{q}) \cdot \Gamma_{\kappa, j}^{(3)}(-\mathbf{p}, \mathbf{p}+\mathbf{q})\right) \cdot G_{\kappa}(\mathbf{q})
\end{aligned}
$$

where the background field $\varphi_{i}$ dependencies are implicit, as well as the last arguments of the $\Gamma_{\kappa}^{(n)}$ which are determined by frequency and wave-vector conservation [90].

Of course Eq. (17) cannot be solved exactly and one has to resort to an appropriate approximation scheme, adapted to the physics of the model under study, and in particular to its symmetries, that are reviewed in the next section.

\section{SYMMETRIES AND RELATED WARD IDENTITIES}

In this section, we briefly review the three gauge symmetries of the NS action expounded in Ref. [95] and the ensuing non-renormalization theorems and general Ward identities derived in this Reference. 


\section{A. Symmetries}

The NS action $\mathcal{S} \equiv \mathcal{S}_{0}+\Delta \mathcal{S}_{\kappa}$ given by (5) admit three gauge symmetries:

- (i) invariance under gauged shifts of the pressure fields,

- (ii) time-gauged Galilean symmetry,

- (iii) invariance under a time-gauged shift of the response fields.

The symmetry (i) is the invariance of $\mathcal{S}$ under the local shifts $p(t, \vec{x}) \rightarrow p(t, \vec{x})+\epsilon(t, \vec{x})$ or $\bar{p}(t, \vec{x}) \rightarrow \bar{p}(t, \vec{x})+$ $\bar{\epsilon}(t, \vec{x})$, which implies that the equations of motion for $p$ and $\bar{p}$ are exactly given by the minimization of the bare action $\mathcal{S}_{0}$. The infinitesimal time-gauged Galilean symmetry (ii), also referred to as time-dependent [12, 92, 93], or extended [94, 104] Galilean symmetry, consists in the following field transformation

$$
\begin{aligned}
\delta v_{\alpha}(\mathbf{x}) & =-\dot{\epsilon}_{\alpha}(t)+\epsilon_{\beta}(t) \partial_{\beta} v_{\alpha}(\mathbf{x}) \\
\delta \bar{v}_{\alpha}(\mathbf{x}) & =\epsilon_{\beta}(t) \partial_{\beta} \bar{v}_{\alpha}(\mathbf{x}) \\
\delta p(\mathbf{x}) & =\epsilon_{\beta}(t) \partial_{\beta} p(\mathbf{x}) \\
\delta \bar{p}(\mathbf{x}) & =\epsilon_{\beta}(t) \partial_{\beta} \bar{p}(\mathbf{x})
\end{aligned}
$$

where $\dot{\epsilon}_{\alpha}=\partial_{t} \epsilon_{\alpha}$. When $\vec{\epsilon}(t) \equiv \vec{\epsilon}$ is an arbitrary constant vector, the transformation corresponds to a translation in space, and when $\vec{\epsilon}(t) \equiv \vec{\epsilon} t$ it corresponds to the usual (non-gauged) Galilean transformation. Lastly, the infinitesimal time-gauged shift symmetry (iii) consists in the field transformation

$$
\begin{aligned}
\delta \bar{v}_{\alpha}(\mathbf{x}) & =\bar{\epsilon}_{\alpha}(t) \\
\delta \bar{p}(\mathbf{x}) & =v_{\beta}(\mathbf{x}) \bar{\epsilon}_{\beta}(t) .
\end{aligned}
$$

For each of these transformations, the different terms of the NS action $\mathcal{S}$ are either invariant, or have a linear variation in the fields. The corresponding non-invariant terms play the role of gauge fixing controllable terms. By explicitly performing these transformations as changes of variables in the functional integral (13), and exploiting that they must leave it unaltered, one deduces general Ward identities. These identities are derived in Ref. 95] for the original (microscopic) NS field theory. The procedure can be directly transposed to the scale dependent effective average action $\Gamma_{\kappa}$, by simply replacing the original quantities $(e . g . \mathcal{Z}, \Gamma)$ by the running ones $\left(\right.$ e.g. $\left.\mathcal{Z}_{\kappa}, \Gamma_{\kappa}\right)$. Note also that since $\Delta \mathcal{S}_{\kappa}$ is substracted in the (modified) definition of the Legendre transform (14) in contrast to Ref. [95], the corresponding terms (proportional to $N_{\kappa, \alpha \beta}$ or $R_{\kappa, \alpha \beta}$ ) are removed from the Ward identities. These identities are recapitulated below.

\section{B. Ward identities}

The Ward identities ensuing from the gauged-shift symmetries (i) simply read

$$
\frac{\delta \Gamma_{\kappa}}{\delta p(\mathbf{x})}=\frac{\delta \mathcal{S}_{0}}{\delta p(\mathbf{x})} \quad \text { and } \quad \frac{\delta \Gamma_{\kappa}}{\delta \bar{p}(\mathbf{x})}=\frac{\delta \mathcal{S}_{0}}{\delta \bar{p}(\mathbf{x})}
$$

which means that the dependence in $p(\mathbf{x})$ and $\bar{p}(\mathbf{x})$ of both the effective action $\Gamma_{\kappa}$ and the bare one $\mathcal{S}_{0}$ are identical. One thus concludes that the whole pressure sector is not renormalized. Of course, connected correlation functions of the pressure do have corrections coming from fluctuations. This is a simplifying feature of the 1PI effective action, which keeps exactly the same pressure dependence as the bare action.

The NS action is invariant under the time-gauged Galilean transformation (20), but for the term proportional to the Lagrangian time derivative $D_{t} v_{\alpha}(\mathbf{x}) \equiv$ $\partial_{t} v_{\alpha}(\mathbf{x})+v_{\beta}(\mathbf{x}) \partial_{\beta} v_{\alpha}(\mathbf{x})$, which variation is

$$
\delta \int_{\mathbf{x}} \bar{v}_{\alpha}(\mathbf{x}) D_{t} v_{\alpha}(\mathbf{x}) \equiv \delta \mathcal{S}=-\int_{\mathbf{x}} \ddot{\epsilon}_{\alpha}(t) \bar{v}_{\alpha}(\mathbf{x}) .
$$

Hence, requiring that the change of variables (20) leaves the functional integral (13) unaltered, one obtains the following Ward identity

$$
\begin{aligned}
& \int_{\vec{x}}\left\{\left(\delta_{\alpha \beta} \partial_{t}+\partial_{\beta} u_{\alpha}(\mathbf{x})\right) \frac{\delta \Gamma_{\kappa}}{\delta u_{\alpha}(\mathbf{x})}+\partial_{\beta} \bar{u}_{\alpha}(\mathbf{x}) \frac{\delta \Gamma_{\kappa}}{\delta \bar{u}_{\alpha}(\mathbf{x})}\right. \\
& \left.+\partial_{\beta} p(\mathbf{x}) \frac{\delta \Gamma_{\kappa}}{\delta p(\mathbf{x})}+\partial_{\beta} \bar{p}(\mathbf{x}) \frac{\delta \Gamma_{\kappa}}{\delta \bar{p}(\mathbf{x})}\right\}=-\int_{\vec{x}} \partial_{t}^{2} \bar{u}_{\beta}(\mathbf{x}),
\end{aligned}
$$

which implies that the variations of both the effective action and the bare one under time-gauged Galilean transformations are identical. This entails that, apart from the term $\int_{\mathbf{x}} \bar{u}_{\alpha}(\mathbf{x}) D_{t} u_{\alpha}(\mathbf{x})$ which is not renormalized and remains equal to its bare expression, $\Gamma_{\kappa}$ is invariant under these transformations.

As for the time-gauged shift symmetry (iii), the variation of the NS action under (21) is

$$
\delta \mathcal{S}=-\int_{\mathbf{x}} \dot{\bar{\epsilon}}_{\beta}(t) v_{\beta}(\mathbf{x}),
$$

and the related Ward identity reads

$$
\int_{\vec{x}}\left\{\frac{\delta \Gamma_{\kappa}}{\delta \bar{u}_{\alpha}(\mathbf{x})}+u_{\alpha}(\mathbf{x}) \frac{\delta \Gamma_{\kappa}}{\delta \bar{p}(\mathbf{x})}\right\}=\int_{\vec{x}} \partial_{t} u_{\alpha}(\mathbf{x}),
$$

meaning that, apart from the term $\int_{\mathbf{x}} \bar{u}_{\alpha} \partial_{t} u_{\alpha}$ which is not renormalized, the effective action $\Gamma_{\kappa}$ is invariant under time-gauged shift transformations.

Furthermore, as shown in Ref. [95], a functional Ward identity associated with a fully gauged (both in time and space) version of the shift symmetry (21) can be obtained in the presence of a local source term bilinear in the velocity field. This functional identity entails an infinite 
set of exact and local relations between correlation functions, which includes in particular the Kármán-Howarth relation [105]. From this fundamental relation, the exact Kolmogorov law for the third-order structure function in $d=3$ can be derived, assuming the existence of a dissipative anomaly (finite mean dissipation rate in the inviscid limit). The gauged shift symmetry hence plays a crucial role since it directly roots in symmetries the four-fifth law. From the functional Ward idendity can also be deduced another exact identity for a fourth-order pressure-velocity correlation function recently derived in Ref. [106], and further generalized in [95].

\section{General structure of the effective action $\Gamma_{\kappa}$}

One can infer from the previous Ward identities the general form of the effective action $\Gamma_{\kappa}$ :

$$
\begin{aligned}
\Gamma_{\kappa}[\vec{u}, \overline{\vec{u}}, p, \bar{p}] & =\int_{\mathbf{x}}\left\{\bar{u}_{\alpha}\left(\partial_{t} u_{\alpha}+\lambda u_{\beta} \partial_{\beta} u_{\alpha}+\frac{\partial_{\alpha} p}{\rho}\right)\right. \\
& \left.+\bar{p} \partial_{\alpha} u_{\alpha}\right\}+\tilde{\Gamma}_{\kappa}[\vec{u}, \overline{\vec{u}}]
\end{aligned}
$$

where the explicit terms are not renormalized and thus keep their bare forms, and the functional $\tilde{\Gamma}_{\kappa}$ is invariant under time-gauged Galilean and shift transformations. The coefficient $\lambda$ is introduced in front of the nonlinear term for later power counting purposes. Of course, $\lambda$ can always be set equal to one in appropriate units. Note that including this coefficient in the original NS action (5) induces some slight modifications of the related Ward identities by trivial factors $\lambda$. Yet, it still leads to the general form (27) of the effective action where $\lambda$ is not renormalized.

\section{CONSERVATION LAWS AND ENERGY SPECTRUM}

In this section, we study the different contributions to the energy and to the enstrophy and we show that the conservation of energy in $d=3$, and of both energy and enstrophy in $d=2$, yields constraints which fix the values of the anomalous dimensions. We also derive the general expressions for the energy spectrum and the second order structure function. These observables are explicitly computed in Sec. VI at LO approximation.

All these quantities can be expressed in terms of connected two-point correlation and response functions: $G_{\alpha \beta}^{u u}=\left\langle v_{\alpha} v_{\beta}\right\rangle_{c}$ and $G_{\alpha \beta}^{u \bar{u}}=\left\langle v_{\alpha} \bar{v}_{\beta}\right\rangle_{c}$. These functions are elements of the propagator matrix $G_{\kappa}$, which is defined as the inverse of the matrix $\Gamma_{\kappa}^{(2)}$. The general structure of the propagator matrix $G_{\kappa}$ is determined in Appendix A, its components in the velocity sector are reported below Eq. (30).

Let us clarify notation. Because of rotational and parity invariance, any generic two-(space)index function (in
Fourier space) $F_{\alpha \beta}(\omega, \vec{p})$ can be decomposed into a longitudinal and a transverse part

$$
F_{\alpha \beta}(\omega, \vec{p})=P_{\alpha \beta}^{\perp}(\vec{p}) F_{\perp}\left(\omega, \vec{p}^{2}\right)+P_{\alpha \beta}^{\|}(\vec{p}) F_{\|}\left(\omega, \vec{p}^{2}\right)
$$

where the transverse and longitudinal projectors are defined by

$$
P_{\alpha \beta}^{\perp}(\vec{p})=\delta_{\alpha \beta}-\frac{p_{\alpha} p_{\beta}}{\vec{p}^{2}}, \quad \text { and } \quad P_{\alpha \beta}^{\|}(\vec{p})=\frac{p_{\alpha} p_{\beta}}{\vec{p}^{2}} .
$$

As shown in Appendix A, the incompressibility condition entails that the components of the propagator in the velocity sector are purely transverse (that is, all the longitudinal parts vanish) and are given by

$$
\begin{aligned}
G_{\alpha \beta}^{\bar{u} \bar{u}}(\omega, \vec{q}) & =0 \\
G_{\alpha \beta}^{u \bar{u}}(\omega, \vec{q}) & =P_{\alpha \beta}^{\perp}(\vec{q}) \frac{1}{\Gamma_{\perp}^{(1,1)}(-\omega, \vec{q})+R_{\kappa}(\vec{q})} \\
G_{\alpha \beta}^{u u}(\omega, \vec{q}) & =-P_{\alpha \beta}^{\perp}(\vec{q}) \frac{\Gamma_{\perp}^{(0,2)}(\omega, \vec{q})-2 N_{\kappa}(\vec{q})}{\left|\Gamma_{\perp}^{(1,1)}(\omega, \vec{q})+R_{\kappa}(\vec{q})\right|^{2}} .
\end{aligned}
$$

For the following discussion, we also need to introduce renormalized and dimensionless quantities, denoted with a hat symbol. The wave-vectors and frequencies are respectively measured in units of $\kappa$ and $\kappa^{2} \nu_{\kappa}$, where $\nu_{\kappa}$ is the running viscosity. We thus define e.g. $\hat{p}=p / \kappa$ and $\hat{\omega}=\omega /\left(\kappa^{2} \nu_{\kappa}\right)$. Let us consider the expression (10) of the term $\Delta \mathcal{S}_{\kappa}$. The dimension of the cutoff term $R_{\kappa, \alpha \beta}$ is given by

$$
\left[\int_{\vec{x}^{\prime}} R_{\kappa, \alpha \beta}\left(\vec{x}-\vec{x}^{\prime}\right)\right]=\left[\kappa^{2} \nu_{\kappa}\right]
$$

and the dimension of the forcing term $N_{\kappa, \alpha \beta}$ can be inferred from definition (8)

$$
\left[\int_{\vec{x}^{\prime}} N_{\kappa, \alpha \beta}\left(\vec{x}-\vec{x}^{\prime}\right)\right]=\left[D_{\kappa}\right] .
$$

We associate two running anomalous dimensions $\eta_{\kappa}^{\nu}$ and $\eta_{\kappa}^{D}$ with these running coefficients as

$$
\eta_{\kappa}^{\nu}=-\partial_{s} \ln \nu_{\kappa} \quad \text { and } \quad \eta_{\kappa}^{D}=-\partial_{s} \ln D_{\kappa}
$$

where $\partial_{s} \equiv \kappa \partial_{\kappa}$. According to Eqs. (31) and (32), and since $\Gamma_{\kappa}$ and $\Delta \mathcal{S}_{\kappa}$ have the same dimension, one deduces from Eq. (27) the dimensions of the fields: $[u]=\left[\kappa^{d-2} D_{\kappa} \nu_{\kappa}^{-1}\right]^{1 / 2}$ and $[\bar{u}]=\left[\kappa^{d+2} \nu_{\kappa} D_{\kappa}^{-1}\right]^{1 / 2}$. We also introduce the dimensionless coupling $\hat{\lambda}_{\kappa}$ as

$$
\lambda=\left(\kappa^{-d+4} \nu_{\kappa}^{3} D_{\kappa}^{-1}\right)^{1 / 2} \hat{\lambda}_{\kappa}
$$

Since $\lambda$ is not renormalized, the flow equation for $\hat{\lambda}_{\kappa}$ is purely dimensional and reads

$$
\partial_{s} \hat{\lambda}_{\kappa}=\hat{\lambda}_{\kappa}\left(\frac{d}{2}-2+\frac{3}{2} \eta_{\kappa}^{\nu}-\frac{1}{2} \eta_{\kappa}^{D}\right)
$$


Of course, this is a direct consequence of Galilean symmetry and this equation (or similar forms) has already been obtained in other RG approaches [19, 84]. It follows from this equation that any non-Gaussian fixed point, with $\hat{\lambda}_{\kappa} \neq 0$, is characterized by a single independent anomalous dimension, for instance $\eta_{*}^{D}$, with

$$
\eta_{*}^{\nu}=4 / 3+\left(\eta_{*}^{D}-d\right) / 3 .
$$

We now show that the value of $\eta_{\kappa}^{D}$ is actually fixed by conservation laws.

\section{A. Energy conservation}

As recalled in the introduction, energy must be permanently injected at the integral scale $\kappa^{-1}$ to maintain a turbulent flow since the NS equation is dissipative. Hence, reaching the stationary regime of fully developed turbulence requires that the mean rate of injected power by unit mass $\left\langle\epsilon_{\mathrm{inj}}\right\rangle$ of the fluid compensates the mean rate of dissipated power by unit mass. Of course, if the full dynamics was studied starting from well-defined initial conditions, the conservation of energy would be automatically satisfied at all times and in particular in the long-time limit where the system reaches stationarity. Here, we directly study the steady state (assuming translational invariance in time). Indeed, the inclusion of the transcient regime is challenging and would be interesting to address in the future. Hence, we need to impose stationarity as an external constraint, in the form of a balance between the injected and dissipated energy. This was already realized in [84]. In fact, this is reminiscent of the additional assumption (existence of a dissipative anomaly) needed to derive the four-fifth law from the Kármán-Howarth relation.

There are two sources of energy dissipation in the NPRG setting, the dissipation by molecular viscosity at the microscopic Kolmogorov scale $\eta$ and the effective dissipation at the boundaries induced by the cutoff term proportional to $R_{\kappa}$ in (10). Denoting $\left\langle\epsilon_{\text {dis }}^{1 / \eta}\right\rangle$ and $\left\langle\epsilon_{\text {dis }}^{\kappa}\right\rangle$ their respective rate per unit mass, the energy balance equation in the steady state reads

$$
\left\langle\epsilon_{\mathrm{inj}}\right\rangle=\left\langle\epsilon_{\text {dis }}^{1 / \eta}\right\rangle+\left\langle\epsilon_{\text {dis }}^{\kappa}\right\rangle .
$$

Let us express these different contributions. As explained in Appendix C, the average injected power per unit mass can be expressed as

$$
\begin{aligned}
\left\langle\epsilon_{\mathrm{inj}}\right\rangle & =\left\langle f_{\alpha}(t, \vec{x}) v_{\alpha}(t, \vec{x})\right\rangle \\
& =\lim _{\delta t \rightarrow 0^{+}} \int_{\vec{x}^{\prime}} N_{\kappa, \alpha \beta}\left(\left|\vec{x}-\vec{x}^{\prime}\right|\right) G_{\alpha \beta}^{u \bar{u}}\left(t+\delta t, \vec{x} ; t, \vec{x}^{\prime}\right) \\
& =(d-1) D_{\kappa} \kappa^{d} \lim _{\delta t \rightarrow 0^{+}} \int_{\hat{\omega}, \hat{\vec{q}}} \hat{N}(\hat{\vec{q}}) e^{-i \hat{\omega} \hat{\delta t}} \hat{G}_{\perp}^{u \bar{u}}(\hat{\omega}, \hat{\vec{q}}) .
\end{aligned}
$$

The average effective dissipated power at the scale $\kappa$ can be written as

$$
\begin{aligned}
\left\langle\epsilon_{\mathrm{dis}}^{\kappa}\right\rangle & =\left\langle\int_{\vec{x}^{\prime}} v_{\alpha}(t, \vec{x}) R_{\kappa, \alpha \beta}\left(\left|\vec{x}-\vec{x}^{\prime}\right|\right) v_{\beta}\left(t, \vec{x}^{\prime}\right)\right\rangle \\
& =\int_{\omega, \vec{q}} R_{\kappa, \alpha \beta}(\vec{q}) G_{\alpha \beta}^{u u}(\omega, \vec{q}) \\
& =(d-1) D_{\kappa} \kappa^{d} \int_{\hat{\omega}, \hat{\vec{q}}} \hat{q}^{2} \hat{r}(\hat{\vec{q}}) \hat{G}_{\perp}^{u u}(\hat{\omega}, \hat{\vec{q}}) .
\end{aligned}
$$

Let us emphasize that, in the present analysis, once the scale $\kappa$ of $R_{\kappa}$ and the scale $k$ of $N_{k}$ have been equated, the effective energy dissipation (at the volume scale) and the energy injection (at the integral one) scale in the same way, as $D_{\kappa} \kappa^{d}$. The average dissipated power at the Kolmogorov scale per unit mass is expressed as

$$
\begin{aligned}
\left\langle\epsilon_{\mathrm{dis}}^{1 / \eta}\right\rangle & =\left\langle\nu \partial_{j} v_{i}(t, \vec{x}) \partial_{j} v_{i}(t, \vec{x})\right\rangle \\
& =\nu(d-1) \int_{\omega, \vec{q}} q^{2} G_{\perp}^{u u}(\omega, \vec{q}) .
\end{aligned}
$$

The behavior of this integral can be analyzed using the canonical behavior of $G_{\perp}^{u u}$ (intermittency corrections, if any, can be neglected in this argument, see Sec. VI). This canonical behavior can be deduced assuming (standard) scale invariance, see Eq. (94) and Sec. VIIB and is given in the inertial regime by

$$
G_{\perp}^{u u}(\omega, \vec{q})=q^{-\eta^{D}-4+2 \eta^{\nu}} g\left(\omega / q^{2-\eta^{\nu}}\right),
$$

where $g$ is a scaling function. One then obtains

$$
\begin{aligned}
\left\langle\epsilon_{\mathrm{dis}}^{1 / \eta}\right\rangle & =\frac{\nu(d-1)}{2^{d-1} \pi^{d / 2} \Gamma(d / 2)} \int_{0}^{1 / \eta} d q q^{\frac{2}{3}\left(d-\eta^{D}\right)+\frac{1}{3}} \\
& \times \int_{0}^{\infty} \frac{d x}{\pi} g(x)
\end{aligned}
$$

where Eq. (36) is used. If $\eta^{D} \lesssim d+2$, the integral on $q$ is UV divergent when $\eta \rightarrow 0$, which means that it is dominated by the UV scale $\eta^{-1}$. It follows that if $\eta^{D} \lesssim d+2$, $\left\langle\epsilon_{\text {dis }}^{1 / \eta}\right\rangle$ is independent of $\kappa$, and remains finite in the RG (infinite volume) limit $\kappa \rightarrow 0$. Hence, one deduces that to satisfy the energy budget equation (37), the average injected power $\left\langle\epsilon_{\mathrm{inj}}\right\rangle$ given by Eq. (38) minus the average effective dissipated power $\left\langle\epsilon_{\text {dis }}^{\kappa}\right\rangle$ given by Eq. (39) should also remain finite in the limit $\kappa \rightarrow 0$. Accordingly, either the two terms are both finite, or if one of them diverges in this limit, the other term must also diverge to compensate it. This implies $\kappa^{d} D_{\kappa} \geq \mathcal{O}(1)$ (as the integrals in (38) and (39) are finite), that is $\eta^{D} \geq d$.

\section{B. Case $d=3$}

In $d=3$, the energy is expected to be dominantly dissipated at the microscopic Kolmogorov scale $\eta$. This corresponds to the case $\eta^{D} \lesssim d+2$, with an average rate 
of dissipated power $\left\langle\epsilon_{\mathrm{dis}}^{1 / \eta}\right\rangle$ independent of $\kappa$. In the limit $\kappa \rightarrow 0$, the effective dissipation at the volume scale (fixed by $R_{\kappa}$ ) and the injected energy are of the same order $\left(\propto \kappa^{d} D_{\kappa}\right)$. However, as explained before, the integral scale and the volume scale could be kept independent. In $d=3$, the volume scale could be safely removed by sending $R_{\kappa} \rightarrow 0$ while keeping $N_{\kappa} \neq 0$ (as in Ref. 84]) and the flow equations would remain well-behaved. Otherwise stated, by taking the volume scale much larger than the integral scale, the dissipated energy at the volume scale can be rendered negligible compared to the injected energy in $d=3$. Accordingly, the injected energy cannot be compensated by the energy dissipated at the volume scale alone. This implies that it must behave as the energy dissipated at the Kolmogorov scale. As a consequence, the three terms in (37) must scale identically when $\kappa \rightarrow 0$ (that is, they must be independent of $\kappa$ when $\kappa \rightarrow 0)$.

We choose to impose this condition all along the RG flow (as should be done without the regulator $R_{\kappa}$ ). 107]. To keep a constant injected power while taking the RG (infinite volume) limit $\kappa \rightarrow 0$ thus requires to fix $\eta_{\kappa}^{D}=d$ for all $\kappa$, which means that $N_{\kappa}(\vec{q})$ must scale as $\kappa^{-d}$. This yields in $d=3$ that at the fixed point, $\eta_{*}^{\nu}=4 / 3$ according to Eq. (36). Hence the values of the two running anomalous dimensions at the fixed point are determined. For simplicity, since we are merely interested in the fixed point properties, we fix

$$
\eta_{\kappa}^{D}=3 \quad \text { and } \quad \eta_{\kappa}^{\nu}=4 / 3 \quad \text { in } \quad d=3
$$

for all $\kappa$, which has no influence on the fixed point properties.

\section{Case $d=2$}

In $d=2$, the situation is different. The energy is transferred both towards the small scales (direct cascade) and the large scales (inverse cascade) and is thus dissipated both at the microscopic Kolmogorov scale and at the boundaries of the system. This is manifest on the NPRG equations (67/68) that are no longer regular when sending $R_{\kappa} \rightarrow 0$ while keeping $N_{\kappa} \neq 0$. Accordingly, the argument used in $d=3$ (exploiting that the dissipation at the volume scale can be rendered negligible) is no longer valid in $d=2$ and only the previously shown inequality $\eta^{D} \geq d$ holds (one cannot conclude that $\eta_{\kappa}^{D}=d$ in this dimension). In order to fix $\eta^{D}$ for $d=2$, another conservation law is necessary.

One can exploit the enstrophy conservation to fix $\eta^{D}$ in a similar way that the conservation of energy was used in the $d=3$ case. The enstrophy flux is towards the small scale, in the direct cascade, and the dissipation of vorticity is dominated by the microscopic scale, and is thus independent of $\kappa$. The vorticity is defined as $\vec{\omega}(t, \vec{x})=\vec{\nabla} \times \vec{v}(t, \vec{x})$ which reduces in $d=2$ to the (pseudo-)scalar $\omega(t, \vec{x})=\epsilon_{i j} \partial_{i} v_{j}(t, \vec{x})$. The mean dissi- pation rate of vorticity can be expressed as [108]

$$
\begin{aligned}
\left\langle\omega_{\mathrm{dis}}\right\rangle & =\left\langle\nu(\vec{\nabla} \omega(t, \vec{x}))^{2}\right\rangle \\
& =\nu \int_{\omega, \vec{q}} q^{4} G_{\perp}^{u u}(\omega, \vec{q}) .
\end{aligned}
$$

Using the canonical behavior (41) of $G_{\perp}^{u u}$, one can indeed check that for $\eta_{D}<7$ in $d=2$ the integral on $q$ is UV divergent without a cutoff, and is hence dominated by the UV scale, and thus independent of $\kappa$. The conservation of enstrophy then requires that the mean injection rate of vorticity should also be independent of $\kappa$. This rate can be expressed as 108

$$
\begin{aligned}
\left\langle\omega_{\text {inj }}\right\rangle & =\langle(\vec{\nabla} \times \vec{f})(t, \vec{x}) \cdot \vec{\omega}(t, \vec{x})\rangle \\
& =\lim _{\delta t \rightarrow 0^{+}} \int_{\omega, \vec{q}} q^{2} N(\vec{q}) G_{\perp}^{u \bar{u}}(\omega, \vec{q}) e^{-i \omega \delta t} \\
& =D_{\kappa} \kappa^{4} \lim _{\delta t \rightarrow 0^{+}} \int_{\hat{\omega}, \hat{q}} \hat{q}^{2} \hat{N}(\vec{q}) \hat{G}_{\perp}^{u \bar{u}}(\hat{\omega}, \hat{\vec{q}}) e^{-i \hat{\omega} \hat{\delta} t} .
\end{aligned}
$$

One hence concludes that to keep a constant rate of injection of vorticity in the RG limit $\kappa \rightarrow 0$ requires to fix $\eta_{\kappa}^{D}=4$ for all $\kappa$. This identity satisfies the constraint $\eta_{\kappa}^{D} \geq d$ stemming from the energy conservation. Thus both enstrophy and energy are conserved in $d=2$. 109

This choice yields at the fixed point $\eta_{*}^{\nu}=2$, according to Eq. (36). The values of the two running anomalous dimensions at the fixed point are also determined in $d=$ 2 . As in $d=3$, and since we only consider the stationary regime, we simply fix these values all along the flow

$$
\eta_{\kappa}^{D}=4 \quad \text { and } \quad \eta_{\kappa}^{\nu}=2 \quad \text { in } \quad d=2 .
$$

\section{Energy spectrum}

The energy spectrum in dimension $d$ is usually defined as [4]

$$
E^{(d)}(\vec{p})=\frac{2 \pi^{d / 2}}{\Gamma(d / 2)} p^{d-1} \mathcal{E}^{(d)}(\vec{p})
$$

where $\mathcal{E}^{(d)}(\vec{p})$ is the Fourier transform of the equal-time velocity-velocity correlation function $\langle\vec{v}(t, \vec{x}) \cdot \vec{v}(t, \overrightarrow{0})\rangle$. The velocity-velocity correlation function (at arbitrary times) can be expressed as the inverse Fourier transform of $G_{\alpha \beta}^{u u}(\omega, \vec{q})$, that is

$$
\begin{aligned}
& \left\langle v_{\alpha}(t, \vec{x}) v_{\beta}(0, \overrightarrow{0})\right\rangle=\int_{\mathbf{q}} e^{-i(\omega t-\vec{q} \cdot \vec{x})} G_{\alpha \beta}^{u u}(\omega, \vec{q}) \\
& =-\int_{\mathbf{q}} e^{-i(\omega t-\vec{q} \cdot \vec{x})} P_{\alpha \beta}^{\perp}(\vec{q}) \frac{\Gamma_{\perp}^{(0,2)}(\omega, \vec{q})-2 N_{\kappa}(\vec{q})}{\left|\Gamma_{\perp}^{(1,1)}(\omega, \vec{q})+R_{\kappa}(\vec{q})\right|^{2}} .
\end{aligned}
$$


One hence obtains

$$
\begin{aligned}
\mathcal{E}^{(d)}(\vec{p}) & =\int d^{d} \vec{x} e^{-i \vec{p} \cdot \vec{x}}\left\langle v_{\alpha}(t, \vec{x}) v_{\alpha}(t, \overrightarrow{0})\right\rangle \\
& =-(d-1) \int_{0}^{\infty} \frac{d \omega}{\pi} \frac{\Gamma_{\perp}^{(0,2)}(\omega, \vec{p})-2 N_{\kappa}(\vec{p})}{\left|\Gamma_{\perp}^{(1,1)}(\omega, \vec{p})+R_{\kappa}(\vec{p})\right|^{2}} .
\end{aligned}
$$

We focus on the inertial regime where $|\vec{p}| \gg \kappa$, both in $d=2$ and $d=3$. In this limit the functions $N_{\kappa}(\vec{p})$ and $R_{\kappa}(\vec{p})$ tend to zero rapidly yielding

$$
\mathcal{E}^{(d)}(\vec{p}) \stackrel{|\vec{p}| \gg \kappa}{\simeq}-(d-1) \int_{0}^{\infty} \frac{d \omega}{\pi} \frac{\Gamma_{\perp}^{(0,2)}(\omega, \vec{p})}{\left|\Gamma_{\perp}^{(1,1)}(\omega, \vec{p})\right|^{2}}
$$

\section{E. Second-order structure function}

The longitudinal structure function of order $n$ is defined as the average of the $n^{\text {th }}$ power of the equal time longitudinal velocity increment

$$
S^{(n)}(\ell)=\left\langle[(\vec{v}(t, \vec{\ell})-\vec{v}(t, \overrightarrow{0})) \cdot \hat{\ell}]^{n}\right\rangle,
$$

where $\hat{\ell}=\vec{\ell} /|\vec{\ell}|$. Exploiting translation invariance, the second-order structure function hence reads

$$
S^{(2)}(\ell)=-2 \hat{\ell}_{i} \hat{\ell}_{j}\left\langle v_{j}(t, \vec{\ell}) v_{i}(t, \overrightarrow{0})-v_{j}(t, \overrightarrow{0}) v_{i}(t, \overrightarrow{0})\right\rangle
$$

and in terms of $G_{\alpha \beta}^{u u}$, one has

$$
\left\langle v_{\alpha}(t, \vec{\ell}) v_{\beta}(t, \overrightarrow{0})-v_{\alpha}(t, \overrightarrow{0}) v_{\beta}(t, \overrightarrow{0})\right\rangle=\int_{\mathbf{q}} G_{\alpha \beta}^{u u}(\omega, \vec{q})\left[e^{i \vec{q} \cdot \vec{\ell}}-1\right] .
$$

It then follows that

$$
\begin{aligned}
& S^{(2)}(\ell)=-2 \int_{\mathbf{q}} G_{\perp}^{u u}(\omega, \vec{q})\left[e^{i \vec{q} \cdot \vec{\ell}}-1\right]\left[1-\frac{(\hat{\ell} \cdot \vec{q})^{2}}{q^{2}}\right] \\
& =\gamma_{d} \int_{0}^{\infty} \frac{d \omega}{\pi} \int_{0}^{\infty} d q q^{d-1} \frac{\Gamma_{\perp}^{(0,2)}(\omega, \vec{q})-2 N_{\kappa}(\vec{q})}{\left|\Gamma_{\perp}^{(1,1)}(\omega, \vec{q})+R_{\kappa}(\vec{q})\right|^{2}} I_{d}(q \ell)
\end{aligned}
$$

with

$$
\begin{aligned}
\gamma_{d} & \equiv \frac{4 \pi^{(d-1) / 2}}{(2 \pi)^{d} \Gamma\left(\frac{d-1}{2}\right)} \\
I_{d}(v) & \equiv \int_{0}^{\pi} d \theta \sin ^{d} \theta\left[e^{i v \cos \theta}-1\right] \\
& =\int_{-1}^{1} d u\left(1-u^{2}\right)^{(d-1) / 2}\left[e^{i v u}-1\right] .
\end{aligned}
$$

In dimensions $d=2$ and $d=3$, the integrals $I_{d}(v)$ are given by

$$
\begin{aligned}
& I_{3}(v)=\frac{4}{v^{3}}\left[\sin v-v \cos v-\frac{v^{3}}{3}\right] \\
& I_{2}(v)=\pi\left[-\frac{1}{2}+\frac{1}{v} J_{1}(v)\right]
\end{aligned}
$$

where $J_{1}(v)$ denotes a type $J$ Bessel function of the first kind.

Again, let us focus on the inertial regime corresponding to $\ell \kappa \ll 1$. Since the integral in (54) is dominated by values of $q$ such that $q \ell$ is of order one, it is dominated by values of $q \gg \kappa$ in the inertial range. Accordingly, one can neglect in this regime the functions $N_{\kappa}(\vec{q})$ and $R_{\kappa}(\vec{q})$ that tend to zero rapidly and one obtains

$$
S^{(2)}(\ell) \stackrel{\ell \kappa \ll 1}{\simeq} \gamma_{d} \int_{0}^{\infty} \frac{d \omega}{\pi} \int_{0}^{\infty} d q q^{d-1} \frac{\Gamma_{\perp}^{(0,2)}(\omega, \vec{q})}{\left|\Gamma_{\perp}^{(1,1)}(\omega, \vec{q})\right|^{2}} I_{d}(q \ell) .
$$

\section{NPRG FLOW EQUATIONS AT THE LEADING ORDER APPROXIMATION}

In this section, we devise a simple approximation to study the stationary regime of the stirred NS equation, designed to provide a reliable description of the large distance properties of the system (that is, small wavenumber sector), and is therefore appropriate to investigate the existence of a fixed point of the NPRG flow. This approximation is similar to the approximation implemented in [84, 86], although presented differently, with emphasis on its condition of validity and limitations.

\section{A. Approximation scheme}

We here explain the principles and justifications of the approximation scheme. This technical subsection may be skipped in a first lecture. The NPRG flow of the effective action $\Gamma_{\kappa}$ is given by the exact equation (17). However, as this equation cannot be solved exactly, one has to devise some approximation. The standard approximation schemes within the NPRG framework are the derivative expansion 62] and the BMW approximation scheme [69].

The derivative expansion consists in an expansion of $\Gamma_{\kappa}$ in powers of gradients and time derivatives. It is tailored to provide an accurate description of the long time and large distance properties of the theory (zero external wave-vector and frequency sector), which encompass phase diagrams and critical exponents. However, if one needs the wave-vector and/or frequency dependencies of the vertex functions, one has to resort to a more sophisticated approximation scheme such as the BMW one. It consists in a closure at a given order $n$ of the hierarchy of flow equations for the $p$-point vertex functions $\Gamma_{\kappa}^{(p)}$ with $p \leq n$ by approximating the $\Gamma_{\kappa}^{(n+1)}$ and $\Gamma_{\kappa}^{(n+2)}$ vertices. For instance, for $n=2$, the set of flow equations (19) for the 2-point functions involves the vertices $\Gamma_{\kappa}^{(3)}$ and $\Gamma_{\kappa}^{(4)}$, that are approximated in the BMW scheme in such a way that they can be expressed in terms of derivatives of $\Gamma_{\kappa}^{(2)}$ thus yielding a closed set of equations. 
In this work, our aim is to compute the wave-vector dependent 2-point functions, in order to calculate the energy spectrum (50) and the second order structure function (58) of the stationary NS incompressible flow. Hence the derivative expansion is not appropriate and we resort to the BMW scheme. However, the standard implementation of this scheme, well-established for equilibrium problems [69], is hindered here by the symmetries. The reason is that, on the one hand, the BMW approximation requires an expansion of the vertex functions $\Gamma_{\kappa}^{(3)}$ and $\Gamma_{\kappa}^{(4)}$ in the internal wave-vector and frequency, but on the other hand, these dependencies are very much constrained through the Ward identities, ensuing in particular from the time-gauged Galilean symmetry, such that both are very difficult to conciliate a priori.

In fact, this obstacle has been successfully circumvented in another closely related nonequilibrium classical problem, the Kardar-Parisi-Zhang (KPZ) equation, which is a stochastic (Langevin) equation describing interface growth and roughening [77]. The KPZ equation turns out to share with the NS one a very similar invariance under time-gauged Galilean transformations, and also under time-gauged shifts (although of the field itself instead of the response field) [80]. This similarity is easily conceivable as the KPZ equation maps onto the Burgers equation. Thus the approximation scheme devised in the context of the KPZ equation [79 81] can be quite simply transposed to the NS equation.

For the KPZ equation, the solution consists in constructing an ansatz for $\Gamma_{\kappa}$ explicitly preserving the symmetries of the action, in particular the time-gauged Galilean symmetry. At second order (SO) of this scheme, the effective action $\Gamma_{\kappa}$ is truncated at quadratic order in the response field, while retaining for the 2-point functions an arbitrary dependence in wave-vectors and frequencies (and also in the field itself through arbitrary powers of the covariant time derivative). The 2-point correlation and response functions for the KPZ problem were calculated within the SO approximation in $d=1$ [80]. The existence of scaling forms for these functions could be proved analytically, and the related scaling functions in one dimension turned out to reproduce with an impressive agreement the exact results established in [110], including the finest detail of their tails. The SO approximation can therefore be roughly considered as a BMWlike scheme (for the field but not for the response field) rendered compatible with the KPZ symmetries.

From a numerical viewpoint, the SO approximation is demanding in dimensions larger than one. Two simpler approximations were thus proposed in 79 81]. They both consist in a simplification of the frequency sector, by either completely neglecting the frequency dependence of the 3-point vertices (leading order (LO) approximation) or approximating it (next-to-leading (NLO) approximation). The predictions obtained at NLO for some universal amplitude ratios of the KPZ problem in 81. were very accurately confirmed in $d=2$ in recent largescale simulations [11]. As for the LO approximation, which only retains the bare frequency dependence, but preserves the full wave-vector dependence of the 2-point functions, it clearly suffices in the KPZ problem to obtain the full phase diagram, including the strong-coupling rough phase. The related estimates for the critical exponents are in good agreement with the numerical ones in dimensions $d=2$ and $d=3[79,81]$. Let us underline that the values of the $\mathrm{KPZ}$ critical exponents are greatly improved at LO compared to those obtained within the Derivative Expansion [112], which is probably to be imputed to the derivative nature of the bare vertex.

As we merely consider, in the current work, the wavevector dependence of the 2-point functions of the NS problem, we choose to implement the LO approximation for the NS equation, which is achieved in the next section. For the NS effective action $\tilde{\Gamma}_{\kappa}$ in Eq. (27), it consists in

- (i) performing a field expansion in $\overline{\vec{u}}$ at order two

- (ii) keeping only the bare wave-vector and frequency dependencies of all $n$-point functions with $n \geq 3$

- (iii) preserving an arbitrary wave-vector dependence of the 2-point functions while restricting to their bare frequency dependence.

In this approach, all the symmetries of the theory are automatically encoded by writing the proposed ansatz for $\tilde{\Gamma}_{\kappa}$ in terms of Galilean scalars only. Notice that within the LO approximation, point (ii) implies that all $n$-point vertex functions with $n \geq 4$ vanish and point (iii) that $\tilde{\Gamma}_{\kappa}$ is also truncated at order two in $\vec{u}$ since the dependence in the $D_{t}$ covariant derivative is neglected. The LO ansatz is given by Eq. (59).

Let us comment on the validity/accuracy of the LO approximation. Point (ii) implies that this approximation is valid only for wave-vectors typically smaller than $\kappa$ and frequencies smaller than $\kappa^{1 / z}$ because keeping the bare wave-vector dependence of the $n$-point functions for $n \geq 3$ is equivalent to keeping their leading terms in a wave-vector expansion. Notice that once this expansion is performed, the frequency sector is entirely fixed by the symmetries gauged in time, see Sec. VII This expansion is certainly valid for the internal wave-vector $\vec{q}$ in Eq. (19) since it is suppressed for $|\vec{q}| \gg \kappa$ by the $\partial_{\kappa} \mathcal{R}_{\kappa}$ term but, a priori, is only justified for small external wave-vector, that is $|\vec{p}| \lesssim \kappa$ in Eq. (19). In fact, in most systems, including the KPZ growth, the flow of the 2-point functions actually stops when the external wave-vector becomes larger than the RG scale $\kappa$, a phenomenon called decoupling. Thus, the determination of the momentum and frequency dependencies remains accurate within the LO approximation because (a) when $\kappa \gtrsim|\vec{p}|$ (and $\kappa \gtrsim \nu^{1 / z}$ ) the LO approximation is controlled and (b) when $\kappa$ becomes smaller than $|\vec{p}|$, the flow almost stops and although the LO approximation is not valid in this region of wave-numbers, this has negligible impact on the two-point functions. 
However, as stressed in the following, the NS problem is very peculiar because the RG flow of the 2-point functions does not satisfy the decoupling property of the large wave-vector sector. Thus a complementary scheme is necessary to determine the wave-vector dependence of $\Gamma_{\kappa}^{(2)}(\vec{p})$ at finite $\vec{p}$ when $\kappa \rightarrow 0$. The LO approximation analyzed below is therefore only valid in the limit $|\vec{p}| \rightarrow 0$ when $\kappa \rightarrow 0$, which is sufficient to determine the fixed point structure and critical exponents, but not to investigate multiscaling. On the other hand, as shown in Sec. VII exact RG flow equations can be derived in the large $\vec{p}$ sector, relying on the very constraining gauge symmetries of the NS field theory, which can take over from the LO equations when $\kappa$ becomes smaller than $|\vec{p}|$. In fact, a source for the multiscaling behavior of the $n$ point functions precisely emerges in the NPRG framework from the non-decoupling of the large $\vec{p}$ sector and the associated nontrivial behavior in both $|\vec{p}|$ and $\nu$ (see Sec. VII).

\section{B. LO ansatz}

As explained in the previous section, one can construct an ansatz for $\tilde{\Gamma}_{\kappa}$ in (27) which explicitly preserves the time-gauged Galilean symmetry by using as building blocks Galilean scalars (see Ref. [80] for detail). The shift gauged symmetry can then also be simply enforced. At LO, the functional $\tilde{\Gamma}_{\kappa}$ is truncated at quadratic order in the response field $\overline{\vec{u}}$ and moreover, the (non-bare) frequency dependence (i.e. a dependence in the covariant time derivative $D_{t}$ of the running functions $f_{\kappa, \alpha \beta}^{\nu, D}$ below) is neglected. Hence the LO ansatz simply reads

$$
\begin{aligned}
\tilde{\Gamma}_{\kappa}[\vec{u}, \overrightarrow{\vec{u}}] & =\int_{t, \vec{x}, \vec{x}^{\prime}}\left\{\bar{u}_{\alpha}(t, \vec{x}) f_{\kappa, \alpha \beta}^{\nu}\left(\vec{x}-\vec{x}^{\prime}\right) u_{\beta}\left(t, \vec{x}^{\prime}\right)\right. \\
& \left.-\bar{u}_{\alpha}(t, \vec{x}) f_{\kappa, \alpha \beta}^{D}\left(\vec{x}-\vec{x}^{\prime}\right) \bar{u}_{\beta}\left(t, \vec{x}^{\prime}\right)\right\} .
\end{aligned}
$$

To further specify the running functions $f_{\kappa, \alpha \beta}^{\nu, D}$, let us introduce the notation for the vertex functions

$$
\begin{aligned}
& \Gamma_{\alpha_{1}, \ldots, \alpha_{m}, \beta_{1}, \ldots, \beta_{n}}^{(m, n)}\left(\mathbf{x}_{1}, \ldots, \mathbf{x}_{m}, \overline{\mathbf{x}}_{1}, \ldots, \overline{\mathbf{x}}_{n}\right) \\
& =\frac{\delta^{n+m} \Gamma_{\kappa}}{\delta u_{\alpha_{1}}\left(\mathbf{x}_{1}\right) \ldots \delta u_{\alpha_{m}}\left(\mathbf{x}_{m}\right) \delta \bar{u}_{\beta_{1}}\left(\overline{\mathbf{x}}_{1}\right) \ldots \delta \bar{u}_{\beta_{n}}\left(\overline{\mathbf{x}}_{n}\right)}
\end{aligned}
$$

where from now on, the explicit index $\kappa$ is dropped for the vertex functions $\Gamma^{(m, n)} \equiv \Gamma_{\kappa}^{(m, n)}$ and for the running functions $f_{\alpha \beta}^{\nu, D} \equiv f_{\kappa, \alpha \beta}^{\nu, D}$. The Fourier transforms of the $\Gamma^{(m, n)}$ only depend on $n+m-1$ wave-vectors and frequencies because of translation invariance.

The dependence of the running functions $f_{\alpha \beta}^{\nu}$ and $f_{\alpha \beta}^{D}$ in $\vec{x}-\vec{x}^{\prime}$ is in fact through gradients as can be inferred from the Ward identities (E4) and (E12). The latters imply that these two functions vanish at zero wave-vector

$$
f_{\alpha \beta}^{\nu}(\vec{p}=\overrightarrow{0})=f_{\alpha \beta}^{D}(\vec{p}=\overrightarrow{0})=0 .
$$

The initial conditions of the flow at scale $\kappa=\eta^{-1}$ for the two running functions are

$$
\begin{aligned}
& \left.f_{\alpha \beta}^{D}\left(\vec{x}-\vec{x}^{\prime}\right)\right|_{\kappa=\eta^{-1}}=0 \\
& \left.f_{\alpha \beta}^{\nu}\left(\vec{x}-\vec{x}^{\prime}\right)\right|_{\kappa=\eta^{-1}}=-\nu \delta_{\alpha \beta} \nabla_{x}^{2}\left(\delta^{(d)}\left(\vec{x}-\vec{x}^{\prime}\right)\right)
\end{aligned}
$$

to recover the original NS action (5) at the microscopic scale.

The calculation of the 2-point functions from the LO ansatz is straightforward. At vanishing fields and in Fourier space, one obtains

$$
\begin{aligned}
& \tilde{\Gamma}_{\alpha \beta}^{(2,0)}(\omega, \vec{p})=0 \\
& \tilde{\Gamma}_{\alpha \beta}^{(1,1)}(\omega, \vec{p})=f_{\alpha \beta}^{\nu}(\vec{p}) \\
& \tilde{\Gamma}_{\alpha \beta}^{(0,2)}(\omega, \vec{p})=-2 f_{\alpha \beta}^{D}(\vec{p}) .
\end{aligned}
$$

Within the LO approximation, all vertex functions of order $m+n \geq 4$ vanish, and the only non-zero 3-point vertex function is the bare one, which reads in Fourier space

$$
\Gamma_{\alpha \beta \gamma}^{(2,1)}\left(\omega_{1}, \vec{p}_{1}, \omega_{2}, \vec{p}_{2}\right)=-i \lambda\left(p_{2}^{\alpha} \delta_{\beta \gamma}+p_{1}^{\beta} \delta_{\alpha \gamma}\right) .
$$

\section{Derivation of the LO flow equations}

In this section, we derive the $\mathrm{LO}$ flow equations of the two running functions $f_{\alpha \beta}^{\nu}$ and $f_{\alpha \beta}^{D}$. In fact, for incompressible flows, only the transverse sector plays a role, which means that only their transverse components, denoted $f_{\perp}^{\nu}$ and $f_{\perp}^{D}$, are eventually needed. The flow equations of $f_{\perp}^{\nu}$ and $f_{\perp}^{D}$ are proportional to the projection in the transverse sector of the flow equations of the 2-point functions $\Gamma_{\alpha \beta}^{(1,1)}(0, \vec{p})$ and $\Gamma_{\alpha \beta}^{(0,2)}(0, \vec{p})$, which are given in matrix form by Eq. (19) evaluated at zero external frequency. At $\mathrm{LO}$, the matrices $\Gamma_{\kappa, i j}^{(4)}$ entering these equations are zero and only one 3 -point vertex, the bare one $\Gamma^{(2,1)}$, contributes in the matrices $\Gamma_{\kappa, i}^{(3)}$. The transverse components of the propagator in the $\vec{u}, \overline{\vec{u}}$ sector (30) are given within the $\mathrm{LO}$ approximation, i.e. with the ansatz (63), by

$$
\begin{aligned}
& G_{\perp}^{\bar{u} \bar{u}}(\omega, \vec{p})=0 \\
& G_{\perp}^{u \bar{u}}(\omega, \vec{p})=\frac{1}{-i \omega+\tilde{f}_{\perp}^{\nu}(\vec{p})} \\
& G_{\perp}^{u u}(\omega, \vec{p})=\frac{2 \tilde{f}_{\perp}^{D}(\vec{p})}{\omega^{2}+\left(\tilde{f}_{\perp}^{\nu}(\vec{p})\right)^{2}}
\end{aligned}
$$

where

$$
\begin{aligned}
& \tilde{f}_{\perp}^{\nu}(\vec{p}) \equiv f_{\perp}^{\nu}(\vec{p})+R_{\kappa}(\vec{q}) \\
& \tilde{f}_{\perp}^{D}(\vec{p}) \equiv f_{\perp}^{D}(\vec{p})+N_{\kappa}(\vec{q}) .
\end{aligned}
$$

One has to compute the trace of the matrix product (19), and project the result onto the transverse sector. In the 
obtained expression, since the frequency dependence remains the bare one at LO, the integral over the internal frequency $\omega$ can be analytically carried out. These cal- culations are detailed in Appendix B. The resulting flow equations for the two running functions $f_{\perp}^{\nu}$ and $f_{\perp}^{D}$ are given by

$$
\begin{aligned}
\partial_{s} f_{\perp}^{\nu}(\vec{p}) & =\frac{\lambda^{2}}{(d-1)} \int_{\vec{q}}\left\{\frac{\partial_{s} R_{\kappa}(\vec{q}) \tilde{f}_{\perp}^{D}(\vec{p}+\vec{q})}{\tilde{f}_{\perp}^{\nu}(\vec{p}+\vec{q})\left(\tilde{f}_{\perp}^{\nu}(\vec{q})+\tilde{f}_{\perp}^{\nu}(\vec{p}+\vec{q})\right)^{2}}\left[\left(-\vec{p}^{2}+\frac{(\vec{p} \cdot(\vec{p}+\vec{q}))^{2}}{(\vec{p}+\vec{q})^{2}}\right)(d-1)-2 \vec{p} \cdot \vec{q}\left(1-\frac{(\vec{p} \cdot \vec{q})^{2}}{\vec{q}^{2} \vec{p}^{2}}\right)\right]\right. \\
& +\frac{1}{\tilde{f}_{\perp}^{\nu}(\vec{q})\left(\tilde{f}_{\perp}^{\nu}(\vec{q})+\tilde{f}_{\perp}^{\nu}(\vec{p}+\vec{q})\right)}\left[\partial_{s} R_{\kappa}(\vec{q}) \frac{\tilde{f}_{\perp}^{D}(\vec{q})\left(2 \tilde{f}_{\perp}^{\nu}(\vec{q})+\tilde{f}_{\perp}^{\nu}(\vec{p}+\vec{q})\right)}{\tilde{f}_{\perp}^{\nu}(\vec{q})\left(\tilde{f}_{\perp}^{\nu}(\vec{q})+\tilde{f}_{\perp}^{\nu}(\vec{p}+\vec{q})\right)}-\partial_{s} N_{\kappa}(\vec{q})\right] \\
& \left.\times\left[\left(-\vec{p}^{2}+\frac{(\vec{p} \cdot \vec{q})^{2}}{\vec{q}^{2}}\right)(d-1)+2 \frac{\vec{p} \cdot(\vec{p}+\vec{q})}{(\vec{q}+\vec{p})^{2}}\left(\vec{q}^{2}-\frac{(\vec{p} \cdot \vec{q})^{2}}{\vec{p}^{2}}\right)\right]\right\} \\
\partial_{s} f_{\perp}^{D}(\vec{p}) & =-\frac{\lambda^{2}}{2(d-1)} \int_{\vec{q}}\left\{\frac { \tilde { f } _ { \perp } ^ { D } ( \vec { q } + \vec { p } ) } { \tilde { f } _ { \perp } ^ { \nu } ( \vec { p } + \vec { q } ) \tilde { f } _ { \perp } ^ { \nu } ( \vec { q } ) ( \tilde { f } _ { \perp } ^ { \nu } ( \vec { q } ) + \tilde { f } _ { \perp } ^ { \nu } ( \vec { p } + \vec { q } ) ) } \left[\partial_{s} R_{\kappa}(\vec{q}) \frac{\tilde{f}_{\perp}^{D}(\vec{q})\left(2 \tilde{f}_{\perp}^{\nu}(\vec{q})+\tilde{f}_{\perp}^{\nu}(\vec{p}+\vec{q})\right)}{\tilde{f}_{\perp}^{\nu}(\vec{q})\left(\tilde{f}_{\perp}^{\nu}(\vec{q})+\tilde{f}_{\perp}^{\nu}(\vec{p}+\vec{q})\right)}\right.\right. \\
& \left.\left.\times\left[\left(2 \vec{p}^{2}+\frac{(\vec{p} \cdot(\vec{p}+\vec{q}))^{2}}{(\vec{p}+\vec{q})^{2}}-\frac{(\vec{p} \cdot \vec{q})^{2}}{\vec{q}^{2}}\right)(d-1)+2 \frac{1}{\vec{q}^{2}(\vec{p}+\vec{q})^{2}}(\vec{q})^{2}-\frac{(\vec{p} \cdot \vec{q})^{2}}{\vec{p}^{2}}\right)\left(\vec{p}^{2} \vec{p} \cdot \vec{q}+2(\vec{p} \cdot \vec{q})^{2}-\vec{p}^{2} \vec{q}^{2}\right)\right]\right\}
\end{aligned}
$$

where $\partial_{s} \equiv \kappa \partial_{\kappa}$.

\section{Dimensionless LO flow equations}

As we seek the fixed point solution of the flow equations, we work with dimensionless and renormalized quantities, as defined in Sec. IV] Let us determine the dimensions of the terms entering the LO ansatz (27/59). According to the definitions (31) and (32), the two functions $\hat{r}\left(q^{2} / \kappa^{2}\right)$ and $\hat{n}\left(q^{2} / \kappa^{2}\right)$ introduced in Eqs. (8) and (11) are dimensionless, and the flow of the regulator terms in Eqs. (67) and (68) are given by

$$
\begin{aligned}
& \partial_{s} R_{\kappa}(\vec{q})=\nu_{\kappa} q^{2}\left(-\eta_{\kappa}^{\nu} \hat{r}\left(\hat{q}^{2}\right)-2 \hat{q}^{2} \partial_{\hat{q}^{2}} \hat{r}\left(\hat{q}^{2}\right)\right) \\
& \partial_{s} N_{\kappa}(\vec{q})=D_{\kappa} \hat{q}^{2}\left(-\left(\eta_{\kappa}^{D}+2\right) \hat{n}\left(\hat{q}^{2}\right)-2 \hat{q}^{2} \partial_{\hat{q}^{2}} \hat{n}\left(\hat{q}^{2}\right)\right) .
\end{aligned}
$$

Then, according to Eq. (59), the dimensions of the running functions $f_{\alpha \beta}^{\nu}$ and $f_{\alpha \beta}^{D}$ are the same as the ones of $R_{\kappa, \alpha \beta}$ and $N_{\kappa, \alpha \beta}$ in Eqs. (31) and (32) and we thus define the dimensionless functions $\hat{h}^{\nu}$ and $\hat{h}^{D}$ as

$$
f_{\perp}^{\nu}(\vec{p})=\nu_{\kappa} \kappa^{2} \hat{p}^{2} \hat{h}^{\nu}(\hat{\vec{p}}) \quad \text { and } \quad f_{\perp}^{D}(\vec{p})=D_{\kappa} \hat{p}^{2} \hat{h}^{D}(\hat{\vec{p}}) .
$$

Their flow equations are given by

$$
\begin{aligned}
& \partial_{s} \hat{h}^{\nu}(\hat{\vec{p}})=\eta_{\kappa}^{\nu} \hat{h}^{\nu}(\hat{\vec{p}})+\hat{\vec{p}} \partial_{\hat{\vec{p}}} \hat{h}^{\nu}(\hat{\vec{p}})+\nu_{\kappa}^{-1} \frac{\partial_{s} f_{\perp}^{\nu}(\vec{p})}{p^{2}} \\
& \partial_{s} \hat{h}^{D}(\hat{\vec{p}})=\left(\eta_{\kappa}^{D}+2\right) \hat{h}^{D}(\hat{\vec{p}})+\hat{\vec{p}} \partial_{\hat{\vec{p}}} \hat{h}^{D}(\hat{\vec{p}})+D_{\kappa}^{-1} \frac{\partial_{s} f_{\perp}^{D}(\vec{p})}{\hat{p}^{2}}
\end{aligned}
$$

with the substitutions for dimensionless quantities in the flow equations (67) and (68) for $\partial_{s} f_{\perp}^{\nu}(\vec{p})$ and $\partial_{s} f_{\perp}^{D}(\vec{p})$.

\section{FIXED POINT SOLUTIONS}

One has to integrate the two flow equations (71) to determine the scale evolution of the two running functions $h^{\nu}(\hat{\vec{p}})$ and $h^{D}(\hat{\vec{p}})$. For simplicity, the two running exponents $\eta_{\kappa}^{\nu}$ and $\eta_{\kappa}^{D}$ are fixed to the values (43) in $d=3$ and (46) in $d=2$ at any $\kappa$, that is all along the flow (note that $\partial_{s} \hat{\lambda}_{\kappa}=0$ for all $\kappa$ once the exponents are fixed). We checked that it does not affect the critical properties at the fixed point. These flow equations are integrated numerically from the initial conditions $\hat{h}^{D}(\vec{p})=0$ and $\hat{h}^{\nu}(\vec{p})=1$, with different values of $\hat{\lambda}$ and different values of the $a$ parameter in Eq. (12). The detail of the numerical procedure is summarized in Appendix D. We observe that the flow always reaches a (fully attractive) fixed point without fine-tuning any parameter, and independently of the initial conditions, both in $d=3$ and in $d=2$. Hence the corresponding stationary regime is universal.

\section{A. Fixed point functions $\hat{h}^{\nu}$ and $\hat{h}^{D}$}

Along the flow, the two functions $\hat{h}^{\nu, D}$ are smoothly deformed to acquire a fixed form, which is illustrated on the example of the function $\hat{h}^{\nu}$ in $d=2$ and $d=3$ in Fig.1. The fixed point profile of the two functions in both dimensions is displayed in logarithmic scales in Fig. 2. This figure shows that both functions decay algebraically at large wave-number. We determined the corresponding decay exponents and observed that they deviate from the 

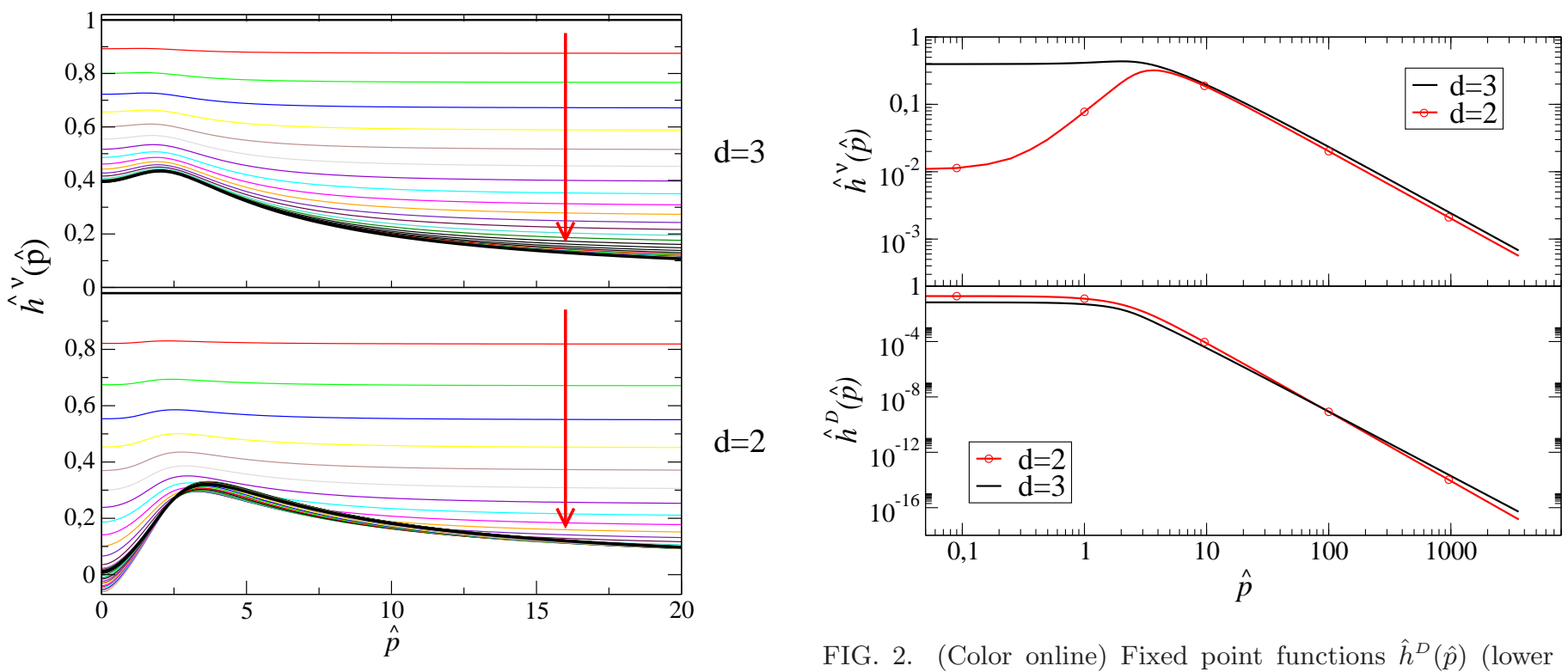

FIG. 1. (Color online) RG evolution of the dimensionless running function $\hat{h}^{\nu}(\hat{p})$ in dimensions $d=3$ (upper panel) and $d=2$ (lower panel) starting from the initial condition $\hat{h}^{\nu}(\hat{p})=1$ at the microscopic scale $\kappa=\eta^{-1}$. The red arrow corresponds to decreasing RG scales $\kappa$ and the black thick line to the fixed point function.

expected (dimensional) scalings. We indeed found

$$
\hat{h}^{\nu}(\hat{p}) \sim \hat{p}^{-\eta^{\nu}+\alpha} \quad \text { and } \quad \hat{h}^{D}(\hat{p}) \sim \hat{p}^{-\left(\eta^{D}+2\right)+\beta}
$$

where $\alpha$ and $\beta$ are the deviations from the dimensional (K41 or $\mathrm{KB}$ ) scalings, with the estimated values $\alpha \simeq$ $\beta \simeq 0.33$ in $d=3$ and $\alpha \simeq \beta \simeq 1.00$ in $d=2$. The values found are in agreement with the values reported in $d=3$ in Ref. [84]. They are also very close to the values that can be estimated from the Figures 3 and 7 of Ref. [86], which confirms that the large $\epsilon$ regime in this work corresponds in fact to the fixed-point with localized forcing, although this was not noticed by the authors. It was shown in particular in [84] that these exponents are independent of the choice of the stirring profile. In fact, one can prove by inspection of the regime $p \gg \kappa$ of the LO flow equations (67) and (68) that the exponent $\alpha$ is exactly $1 / 3$ in $d=3$ and 1 in $d=2$. Moreover, we found numerically that $\beta \simeq 1 / 3$ in $d=3$ and $\beta \simeq 1$ in $d=2$, if not exactly at least very precisely [113]. Let us now probe the effect of these deviations on physical observables.
FIG. 2. (Color online) Fixed point functions $\hat{h}^{D}(\hat{p})$ (lower panel) and $\hat{h}^{\nu}(\hat{p})$ (upper panel) in dimensions $d=3$ (black curves) and $d=2$ (red curves). Both horizontal and vertical axes are in logarithmic scales.

\section{B. Energy spectrum}

Within the LO approximation, the 2-point functions are given by

$$
\begin{aligned}
& \Gamma_{\perp}^{(0,2)}(\omega, \vec{q})=-2 D_{\kappa} \hat{q}^{2} \hat{h}^{D}(\hat{\vec{q}}) \\
& \Gamma_{\perp}^{(1,1)}(\omega, \vec{q})=\nu_{\kappa} \kappa^{2}\left(i \hat{\omega}+\hat{q}^{2} \hat{h}^{\nu}(\hat{q})\right) .
\end{aligned}
$$

Inserting these expressions into Eq. (50) and performing the integral over the frequency, one obtains for the energy spectrum

$$
E^{(d)}(\vec{p}) \simeq \frac{2 \pi^{d / 2}}{\Gamma(d / 2)}(d-1) p^{d-1} \frac{D_{\kappa}}{\nu_{\kappa} \kappa^{2}} \frac{\hat{h}^{D}(\hat{p})}{\hat{h}^{\nu}(\hat{p})} .
$$

The corresponding energy spectra in $d=3$ and $d=2$ are displayed on Fig. 3. At small wave-vector, the two functions $\hat{h}^{\nu, D}(\hat{p})$ tend to a finite constant. It follows that the energy spectra grow as

$$
E^{(d)}(\vec{p}) \sim p^{d-1} \kappa^{-\eta^{D}+\eta^{\nu}-2}
$$

with the power law $p^{d-1}$ reflecting equipartition of energy. At large wave-vector, the two functions $\hat{h}^{\nu, D}(\hat{p})$ follow the asymptotics (72) and the energy spectra in both dimensions thus decay algebraically as

$$
\begin{aligned}
E^{(d)}(\vec{p}) & \sim p^{d-3-\eta^{D}+\eta^{\nu}}\left(\frac{p}{\kappa}\right)^{\beta-\alpha} \\
& = \begin{cases}p^{-5 / 3}\left(\frac{p}{\kappa}\right)^{\beta-\alpha} & \text { in } d=3 \\
p^{-3}\left(\frac{p}{\kappa}\right)^{\beta-\alpha} & \text { in } d=2\end{cases}
\end{aligned}
$$

using Eqs. (43) and (46). 
In $d=3$, we hence recover the Kolmogorov scaling when $\alpha=\beta$ which describes the decay of the energy spectrum when all the energy is dominantly transferred towards the small scale in the direct cascade.

Let us compute at LO approximation the rate of dissipated power at the microscopic scale in $d=3$ and check that the presence of deviations from dimensional scalings does not alter the analysis of Sec. IVA The average dissipated power per unit mass (40) is expressed as

$$
\left\langle\epsilon_{\text {dis }}^{1 / \eta}\right\rangle=-\nu(d-1) \int_{\omega, \vec{q}} q^{2} \frac{\Gamma_{\kappa}^{(0,2)}(\omega, \vec{q})-2 N_{\kappa}(\vec{q})}{\left|\Gamma_{\kappa}^{(1,1)}(\omega, \vec{q})+R_{\kappa}(\vec{q})\right|^{2}} .
$$

For $\vec{q}$ in the inertial range, $N_{\kappa}$ and $R_{\kappa}$ are negligible, and within the LO approximation one obtains

$$
\left\langle\epsilon_{\mathrm{dis}}^{1 / \eta}\right\rangle=\nu \frac{\kappa^{d} D_{\kappa}}{\nu_{\kappa}} \frac{d-1}{2^{d-1} \pi^{d / 2} \Gamma(d / 2)} \int_{0}^{1 /(\kappa \eta)} d \hat{q} \hat{q}^{d+1} \frac{\hat{h}^{D}(\hat{q})}{\hat{h}^{\nu}(\hat{q})} .
$$

This integral is UV divergent in $d>2$ without an UV cutoff, and hence dominated by the UV scale $\eta^{-1}$. Indeed, for $\kappa \ll \eta^{-1}$, the two functions $\hat{h}^{D}$ and $\hat{h}^{\nu}$ follow the asymptotics (72) and thus in $d=3$

$$
\begin{aligned}
\left\langle\epsilon_{\mathrm{dis}}^{1 / \eta}\right\rangle & \propto \kappa^{d-\eta^{D}+\eta^{\nu}} \int_{0}^{1 /(\kappa \eta)} d \hat{q} \hat{q}^{1 / 3+\beta-\alpha} \\
& \propto \eta^{-4 / 3-\beta+\alpha} \kappa^{\alpha-\beta},
\end{aligned}
$$

and is independent of $\kappa$ for $\alpha \simeq \beta$. This corroborates the analysis of Sec. IVA Thus, the flux of energy is constant in the inertial range between the injection scale $\kappa^{-1}$ and the dissipation scale $\eta$, as expected in three-dimensional turbulence.

In $d=2$, we find according to Eq. (76) that the decay of the energy spectrum in the direct cascade is steeper (slope -3 ) in $d=2$ than in $d=3$. This is expected since part of the energy is transferred in the inverse cascade in $d=2$. We recover the $p^{-3}$ decay in the direct cascade predicted by KB theory with no (or very small) corrections when $\alpha \simeq \beta$. One can check explicitly that the mean rate of dissipated vorticity (44) is indeed dominated by the UV scale $\eta^{-1}$ (behaving as $\eta^{-2}$ ) and thus independent of $\kappa$, confirming the analysis of Sec. IVA, We remind that we do not have access here to the inverse cascade since the integral scale is merged with the (inverse) RG scale and sent to infinity with it, that is the energy is effectively injected at the boundaries of the system. To investigate the inverse cascade, the integral scale (energy injection scale) should be kept fixed while the volume scale, identified in that case with the RG scale, should diverge in the limit $\kappa \rightarrow 0$. This important study is left for future work.

Both these results seem to indicate that, although the 2-point functions do display substancial deviations to their dimensional scalings, these deviations cancel out (or almost) for the energy spectrum, which is in agreement with experimental and numerical results which find very small (if any) corrections [4]. However, as explained previously, the LO approximation is not appropriate to study this regime, since it is based on an expansion of all wave-vectors, including the external ones, in powers of wave-vectors over $\kappa$. Definite conclusions are postponed to Sec. VII.

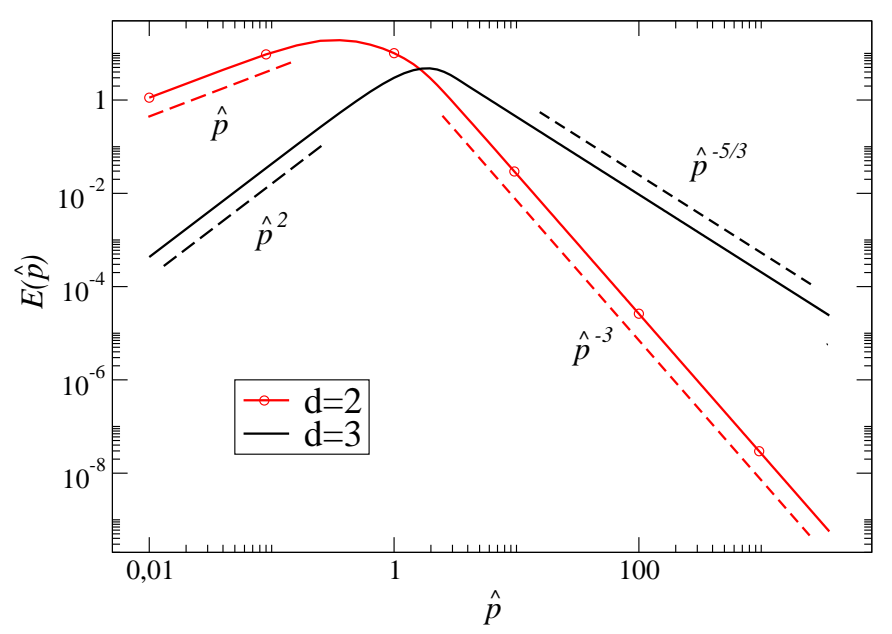

FIG. 3. (Color online) Energy spectrum (multiplied by the appropriate power $\kappa^{-5 / 3}$ in $d=3$ and $\kappa^{-3}$ in $d=2$ ) of the turbulent flow in dimensions $d=3$ and $d=2$ as a function of the dimensionless wave-number $\hat{p}=|\vec{p}| / \kappa$. The dashed lines are guidelines for the eyes.

\section{Second-order structure function}

Within the LO approximation, one obtains for the second order structure function (58) inserting the expressions (73)

$$
S^{(2)}(\ell) \simeq-\gamma_{d} \frac{D_{\kappa}}{\nu_{\kappa}} \kappa^{d-2} \int_{0}^{\infty} d \hat{q} \hat{q}^{d-1} \frac{\hat{h}^{D}(\hat{q})}{\hat{h}^{\nu}(\hat{q})} I_{d}(\kappa \hat{q} \ell) .
$$

Let us determine the behavior of this quantity within the inertial regime, which corresponds to the limit $\kappa \ell \ll 1$. Performing the change of variables $x=\kappa \hat{q} \ell$, one obtains

$$
\begin{aligned}
S^{(2)}(\ell) & \simeq-\gamma_{d} \frac{D_{\kappa}}{\nu_{\kappa}} \kappa^{d-2}(\kappa \ell)^{-d} \int_{0}^{\infty} d x x^{d-1} \frac{\hat{h}^{D}\left(\frac{x}{\kappa \ell}\right)}{\hat{h}^{\nu}\left(\frac{x}{\kappa \ell}\right)} I_{d}(x) \\
& \sim-\gamma_{d} \kappa^{\alpha-\beta} \ell^{2-d+\eta^{D}-\eta^{\nu}+\alpha-\beta} \\
& \times \int_{0}^{\infty} d x x^{d-3-\eta^{D}+\eta^{\nu}+\beta-\alpha} I_{d}(x) .
\end{aligned}
$$

where in the second equality the asymptotics (72) are used. The integral over $x$ is both IR and UV finite. 
Hence, in $d=3$,

$$
S^{(2)}(\ell) \sim-\gamma_{d} \kappa^{\alpha-\beta} \ell^{2 / 3+\alpha-\beta} \int_{0}^{\infty} d x x^{-5 / 3+\beta-\alpha} I_{d}(x),
$$

the Kolmogorov scaling is again recovered (or receives a very small correction) for $\alpha \simeq \beta$. In $d=2$,

$$
S^{(2)}(\ell) \sim-\gamma_{d} \kappa^{\alpha-\beta} \ell^{2+\alpha-\beta} \int_{0}^{\infty} d x x^{-3+\beta-\alpha} I_{d}(x),
$$

and no deviation (or very small effect) from the KB scaling is found when $\alpha \simeq \beta$. Both these results are again not in contradiction with experimental and numerical results [4].

As previously, one should be cautious with these results because the regime of wave-numbers much larger than $\kappa$ is not controlled in the $\mathrm{LO}$ approximation. Let us also point out that finding standard scaling for $S^{(2)}$ does not entail that the higher-order structure functions $S^{(n)}, n>$ 3 do not exhibit intermittency either, because this result for $S^{(2)}$ relies on compensations which are not likely to be generic. This is further discussed in Sec. VII

\section{Large wave-number sector and limit of the LO approximation}

In this section, we analyze the large wave-number limit of the flow equations (71). This analysis unveils that the nonlinear parts of these equations given by Eqs. (67) and (68) do not become negligible at the fixed point compared to the linear (dimensional) parts for large external wavevectors $\vec{p}$. This means that the large wave-number sector does not decouple from the flow when $\kappa \ll|\vec{p}|$, which is a very unusual property. As a consequence, the existence of the fixed point does not lead to the usual scale invariance, as encountered in ordinary critical phenomena. Indeed, the non-decoupling entails that the large wavevector sector is not determined by the small wave-vector one, that is the large $|\vec{p}|$ behavior - the exponents of the algebraic tails of the correlation functions - is not fixed in terms of the anomalous dimensions $\left(\eta^{\nu}\right.$ and $\left.\eta^{D}\right)$ and can develop deviations from them.

These deviations cannot be reliably computed at LO because this approximation is fully justified only when all the wave-numbers are small. As already explained, whereas this expansion is always valid for the internal wave-vector because of the presence of the regulator term $\partial_{\kappa} \mathcal{R}_{\kappa}$ which effectively cuts off the contributions $|\vec{q}| \gg \kappa$, it is justified only for small external wavevectors $\vec{p}$. Hence, one has to devise an alternative approximation to properly describe the large wave-number sector. This is achieved in Sec. VIII In fact, we prove in this section that the non-decoupling is a real feature of the exact NPRG flow of the NS problem and not an artifact of the LO approximation. Therefore, it is instructive to first understand how this non-decoupling works on the example of the LO flow equations.
To this aim, we now study the large $\vec{p}$ sector of the flow equations (71). We observed that the dimensionless running functions $\hat{h}^{\nu, D}$ reach a fixed point for $\kappa \ll \eta^{-1}$, which means by definition $\partial_{s} \hat{h}^{\nu}(\hat{p})=\partial_{s} \hat{h}^{D}(\hat{p})=0$. We concentrate in the following on this regime. Let us assume for a moment that, as it generally occurs, the nonlinear terms of the flow equations (71), denoted $\mathcal{L}^{\nu} \equiv \partial_{s} f_{\perp}^{\nu}(\vec{p}) /\left(\nu_{\kappa} p^{2}\right)$ and $\mathcal{L}^{D} \equiv \partial_{s} f_{\perp}^{D}(\vec{p}) /\left(D_{\kappa} \hat{p}^{2}\right)$ and which explicit expressions are given by (67) and (68), become negligible in the large wave-number limit $|\vec{p}| \gg \kappa$ compared to the linear terms, that is, they decouple. One then deduces that the general solutions at the fixed point of the remaining homogeneous (linear) parts of the flow equations are the scaling forms

$$
\hat{h}_{*}^{\nu}(\hat{p})=\hat{p}^{-\eta^{\nu}} \zeta^{\nu} \quad \text { and } \quad \hat{h}_{*}^{D}(\hat{p})=\hat{p}^{-\left(\eta^{D}+2\right)} \zeta^{D}
$$

where $\zeta^{\nu, D}$ are constants. Had we considered wave-vector and frequency dependent running functions, we would have obtained, e.g. $\hat{h}^{\nu}(\hat{\omega}, \hat{p})=\hat{p}^{-\eta^{\nu}} \zeta\left(\hat{\omega} / \hat{p}^{2-\eta^{\nu}}\right)$. We now show that this leads to a contradiction, that is, these scaling solutions are incompatible with the assumed decoupling of the large wave-number sector. For this, the leading contribution in $\hat{p}$ of each nonlinear term $\mathcal{L}^{\nu}$ and $\mathcal{L}^{D}$ can be analytically determined substituting the two functions $\hat{h}^{\nu, D}$ with the scaling solutions (84). One obtains that the dominant contributions of $\mathcal{L}^{\nu}$ and $\mathcal{L}^{D}$ are respectively $\hat{p}^{-\eta^{\nu}}$ and $\hat{p}^{-\left(\eta^{D}+2\right)}$. Hence, they are not negligible (sub-dominant) compared to the linear terms, but of the same order. One concludes that in LO approximation the nonlinear parts of the flow equations do not decouple at the fixed point, which invalidates the general scaling solutions (84).

This non-decoupling property entails that the existence of the fixed point does not generate scale invariance as usual (in critical phenomena). As a matter of fact, we found in the previous section that the two functions $\hat{h}^{\nu, D}$ do decay algebraically, but not with the dimensional exponents (84). Instead, they exhibit the deviations $\alpha$ and $\beta$ to these scalings following the asymptotics Eqs. (72). Of course, the realm of the non-decoupling property for the exact NS flow equations cannot be asserted at the LO level and the obtained values of these deviations are not to be trusted. However, we now specifically address the large wave-number regime, and prove that non-decoupling indeed occurs in the exact NPRG flow for NS.

\section{EXACT NPRG FLOW EQUATIONS IN THE LARGE WAVE-NUMBER REGIME}

The LO approximation is justified and expected to be accurate in the small wave-number regime $|\vec{p}| \lesssim \kappa$. In this section, we devise an alternative and complementary approximation for the large wave-number regime, $|\vec{p}| \gtrsim \kappa$ which becomes exact in the limit $|\vec{p}| \gg \kappa$, or equivalently in the limit $\kappa \rightarrow 0$ for any fixed external wave-vector and 
(a)

(c)

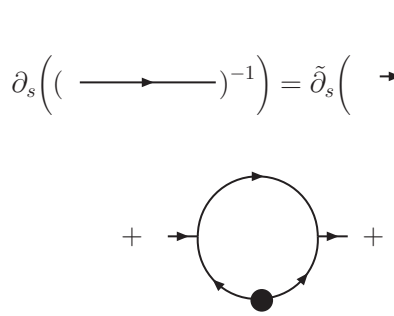

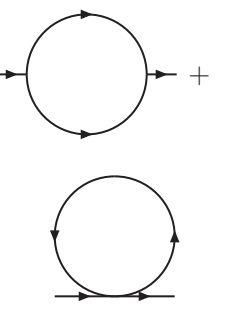

(d) (b)

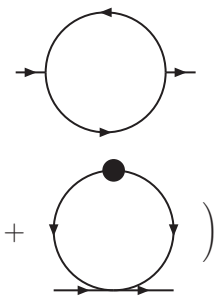

(e)
FIG. 4. Diagrammatic representation of the exact flow equation of $\Gamma_{\perp}^{(1,1)}(\nu, \vec{p})$, with $\tilde{\partial}_{s} \equiv \partial_{s} R_{\kappa} \frac{\partial}{\partial R_{\kappa}}+\partial_{s} N_{\kappa} \frac{\partial}{\partial N_{\kappa}}$. The combinatorial factors are not explicitly written, and diagrams involving $\Gamma_{\kappa}^{(n, 0)}$ vertices are omitted since they are vanishing. (a)

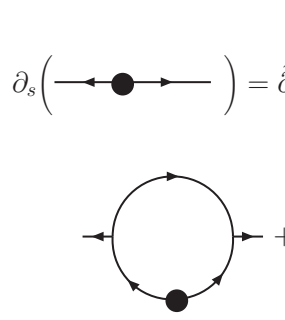

(c)

$(d)$ (b)
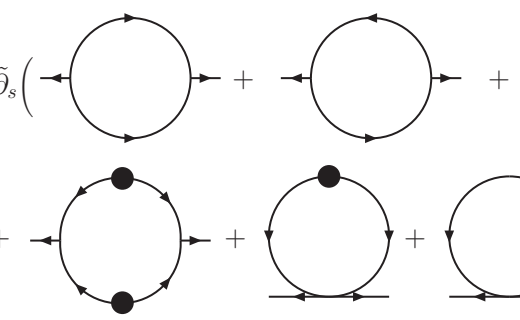

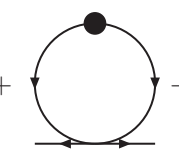

(e)

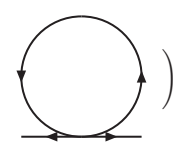

$(f)$
FIG. 5. Diagrammatic representation of the exact flow equation of $\Gamma_{\perp}^{(0,2)}(\nu, \vec{p})$, with $\tilde{\partial}_{s} \equiv \partial_{s} R_{\kappa} \frac{\partial}{\partial R_{\kappa}}+\partial_{s} N_{\kappa} \frac{\partial}{\partial N_{\kappa}}$. The combinatorial factors are not explicitly written, and diagrams involving $\Gamma_{\kappa}^{(n, 0)}$ vertices are omitted since they are vanishing.

frequency. The flow equations for the 2-point functions can indeed be exactly closed in this limit using Ward identities [114].

\section{A. Derivation of the flow equations in the large wave-number limit}

The exact flow equations for the 2-point functions (19) are represented diagrammatically in Figs. 4 and 5 , The diagrams involving $\Gamma_{\kappa}^{(n, 0)}$ vertices are not depicted since they are vanishing as a consequence of the general properties of NPRG within the Janssen-de Dominicis formalism and Itō's discretisation [90, 101].
Let us show that the remaining diagrams in Figs. 4 and 5] are either negligible, or closed (expressed in terms of 2-point functions) in the large $|\vec{p}|$ limit. Indeed, the presence of the regulator term $\partial_{\kappa} \mathcal{R}_{\kappa}$ (implicit in the $\tilde{\partial}_{s}$ operator) effectively cuts off the internal wave-vector $\vec{q}$ integral to values of order $|\vec{q}| \lesssim \kappa$ (and similar conclusions can be drawn when $\vec{p}+\vec{q}$ is cut off instead of $\vec{q}$, see Appendix F). In the limit of large external wave-number $|\vec{p}| \gg \kappa$, the internal wave-vector $\vec{q}$ is negligible in all vertices compared to $\vec{p}$ and can be set to zero. Let us emphasize that this can be safely done since analyticity is ensured for all vertex functions at any scale $\kappa \neq 0$ [115], and only $|\vec{p}| \gg|\vec{q}|$ is required, without assumptions on $\kappa$. This implies that, in this limit, the 3 - and 4-point vertex functions are to be evaluated at one, respectively two, vanishing wave-vectors, and this implies

- if the zero wave-vector is carried by a $\overline{\vec{u}}$-leg, then the corresponding vertex is vanishing as a consequence of the time-gauged shift symmetry (encoded in the general Ward identity (E9) and thus such diagrams are negligible in the large $\vec{p}$ limit (diagrams (a), (b), (d) in Fig. 4 and (a), (b), (f) in Fig. 5).

- if the zero wave-vector is carried by a $\vec{u}$-leg, then the corresponding vertex is exactly related to lower order vertices by time-gauged Galilean Ward identities and thus such diagrams are closed in the large $\vec{p}$ limit.

Let us make this last assertion explicit. There are two 3 -point vertex functions, $\Gamma^{(2,1)}$ and $\Gamma^{(1,2)}$, involved in the (non-zero) diagrams (c) of Fig. 4 and (c) and (d) of Fig. 5, which are to be evaluated at a zero wave-vector on a $\vec{u}$-leg. As derived in Appendix E, they are related by a time-gauged Galilean Ward identity to 2-point vertex functions as

$$
\begin{aligned}
& \Gamma_{\alpha \beta \gamma}^{(2,1)}(\nu, \overrightarrow{0} ; \omega, \vec{q})=-\frac{q^{\alpha}}{\nu}\left(\Gamma_{\beta \gamma}^{(1,1)}(\nu+\omega, \vec{q})-\Gamma_{\beta \gamma}^{(1,1)}(\omega, \vec{q})\right) \\
& \Gamma_{\alpha \beta \gamma}^{(1,2)}(\nu, \overrightarrow{0} ; \omega, \vec{q})=-\frac{q^{\alpha}}{\nu}\left(\Gamma_{\beta \gamma}^{(0,2)}(\nu+\omega, \vec{q})-\Gamma_{\beta \gamma}^{(0,2)}(\omega, \vec{q})\right) .
\end{aligned}
$$

Similarly, one can deduce from the Ward identities (E19), (E20), (85) and (86) that the two 4-point vertex functions, $\Gamma^{(2,2)}$ and $\Gamma^{(3,1)}$, involved in the remaining diagrams (e) of Figs. 4 and 5 , to be evaluated at two vanishing wave-vectors, are related to 2-point vertex functions as 


$$
\begin{aligned}
& \Gamma_{\alpha \beta \gamma \delta}^{(2,2)}(\omega, \vec{q}=\overrightarrow{0},-\omega,-\vec{q}=\overrightarrow{0}, \nu, \vec{p})=\frac{p^{\alpha} p^{\beta}}{\omega^{2}}\left[\Gamma_{\gamma \delta}^{(0,2)}(\omega+\nu, \vec{p})-2 \Gamma_{\gamma \delta}^{(0,2)}(\nu, \vec{p})+\Gamma_{\gamma \delta}^{(0,2)}(-\omega+\nu, \vec{p})\right] \\
& \Gamma_{\alpha \beta \gamma \delta}^{(3,1)}(\omega, \vec{q}=\overrightarrow{0},-\omega,-\vec{q}=\overrightarrow{0}, \nu, \vec{p})=\frac{p^{\alpha} p^{\beta}}{\omega^{2}}\left[\Gamma_{\gamma \delta}^{(1,1)}(\omega+\nu, \vec{p})-2 \Gamma_{\gamma \delta}^{(1,1)}(\nu, \vec{p})+\Gamma_{\gamma \delta}^{(1,1)}(-\omega+\nu, \vec{p})\right] .
\end{aligned}
$$

Thus, the expressions of all the non-vanishing diagrams contributing to the flows of the 2-point functions can be exactly closed, i.e. expressed in terms of 2-point functions only, in the limit of large external wave-number.

Let us emphasize that, since the Galilean symmetry is gauged in time, no approximation is needed for the internal frequency once the internal wave-vector is neglected. This is a great advantage since an expansion on the internal frequency would not be justified as it is not cut off. Indeed, the regulator functions $N_{\kappa}$ and $R_{\kappa}$ only depend on momenta, but not on frequencies (which is re- quired in order to maintain the various symmetries of the model along the flow). Hence, the internal momentum can safely be neglected when $|\vec{p}| \gg \kappa$, and once this is done, the internal frequency dependence is entirely fixed by the symmetries and no approximation is necessary in the frequency sector.

The non-zero diagrams (c), (e) of Fig. 团and (c), (d), (e) of Fig. 5 are explicitly calculated in Appendix F. Gathering their contributions, the NPRG evolution of the transverse 2-point functions in the limit $|\vec{p}| \gg \kappa$ is given by the exact flow equations

$$
\begin{aligned}
\partial_{s} \Gamma_{\perp}^{(1,1)}(\nu, \vec{p}) & =\frac{(d-1)}{d} p^{2} \int_{\omega}\left\{-\left[\frac{\Gamma_{\perp}^{(1,1)}(\omega+\nu, \vec{p})-\Gamma_{\perp}^{(1,1)}(\nu, \vec{p})}{\omega}\right]^{2} G_{\perp}^{u \bar{u}}(-\omega-\nu, \vec{p})\right. \\
& \left.+\frac{1}{2 \omega^{2}}\left[\Gamma_{\perp}^{(1,1)}(\omega+\nu, \vec{p})-2 \Gamma_{\perp}^{(1,1)}(\nu, \vec{p})+\Gamma_{\perp}^{(1,1)}(-\omega+\nu, \vec{p})\right]\right\} \times \tilde{\partial}_{s} \int_{\vec{q}} G_{\perp}^{u u}(\omega, \vec{q}) \\
\partial_{s} \Gamma_{\perp}^{(0,2)}(\nu, \vec{p}) & =\frac{(d-1)}{d} p^{2} \int_{\omega}\left\{\left|\frac{\Gamma_{\perp}^{(1,1)}(\nu, \vec{p})-\Gamma_{\perp}^{(1,1)}(\nu+\omega, \vec{p})}{\omega}\right|^{2} G_{\perp}^{u u}(\omega+\nu, \vec{p})\right. \\
& -2\left[\frac{\Gamma_{\perp}^{(0,2)}(\omega+\nu, \vec{p})-\Gamma_{\perp}^{(0,2)}(\nu, \vec{p})}{\omega}\right] \times \Re\left\{\left[\frac{\Gamma_{\perp}^{(1,1)}(\omega+\nu, \vec{p})-\Gamma_{\perp}^{(1,1)}(\nu, \vec{p})}{\omega}\right] G_{\perp}^{u \bar{u}}(-\omega-\nu, \vec{p})\right\} \\
& \left.+\frac{1}{2 \omega^{2}}\left[\Gamma_{\perp}^{(0,2)}(\omega+\nu, \vec{p})-2 \Gamma_{\perp}^{(0,2)}(\nu, \vec{p})+\Gamma_{\perp}^{(0,2)}(-\omega+\nu, \vec{p})\right]\right\} \times \tilde{\partial}_{s} \int_{\vec{q}} G_{\perp}^{u u}(\omega, \vec{q}) .
\end{aligned}
$$

We study below the decoupling property of these equations and its consequences.

\section{B. Study of the (non-)decoupling}

In this section, we prove that the large wave-number sector does not decouple in the NS flow equations Eqs. (89) and (90). Our strategy is as previously to assume that such a decoupling does take place and then to show that this leads to a contradiction.

We consider the inertial regime of wave-numbers $|\vec{p}|$ much larger than the running inverse integral scale $\kappa$ and much smaller than the inverse microscopic Kolmogorov scale. The effective action $\Gamma_{\kappa}$ has thus already approached the IR attractive fixed point, and it is conve- nient to rewrite Eqs. (89) and (90) in terms of dimensionless quantities:

$$
\begin{aligned}
& \partial_{s} \Gamma_{\perp}^{(1,1)}(\nu, p)=\kappa^{2} \nu_{\kappa}\left\{\left(2-\eta^{\nu}\right) \hat{\Gamma}_{\perp}^{(1,1)}(\hat{\nu}, \hat{p})-\hat{p} \partial_{\hat{p}} \hat{\Gamma}_{\perp}^{(1,1)}(\hat{\nu}, \hat{p})\right. \\
& \left.\quad-\left(2-\eta^{\nu}\right) \hat{\nu} \partial_{\hat{\nu}} \hat{\Gamma}_{\perp}^{(1,1)}(\hat{\nu}, \hat{p})+\partial_{s} \hat{\Gamma}_{\perp}^{(1,1)}(\hat{\nu}, \hat{p})\right\} \\
& \partial_{s} \Gamma_{\perp}^{(0,2)}(\nu, p)=D_{\kappa}\left\{-\eta^{D} \hat{\Gamma}_{\perp}^{(0,2)}(\hat{\nu}, \hat{p})-\hat{p} \partial_{\hat{p}} \hat{\Gamma}_{\perp}^{(0,2)}(\hat{\nu}, \hat{p})\right. \\
& \left.\quad-\left(2-\eta^{\nu}\right) \hat{\nu} \partial_{\hat{\nu}} \hat{\Gamma}_{\perp}^{(0,2)}(\hat{\nu}, \hat{p})+\partial_{s} \hat{\Gamma}_{\perp}^{(0,2)}(\hat{\nu}, \hat{p})\right\}
\end{aligned}
$$

where

$$
\begin{aligned}
& \Gamma_{\perp}^{(1,1)}(\nu, p)=\kappa^{2} \nu_{\kappa} \hat{\Gamma}_{\perp}^{(1,1)}(\hat{\nu}, \hat{p}) \\
& \Gamma_{\perp}^{(0,2)}(\nu, p)=D_{\kappa} \hat{\Gamma}_{\perp}^{(0,2)}(\hat{\nu}, \hat{p}) .
\end{aligned}
$$


At the fixed point, by definition $\partial_{s} \hat{\Gamma}_{\perp}^{(1,1)}(\hat{\nu}, \hat{p})=$ $\partial_{s} \hat{\Gamma}_{\perp}^{(0,2)}(\hat{\nu}, \hat{p})=0$ and the running coefficients behave as $D_{\kappa} \sim \kappa^{-\eta^{D}}$ and $\nu_{\kappa} \sim \kappa^{-\eta^{\nu}}$. We now assume that for $|\vec{p}| \gg \kappa$, the right hand sides of Eqs. (89) and (90) are negligible (decoupled), that is $\partial_{s} \Gamma_{\perp}^{(0,2)} \simeq \partial_{s} \Gamma_{\perp}^{(1,1)} \simeq 0$ in Eqs. (91). The general solutions of the remnant homogeneous linear equations are the scaling forms

$$
\begin{aligned}
& \hat{\Gamma}_{\perp}^{(1,1)}(\hat{\nu}, \hat{p})=\hat{p}^{2-\eta^{\nu}} \hat{\chi}^{(1,1)}\left(\hat{\nu} / \hat{p}^{2-\eta^{\nu}}\right), \\
& \hat{\Gamma}_{\perp}^{(0,2)}(\hat{\nu}, \hat{p})=\hat{p}^{-\eta^{D}} \hat{\chi}^{(0,2)}\left(\hat{\nu} / \hat{p}^{2-\eta^{\nu}}\right),
\end{aligned}
$$

or, equivalently,

$$
\begin{aligned}
& \Gamma_{\perp}^{(1,1)}(\nu, p)=p^{2-\eta^{\nu}} \chi^{(1,1)}\left(\nu / p^{2-\eta^{\nu}}\right), \\
& \Gamma_{\perp}^{(0,2)}(\nu, p)=p^{-\eta^{D}} \chi^{(0,2)}\left(\nu / p^{2-\eta^{\nu}}\right) .
\end{aligned}
$$

Both functions $\chi^{(i, j)}(z)$ and $\hat{\chi}^{(i, j)}(z)$ are equal up to some (non-universal) normalisations (of the functions and of their arguments).

Let us now prove that this is inconsistent. For this, we substitute the obtained solutions (94) in the right hand sides of Eqs. (89) and (90) and show that they are not negligible compared to the other terms of Eqs. (911). To determine the behavior of the right hand sides of Eqs. (89) and (90), we assign to each quantity appropriate powers of $p$ :

- the internal wave-vector $\vec{q}$ is tailored to values $q \sim \kappa$ by the presence of the term $\partial_{s} \mathcal{R}_{\kappa}$, that is, it is of order one as $p \gg \kappa$.

- the external frequency $\nu$ scales as $p^{2-\eta^{\nu}}$.

- the internal frequency $\omega$ satisfies $\omega \ll \nu \sim p^{2-\eta^{\nu}}$, as shown below.

As a matter of fact, the internal frequency is not cut off by the regulator and it is not clear a priori which region of integration on $\omega$ dominates. There are essentially two scales, $\kappa$ and $p$. If one assumes that $\omega \sim \nu \sim p^{2-\eta^{\nu}}$ then the resulting integral on $\omega$ behaves for small $\omega$ as $\int d \omega / \omega^{2}$ which is IR divergent. This means that the dominating internal frequencies are $\omega \ll \nu \sim p^{2-\eta^{\nu}}$. In this limit, the flow equations (89) and (90) acquire a simpler form

$$
\begin{aligned}
& \partial_{s} \Gamma_{\perp}^{(1,1)}(\nu, \vec{p})=\frac{(d-1)}{d} p^{2} I_{0}\left\{\frac{1}{2} \partial_{\nu}^{2} \Gamma_{\perp}^{(1,1)}(\nu, \vec{p})\right. \\
&\left.-\left(\partial_{\nu} \Gamma_{\perp}^{(1,1)}(\nu, \vec{p})\right)^{2} G_{\perp}^{u \bar{u}}(-\nu, \vec{p})\right\} \\
& \partial_{s} \Gamma_{\perp}^{(0,2)}(\nu, \vec{p})=\frac{(d-1)}{d} p^{2} I_{0}\left\{\frac{1}{2} \partial_{\nu}^{2} \Gamma_{\perp}^{(0,2)}(\nu, \vec{p})\right. \\
&- 2 \partial_{\nu} \Gamma_{\perp}^{(0,2)}(\nu, \vec{p}) \times \Re\left[\partial_{\nu} \Gamma_{\perp}^{(1,1)}(\nu, \vec{p}) G_{\perp}^{u \bar{u}}(-\nu, \vec{p})\right] \\
&\left.+\left|\partial_{\nu} \Gamma_{\perp}^{(1,1)}(\nu, \vec{p})\right|^{2} G_{\perp}^{u u}(\nu, \vec{p})\right\}
\end{aligned}
$$

where

$$
I_{0}=\tilde{\partial}_{s} \int_{\omega, \vec{q}} G_{\perp}^{u u}(\omega, \vec{q})
$$

Under this form, the relevant scale for the internal frequency $\omega$ is manifestly $\kappa$ since the integral $I_{0}$ does not depend on the external scales $p$ or $\nu$.

Substituting the scaling solutions (94) in the righthand sides of Eqs. (95) and (96) one obtains that the equations for $\Gamma^{(1,1)}(\nu, p)$ and $\Gamma^{(0,2)}(\nu, p)$ behave as $p^{\eta^{\nu}}$ and $p^{-2-\eta^{D}+2 \eta^{\nu}}$, respectively. Since the left-hand sides behave as $p^{2-\eta^{\nu}}$ and $p^{-\eta^{D}}$, respectively, this yields violations of the scaling which are not marginal, but quite substancial: the right-hand sides are not sub-leading compared to the left-hand ones but dominating by a factor $p^{2 / 3}$ in $d=3$ and $p^{2}$ in $d=2$. This clearly is not consistent and proves that there is no decoupling of the large wave-number sector $|\vec{p}| \gg \kappa$.

\section{Consequences of the non-decoupling and intermittency}

This non-decoupling property is extremely peculiar. It means that correlation functions remain sensitive to the integral scale even at $|\vec{p}| \gg \kappa$, which is completely different from what occurs in critical phenomena, where correlation functions have a well-behaved infinite-volume limit. The prominence of the integral scale in the onset of intermittency was already observed in the perturbative context [19]. The origin of this difference can be intuitively understood. A dissipative system such as a fluid cannot sustain well-defined stationary correlation functions without injection of energy, and thus it remains in some way sensitive to the corresponding scale, the integral scale, even at much larger wave-length scales. It is therefore reasonable to infer that this violation of scaling is general in fully developed turbulence, and not restricted to 2-point correlation functions. This could explain the origin of intermittency: correlation functions are dominated by the existence of an IR fixed point, leading to power-law behavior, but the absence of decoupling prevents the existence of usual scaling (determined by a finite set of anomalous dimensions, here $\eta^{\nu}$ and $\eta^{D}$ ), and opens the door to multiscaling.

Let us make one further step and try to explain why the lowest order structure functions display only very small corrections to the dimensional scaling exponents. Obviously the four-fifth theorem forbids anomalous corrections for the $S^{(3)}$ structure function. As for $S^{(2)}$, one can justify very small but non-zero corrections in the following way. In the regime of large wave-numbers, the equations (95) and (96) become exact. These equations read in terms of the connected 2-point correlation functions

$$
\begin{aligned}
& \partial_{s} G_{\perp}^{u \bar{u}}(\nu, \vec{p})=\frac{(d-1)}{2 d} p^{2} I_{0} \partial_{\nu}^{2} G_{\perp}^{u \bar{u}}(\nu, \vec{p}), \\
& \partial_{s} G_{\perp}^{u u}(\nu, \vec{p})=\frac{(d-1)}{2 d} p^{2} I_{0} \partial_{\nu}^{2} G_{\perp}^{u u}(\nu, \vec{p}) .
\end{aligned}
$$


Again, if the associated dimensionless functions approach a fixed point and if decoupling is assumed, one can show that right-hand sides are enhanced with respect to the other terms by a factor $p^{2 / 3}$ in $d=3$ and $p^{2}$ in $d=2$. However, the functions that are usually measured experimentally are not directly the functions $G_{\perp}^{u \bar{u}}(\nu, \vec{p})$ or $G_{\perp}^{u u}(\nu, \vec{p})$, but correlators at equal times such as

$$
\int \frac{d \omega}{2 \pi} G_{\perp}^{u u}(\omega, \vec{p})
$$

see e.g. Eq. (58). As the function $G_{\perp}^{u u}(\omega, \vec{p})$ is expected to remain regular in frequency when $\kappa \rightarrow 0$, one can integrate Eq. (98) over the frequency, which yields

$$
\partial_{s} \int \frac{d \omega}{2 \pi} G_{\perp}^{u u}(\omega, \vec{p}) \ll \text { leading terms. }
$$

This means that the leading term that violates the decoupling is zero when integrated over frequencies. Accordingly, the possible leading intermittency correction to this quantity comes from a sub-leading contribution. This could explain the smallness of the deviation for the second order structure function. On the contrary, higher-order $n$-point functions bear a more complicated frequency structure and such compensations are very unlikely to occur, and thus intermittency effects could be much larger for higher-order structure functions, as observed in experiments and numerical simulations.

\section{CONCLUSION}

In this paper, we expounded the NPRG formalism to investigate the regime of fully developed isotropic and homogeneous turbulence of the NS equation in the presence of a stochastic forcing. We then developed two complementary approaches to solve the NPRG flow equations. We first implemented a simple approximation, called the LO approximation, which consists in proposing an ansatz for the running effective action $\Gamma_{\kappa}$ based on the NS symmetries. By numerically integrating the corresponding equations, we found a fully attractive fixed point in dimension $d=2$ and $d=3$, governing the stationary regime of fully developed turbulence in the presence of an integral-scale forcing. This fixed point was already identified with approximations similar to ours in $d=3$ in Refs. 84, 86] and in $d=2$ in Ref. 86. The remarkable feature of this fixed point is the emergence of deviations to the dimensional scaling for the two-point functions. These deviations turn out to compensate very precisely for the energy spectrum and the second-order structure function, such that for instance for the energy spectrum, the Kolmogorov scaling $p^{-5 / 3}$ in $d=3$ and the Kraichnan-Batchelor one $p^{-3}$ in $d=2$ (in the direct cascade), are recovered.

To further analyze the regime of large wave-number where these deviations lie, we derived a set of closed flow equations for the 2-point functions, which are exact in this regime. We proved that the usual decoupling property of NPRG flows is violated, that is the large wavenumber sector does not decouple from the flow equations, which in turn prevents the usual scale invariance. More precisely, on the one hand, the existence of the fixed point entails power-law behavior for the correlation functions. On the other hand, the non-decoupling of the large wavenumbers allows for violations of simple scaling to occur, which means that the exponents can deviate from their dimensional values. This opens the door to multiscaling and intermittency. We also suggested why these deviations remain small for the low-order structure functions (and the energy spectrum), but may be larger for higher-order ones. The value of the corresponding intermittency exponents can be computed by integrating the exact flow equations obtained in the large wave-number regime, which will be investigated in a future work. It would also be interesting to work out the link between the absence of operators of negative dimensions in the OPE in the perturbative context and the non-decoupling property unveiled in the NPRG framework. More generally, the purpose of the present work is to provide a detailed basis for future investigation of NS turbulence using NPRG methods.

\section{ACKNOWLEDGMENTS}

The authors acknowledge financial support from the ECOS-Sud France-Uruguay program U11E01, and from the PEDECIBA. LC and BD thank the Universidad de la República (Uruguay) for hospitality during the completion of this work, and NW the LPTMC for hospitality during his sabbatical year 2012-2013.

\section{APPENDIX A: GENERAL STRUCTURE OF THE NPRG PROPAGATOR}

In this Appendix, we establish the general structure of the $4 \times 4$ propagator matrix $G_{\kappa}$ defined as the inverse of $\left[\Gamma_{\kappa}^{(2)}+\mathcal{R}_{\kappa}\right]$. The matrix elements of $\left[\Gamma_{\kappa}^{(2)}+\mathcal{R}_{\kappa}\right]$ are obtained by taking functional derivatives of (27) and (10) with respect to two of the fields $u_{\alpha}, \bar{u}_{\alpha}, p$ and $\bar{p}$. They are given in Fourier space and at zero fields by, (omitting the $\kappa$ indices to alleviate notation) 


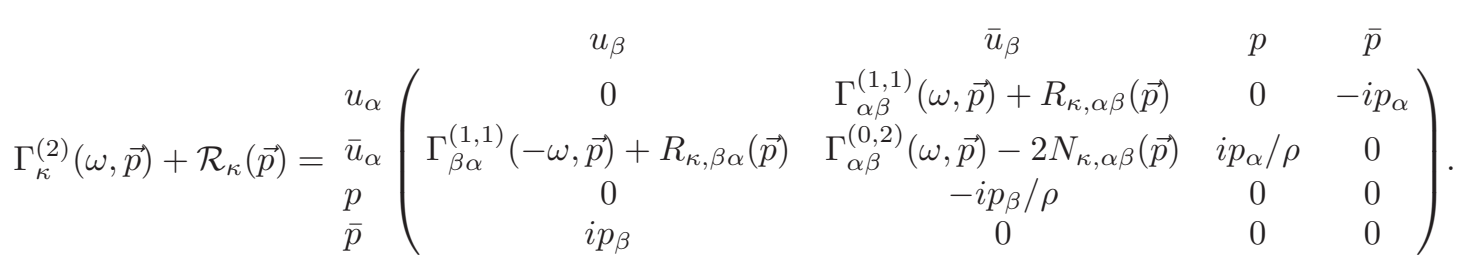

Using rotational invariance and parity, one may infer that the propagator matrix is endowed with the following generic structure

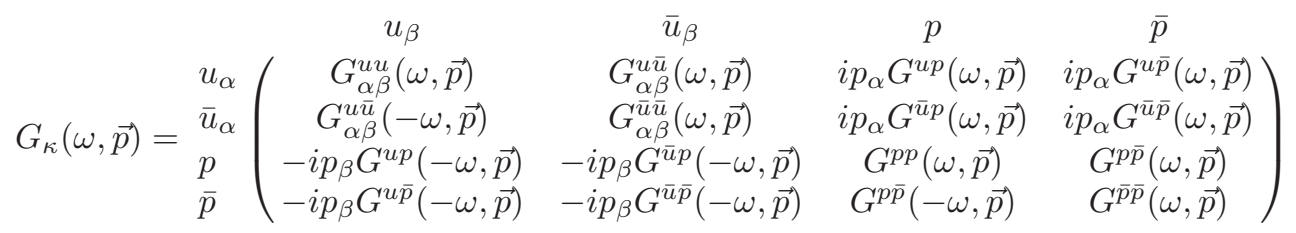

in obvious notation for the two upper indices of the different matrix elements of $G_{\kappa}$. The latters are obtained by requiring that the product $\left[\Gamma_{\kappa}^{(2)}+\mathcal{R}_{\kappa}\right](\omega, \vec{p}) G_{\kappa}(\omega, \vec{p})$ is the identity matrix. This yields in the pressure sector

$$
\begin{aligned}
G^{p p}(\omega, \vec{p}) & =\frac{2 \rho}{p^{2}} f_{\|}^{D}(\vec{p}) \\
G^{p \bar{p}}(\omega, \vec{p}) & =\frac{\rho}{p^{2}}\left(-i \omega+f_{\|}^{\nu}(\vec{p})\right) \\
G^{\bar{p}}(\omega, \vec{p}) & =0 .
\end{aligned}
$$

In the mixed sector, only two elements are non-vanishing, which are

$$
G^{u \bar{p}}(\omega, \vec{p})=-\frac{1}{p^{2}}, \quad G^{\bar{u} p}(\omega, \vec{p})=\frac{\rho}{p^{2}} .
$$

As for the velocity sector, one obtains that all longitudinal components vanish. As a consequence, the propagator in this sector is purely transverse and given by

$$
\begin{aligned}
& G_{\alpha \beta}^{u \bar{u}}(\omega, \vec{q})=P_{\alpha \beta}^{\perp}(\vec{q}) \frac{1}{\Gamma_{\perp}^{(1,1)}(-\omega, \vec{q})+R_{\kappa}(\vec{q})} \\
& G_{\alpha \beta}^{u u}(\omega, \vec{q})=-P_{\alpha \beta}^{\perp}(\vec{q}) \frac{\Gamma_{\perp}^{(0,2)}(\omega, \vec{q})-2 N_{\kappa}(\vec{q})}{\left|\Gamma_{\perp}^{(1,1)}(\omega, \vec{q})+R_{\kappa}(\vec{q})\right|^{2}} \\
& G_{\alpha \beta}^{\bar{u} \bar{u}}(\omega, \vec{q})=0 .
\end{aligned}
$$

\section{APPENDIX B: DERIVATION OF THE NPRG FLOW EQUATIONS AT LO}

In this Appendix, we derive the flow equations for the transverse components of the two running functions $f_{\alpha \beta}^{\nu}(\vec{p})$ and $f_{\alpha \beta}^{D}(\vec{p})$ of the LO ansatz. They are related to the flows of $\Gamma_{\alpha \beta}^{(1,1)}(\nu=0, \vec{p})$ and $\Gamma_{\alpha \beta}^{(0,2)}(\nu=0, \vec{p})$, respectively, which we now calculate.

\section{Flow equations of $\Gamma_{\alpha \beta}^{(1,1)}$ and $\Gamma_{\alpha \beta}^{(0,2)}$ at $\mathbf{L O}$}

According to Eq. (19), the flow equation of $\Gamma_{\alpha \beta}^{(1,1)}(\nu=0, \vec{p})$ is given by

$$
\partial_{\kappa} \Gamma_{\alpha \beta}^{(1,1)}(\nu=0, \vec{p})=\operatorname{Tr} \int_{\omega, \vec{q}} \partial_{\kappa} \mathcal{R}_{\kappa}(\vec{q}) \cdot G_{\kappa}(\mathbf{q}) \cdot\left\{\Gamma_{\kappa, u_{\alpha}}^{(3)}(\mathbf{p}, \mathbf{q}) \cdot G_{\kappa}(\mathbf{p}+\mathbf{q}) \cdot \Gamma_{\kappa, \bar{u}_{\beta}}^{(3)}(\mathbf{p}+\mathbf{q},-\mathbf{p})\right\} \cdot G_{\kappa}(\mathbf{q})
$$

omitting the contributions of the 4-point vertices which are vanishing at LO. As apparent in (A1), the regulator matrix $\mathcal{R}_{\kappa}$ has only three non-vanishing entries: $\left[\mathcal{R}_{\kappa}\right]_{12}=\left[\mathcal{R}_{\kappa}\right]_{21}=R_{\kappa}(\vec{p})$ and $\left[\mathcal{R}_{\kappa}\right]_{12}=-2 N_{\kappa}(\vec{p})$. Moreover, at LO, only the vertex function $\Gamma_{\alpha \beta \gamma}^{(2,1)}$ is non-zero and contributes in the $\Gamma_{\kappa, i}^{(3)}$ matrices. Performing the matrix product (B1) 
and taking the trace, one is left with only four terms, which are

$$
\begin{aligned}
\partial_{\kappa} \Gamma_{\alpha \beta}^{(1,1)}(\nu=0, \vec{p}) & =\lambda^{2} \int_{\omega, \vec{q}} \partial_{\kappa} R_{\kappa}(\vec{q})\left\{i\left(q_{l} \delta_{i \beta}-(p+q)_{i} \delta_{l \beta}\right) i\left((p+q)_{\alpha} \delta_{k j}-p_{k} \delta_{\alpha j}\right) \frac{P_{i j}^{\perp}(\vec{q}) P_{k l}^{\perp}(\vec{p}+\vec{q})}{\left(-i \omega+\tilde{f}_{\perp}^{\nu}(\vec{q})\right)^{2}} \frac{2 \tilde{f}_{\perp}^{D}(\vec{p}+\vec{q})}{\left(\omega^{2}+\left(\tilde{f}_{\perp}^{\nu}(\vec{p}+\vec{q})\right)^{2}\right)}\right\} \\
& +\lambda^{2} \int_{\omega, \vec{q}}\left\{\partial_{\kappa} R_{\kappa}(\vec{q}) \frac{4 \tilde{f}_{\perp}^{D}(\vec{q}) \tilde{f}_{\perp}^{\nu}(\vec{q})}{\left(\omega^{2}+\left(\tilde{f}_{\perp}^{\nu}(\vec{q})\right)^{2}\right)^{2}}-2 \frac{\partial_{\kappa} N_{\kappa}(\vec{q})}{\omega^{2}+\left(\tilde{f}_{\perp}^{\nu}(\vec{q})\right)^{2}}\right\} \\
& \times\left\{i\left(-q_{l} \delta_{i \beta}+(q+p)_{i} \delta_{\beta l}\right) i\left(q_{\alpha} \delta_{j k}+p_{j} \delta_{\alpha k}\right) \frac{P_{i j}^{\perp}(\vec{q}) P_{k l}^{\perp}(\vec{p}+\vec{q})}{i \omega+\tilde{f}_{\perp}^{\nu}(\vec{p}+\vec{q})}\right\}
\end{aligned}
$$

with the notation

$$
\tilde{f}_{\perp}^{\nu}(\vec{q})=f_{\perp}^{\nu}(\vec{q})+R_{\kappa}(\vec{q}) \quad \text { and } \quad \tilde{f}_{\perp}^{D}(\vec{q})=f_{\perp}^{D}(\vec{q})+N_{\kappa}(\vec{q}) .
$$

This flow equation is to be projected onto the transverse sector. Hence, all the terms proportional to $p_{\alpha}$ or $p_{\beta}$ can be discarded since $P_{\alpha \beta}^{\perp}(\vec{p}) p_{\alpha}=P_{\alpha \beta}^{\perp}(\vec{p}) p_{\beta}=0$. The two tensor structures in Eq. (B2) can be then simplified in the following way

$$
\begin{aligned}
T_{a}^{(1,1)} & \equiv-P_{i j}^{\perp}(\vec{q})\left((p+q)_{\alpha} \delta_{k j}-p_{k} \delta_{\alpha j}\right) P_{k l}^{\perp}(\vec{p}+\vec{q})\left(q_{l} \delta_{i \beta}-(p+q)_{i} \delta_{l \beta}\right) \\
& =\left[-\vec{p}^{2}+\frac{(\vec{p} \cdot(\vec{p}+\vec{q}))^{2}}{(\vec{p}+\vec{q})^{2}}\right] \delta_{\alpha \beta}-2 \frac{q_{\alpha} q_{\beta}}{\vec{q}^{2}} \vec{p} \cdot \vec{q}+\quad \text { longitudinal parts } \\
T_{b}^{(1,1)} & \equiv-P_{i j}^{\perp}(\vec{q})\left(q_{\alpha} \delta_{k j}+p_{j} \delta_{\alpha k}\right) P_{k l}^{\perp}(\vec{p}+\vec{q})\left(-q_{l} \delta_{i \beta}+(p+q)_{i} \delta_{l \beta}\right) \\
& =\left[-\vec{p}^{2}+\frac{(\vec{p} \cdot \vec{q}))^{2}}{\vec{q}^{2}}\right] \delta_{\alpha \beta}+2 \frac{q_{\alpha} q_{\beta}}{(\vec{q}+\vec{p})^{2}} \vec{p} \cdot(\vec{p}+\vec{q}) \quad+\quad \text { longitudinal parts }
\end{aligned}
$$

where only the transverse contributions are explicitly specified.

Similarly, the flow equation of $\Gamma_{\alpha \beta}^{(0,2)}(\nu=0, \vec{p})$ is given by Eq. (19)

$$
\partial_{\kappa} \Gamma_{\alpha \beta}^{(0,2)}(\nu=0, \vec{p})=\operatorname{Tr} \int_{\omega, \vec{q}} \partial_{\kappa} \mathcal{R}_{\kappa}(\vec{q}) \cdot G_{\kappa}(\mathbf{q}) \cdot\left\{\Gamma_{\kappa, \bar{u}_{\alpha}}^{(3)}(\mathbf{p}, \mathbf{q}) \cdot G_{\kappa}(\mathbf{p}+\mathbf{q}) \cdot \Gamma_{\kappa, \bar{u}_{\beta}}^{(3)}(\mathbf{p}+\mathbf{q},-\mathbf{p})\right\} \cdot G_{\kappa}(\mathbf{q})
$$

omitting the vanishing 4-point vertices (at LO). Only three terms are left in the trace of the matrix product (B5), which are

$$
\begin{aligned}
\partial_{\kappa} \Gamma_{\alpha \beta}^{(0,2)}(\nu=0, \vec{p}) & =\lambda^{2} \int_{\omega, \vec{q}} i\left((q+p)_{i} \delta_{j \alpha}-q_{j} \delta_{\alpha i}\right) i\left(q_{k} \delta_{l \beta}-(p+q)_{l} \delta_{\beta k}\right) P_{i l}^{\perp}(\vec{q}) P_{k j}^{\perp}(\vec{p}+\vec{q}) \\
& \times\left\{\partial_{\kappa} R_{\kappa}(\vec{q}) \frac{4 \tilde{f}_{\perp}^{D}(\vec{q}) \tilde{f}_{\perp}^{\nu}(\vec{q})}{\left(\omega^{2}+\left(\tilde{f}_{\perp}^{\nu}(\vec{q})\right)^{2}\right)^{2}}-2 \frac{\partial_{\kappa} N_{\kappa}(\vec{q})}{\omega^{2}+\left(\tilde{f}_{\perp}^{\nu}(\vec{q})\right)^{2}}\right\} \frac{2 \tilde{f}_{\perp}^{D}(\vec{q}+\vec{p})}{\omega^{2}+\left(\tilde{f}_{\perp}^{\nu}(\vec{p}+\vec{q})\right)^{2}} .
\end{aligned}
$$

As previously, ignoring the terms proportional to $p_{\alpha}$ and $p_{\beta}$, the tensor structure in this equation simplifies to

$$
\begin{aligned}
T^{(0,2)} & =-P_{i l}^{\perp}(\vec{q})\left(p_{i} \delta_{j \alpha}+p_{j} \delta_{i \alpha}\right) P_{j k}^{\perp}(\vec{p}+\vec{q})\left(-p_{l} \delta_{k \beta}-p_{k} \delta_{l \beta}\right) \quad+\quad \text { longitudinal parts } \\
& =\left[2 \vec{p}^{2}+\frac{(\vec{p} \cdot(\vec{p}+\vec{q}))^{2}}{(\vec{p}+\vec{q})^{2}}-\frac{(\vec{p} \cdot \vec{q})^{2}}{\vec{q}^{2}}\right] \delta_{\alpha \beta}+2 \frac{q_{\alpha} q_{\beta}}{\vec{q}^{2}(\vec{p}+\vec{q})^{2}}\left(\vec{p} \vec{p}^{2} \cdot \vec{q}+2(\vec{p} \cdot \vec{q})^{2}-\vec{p}^{2} \vec{q}^{2}\right) \quad+\quad \text { longitudinal parts. }
\end{aligned}
$$

\section{Flow equations of $f_{\perp}^{D}$ and $f_{\perp}^{\nu}$}

According to the expressions (63) of the 2-point functions at LO in the velocity sector, the flow equations of the transverse functions $f_{\perp}^{D}$ and $f_{\perp}^{\nu}$ may be defined as

$$
\begin{aligned}
& \partial_{\kappa} f_{\perp}^{\nu}(\vec{p})=\frac{1}{(d-1)} P_{\alpha \beta}^{\perp}(\vec{p}) \partial_{\kappa} \Gamma_{\alpha \beta}^{(1,1)}(\nu=0, \vec{p}) \\
& \partial_{\kappa} f_{\perp}^{D}(\vec{p})=-\frac{1}{2(d-1)} P_{\alpha \beta}^{\perp}(\vec{p}) \partial_{\kappa} \Gamma_{\alpha \beta}^{(0,2)}(\nu=0, \vec{p}) .
\end{aligned}
$$


The flow equations of $\partial_{\kappa} \Gamma_{\alpha \beta}^{(1,1)}$ and $\partial_{\kappa} \Gamma_{\alpha \beta}^{(0,2)}$ are proportional to the tensor structures (B4) and (B7), respectively, which projections onto the transverse sector are straightforward, using

$$
P_{\alpha \beta}^{\perp}(\vec{p}) \delta_{\alpha \beta}=d-1 \quad \text { and } \quad P_{\alpha \beta}^{\perp}(\vec{p}) q_{\alpha} q_{\beta}=q^{2}-\frac{(\vec{p} \cdot \vec{q})^{2}}{\vec{p}^{2}} .
$$

One obtains

$$
\begin{aligned}
\partial_{\kappa} f_{\perp}^{\nu}(\vec{p}) & =\frac{\lambda^{2}}{(d-1)} \int_{\omega, \vec{q}}\left\{\frac{2 \tilde{f}_{\perp}^{D}(\vec{p}+\vec{q}) \partial_{\kappa} R_{\kappa}(\vec{q})}{\left(-i \omega+\tilde{f}_{\perp}^{\nu}(\vec{q})\right)^{2}\left(\omega^{2}+\left(\tilde{f}_{\perp}^{\nu}(\vec{p}+\vec{q})\right)^{2}\right)}\left[\left(-\vec{p}^{2}+\frac{(\vec{p} \cdot(\vec{p}+\vec{q}))^{2}}{(\vec{p}+\vec{q})^{2}}\right)(d-1)-2 \vec{p} \cdot \vec{q}\left(1-\frac{(\vec{p} \cdot \vec{q})^{2}}{\vec{q}^{2} \vec{p}^{2}}\right)\right]\right. \\
& +\left\{\partial_{\kappa} R_{\kappa}(\vec{q}) \frac{4 \tilde{f}_{\perp}^{D}(\vec{q}) \tilde{f}_{\perp}^{\nu}(\vec{q})}{\left(\omega^{2}+\left(\tilde{f}_{\perp}^{\nu}(\vec{q})\right)^{2}\right)^{2}}-2 \frac{\partial_{\kappa} N_{\kappa}(\vec{q})}{\omega^{2}+\left(\tilde{f}_{\perp}^{\nu}(\vec{q})\right)^{2}}\right\} \frac{1}{i \omega+\tilde{f}_{\perp}^{\nu}(\vec{p}+\vec{q})} \\
& \left.\times\left[\left(-\vec{p}^{2}+\frac{(\vec{p} \cdot \vec{q})^{2}}{\vec{q}^{2}}\right)(d-1)+2 \frac{\vec{p} \cdot(\vec{p}+\vec{q})}{(\vec{q}+\vec{p})^{2}}\left(\vec{q}^{2}-\frac{(\vec{p} \cdot \vec{q})^{2}}{\vec{p}^{2}}\right)\right]\right\} \\
\partial_{\kappa} f_{\perp}^{D}(\vec{p}) & =-\frac{\lambda^{2}}{2(d-1)} \int_{\omega, \vec{q}}\left\{\left[\partial_{\kappa} R_{\kappa}(\vec{q}) \frac{4 \tilde{f}_{\perp}^{D}(\vec{q}) \tilde{f}_{\perp}^{\nu}(\vec{q})}{\left(\omega^{2}+\left(\tilde{f}_{\perp}^{\nu}(\vec{q})\right)^{2}\right)^{2}}-2 \frac{\partial_{\kappa} N_{\kappa}(\vec{q})}{\omega^{2}+\left(\tilde{f}_{\perp}^{\nu}(\vec{q})\right)^{2}}\right] \frac{2 \tilde{f}_{\perp}^{D}(\vec{q}+\vec{p})}{\omega^{2}+\left(\tilde{f}_{\perp}^{\nu}(\vec{p}+\vec{q})\right)^{2}}\right. \\
& \left.\times\left[\left(2 \vec{p}^{2}+\frac{(\vec{p} \cdot(\vec{p}+\vec{q}))^{2}}{(\vec{p}+\vec{q})^{2}}-\frac{(\vec{p} \cdot \vec{q})^{2}}{\vec{q}^{2}}\right)(d-1)+2 \frac{1}{\vec{q}^{2}\left(\vec{p}+\vec{q}^{2}\right.}\left(\vec{q}^{2}-\frac{(\vec{p} \cdot \vec{q})^{2}}{\vec{p}^{2}}\right)\left(\vec{p}^{2} \vec{p} \cdot \vec{q}+2(\vec{p} \cdot \vec{q})^{2}-\vec{p}^{2} \vec{q}^{2}\right)\right]\right\} .
\end{aligned}
$$

Within the LO approximation, the frequency dependence remains the bare one, and the integration over the internal frequency $\omega$ can be carried out analytically in the above expressions. Denoting $A=\tilde{f}_{\perp}^{\nu}(\vec{q})^{2}$ and $B=\tilde{f}_{\perp}^{\nu}(\vec{p}+\vec{q})^{2}$, the different frequency integrals are given by

$$
\begin{aligned}
& I_{1}=\int \frac{d \omega}{2 \pi} \frac{1}{(-i \omega+A)^{2}} \frac{1}{\omega^{2}+B^{2}}=\frac{1}{2 B(A+B)^{2}} \\
& I_{2}=\int \frac{d \omega}{2 \pi} \frac{1}{i \omega+B^{2}} \frac{1}{\omega^{2}+A^{2}}=\frac{1}{2 A(A+B)} \\
& I_{3}=\int \frac{d \omega}{2 \pi} \frac{1}{i \omega+B} \frac{1}{\left(\omega^{2}+A^{2}\right)^{2}}=\frac{2 A+B}{4 A^{3}(A+B)^{2}} \\
& I_{4}=\int \frac{d \omega}{2 \pi} \frac{1}{\omega^{2}+B^{2}} \frac{1}{\omega^{2}+A^{2}}=\frac{1}{2 A B(A+B)} \\
& I_{5}=\int \frac{d \omega}{2 \pi} \frac{1}{\omega^{2}+B^{2}} \frac{1}{\left(\omega^{2}+A^{2}\right)^{2}}=\frac{2 A+B}{4 A^{3} B(A+B)^{2}}
\end{aligned}
$$

which yields the two flow equations (67) and (68).

\section{APPENDIX C: AVERAGE INJECTED POWER PER UNIT MASS}

The injected power per unit mass is $f_{\alpha}(\mathbf{x}) v_{\alpha}(\mathbf{x})$. The average of a quantity linearly depending on the stochastic forcing $\vec{f}$ can be calculated using the Janssen-de Dominicis procedure (see Ref. [95] for detail), which yields

$$
\left\langle f_{\alpha}(t, \vec{x}) \mathcal{O}[\vec{v}]\right\rangle=2 \int_{\vec{x}^{\prime}} N_{\kappa, \alpha \beta}\left(\left|\vec{x}-\vec{x}^{\prime}\right|\right)\left\langle\bar{v}_{\beta}\left(t, \vec{x}^{\prime}\right) \mathcal{O}[\vec{v}]\right\rangle .
$$

denoting $\kappa$ the inverse integral scale. Averages of quantities linear in $\vec{f}$ are hence related to response functions. In the particular case where $f_{\alpha}$ and $\mathcal{O}$ are defined at equal times, one must carefully consider the Itō's prescription. As a matter of fact, the average of the injected power per unit mass would naively be given, according to Eq. (C1), by

$$
\begin{aligned}
\left\langle f_{\alpha}(t, \vec{x}) v_{\alpha}(t, \vec{x})\right\rangle \stackrel{\text { naive }}{=} & 2 \int_{\vec{x}^{\prime}} N_{\kappa, \alpha \beta}\left(\left|\vec{x}-\vec{x}^{\prime}\right|\right) \\
& \times\left\langle v_{\alpha}(t, \vec{x}) \bar{v}_{\beta}\left(t, \vec{x}^{\prime}\right)\right\rangle,
\end{aligned}
$$

but the response fonction at equal times is zero because of Itō's prescription. However, the precise meaning of equal time must be carefully specified in discrete time. In particular, for the injected power, one should examine the kinetic energy theorem and properly discretize it. The energy at a given space point comes from both direct injection by the external force and transfer from its neighbouring points. We here only seek the variation of the velocity induced by the external force and thus omit 
tranferred power from one point to another. Accordingly, in discrete time, Itō forward discretization is

$$
f_{\alpha}(t, \vec{x})=\partial_{t} v_{\alpha}(t, \vec{x})=\frac{1}{\delta t}\left(v_{\alpha}(t+\delta t, \vec{x})-v_{\alpha}(t, \vec{x})\right) .
$$

Thus, the discretized kinetic energy theorem is

$$
\begin{aligned}
\partial_{t}\left(\frac{1}{2} v_{\alpha} v_{\alpha}\right)(t, \vec{x}) & =\left[\left(v_{\alpha} v_{\alpha}\right)(t+\delta t, \vec{x})-\left(v_{\alpha} v_{\alpha}\right)(t, \vec{x})\right] /(2 \delta \\
& =\frac{1}{2} f_{\alpha}(t, \vec{x})\left(v_{\alpha}(t+\delta t, \vec{x})+v_{\alpha}(t, \vec{x})\right)
\end{aligned}
$$

which indicates that half of the quantity to be averaged is not defined at coinciding but at successive times and the associated response function has a non-zero contribution. In conclusion, the average injected power is precisely defined as

$$
\begin{aligned}
\left\langle f_{\alpha}(t, \vec{x}) v_{\alpha}(t, \vec{x})\right\rangle= & \lim _{\delta t \rightarrow 0^{+}} \int_{\vec{x}^{\prime}} N_{\kappa, \alpha \beta}\left(\left|\vec{x}-\vec{x}^{\prime}\right|\right) \\
& \times\left\langle v_{\alpha}(t+\delta t, \vec{x}) \bar{v}_{\beta}\left(t, \vec{x}^{\prime}\right)\right\rangle,
\end{aligned}
$$

which, apart from the correct limit process and the one half factor, coincides with the expression (C2).

\section{APPENDIX D: NUMERICAL INTEGRATION OF THE LO FLOW EQUATIONS}

In this Appendix, we expound the detail of the numerical procedure implemented to integrate the LO flow equa- tions (71). The running functions $\hat{h}^{\nu, D}(\hat{\vec{p}})$ only depend on the modulus $\hat{p}$ of the wave-vector $\hat{\vec{p}}$. The dimensionless wave-numbers are discretized on a $\sqrt{\hat{p}}$ grid of typical size $\sqrt{\hat{p}_{\max }} \simeq 30$ and spacing $\Delta \equiv \Delta \sqrt{\hat{p}} \simeq 1 / 20$. The integrals over the internal wave-vector $\hat{\vec{q}}$ are calculated numerically using Simpson's rule, in cartesian coordinates, chosen with the $\hat{q}_{1}$ axis along the external wave-vector $\hat{\vec{p}}$ and the $(d-1)$ other axes $\hat{q}_{i}$ spanning the hyperplane perpendicular to $\hat{\vec{p}}$. With this choice, one simply has $\hat{\vec{p}} \cdot \hat{\vec{q}}=\hat{p} \hat{q}_{1}$ and $(\hat{\vec{p}}+\hat{\vec{q}})^{2}=\left(\hat{p}+\hat{q}_{1}\right)^{2}+\sum_{i=2}^{d} \hat{q}_{i}^{2}$. As a square root grid is used for the wave-numbers, the values of the functions $\hat{h}^{\nu, D}(\hat{\vec{p}}+\hat{\vec{q}})$ for arguments $|\hat{\vec{p}}+\hat{\vec{q}}|^{1 / 2}$ not falling onto mesh points are interpolated using cubic splines.

The presence of the $\partial_{s} \mathcal{R}_{\kappa}$ terms in (67) and (68) ensures that the integrands decrease exponentially with $\hat{q}$, such that the internal wave-number integral can be safely cut at an upper finite bound $\hat{p}_{\text {up }} \leq \hat{p}_{\max }$. For wavenumbers such that $|\hat{\vec{p}}+\hat{\vec{q}}|>\hat{p}_{\max }$, the functions $\hat{h}^{\nu, D}(\hat{\vec{p}}+\hat{\vec{q}})$ are extended outside the grid using power law extrapolations. This corresponds to the expected asymptotics of the flowing functions, at least close to the fixed point. The derivative terms $\hat{p} \partial_{\hat{p}}$ are computed using 5-point differences. For the propagation in renormalization time $s$, explicit Euler time stepping is used with a typical time step $\Delta s=-1 \times 10^{-4}$. Starting at $s=0$ from the bare action $\left(\hat{h}^{\nu}(\hat{\vec{p}})=1\right.$ and $\left.\hat{h}^{D}(\hat{\vec{p}})=0\right)$, we observe that the two functions $\hat{h}^{\nu, D}$ are smoothly deformed from their flat initial shapes to acquire their fixed point profiles, typically after $|s| \gtrsim 8$. The fixed point profiles are recorded at $s=-30$ (e.g. in Fig. 2).

\section{APPENDIX E: WARD IDENTITIES}

In this Appendix, we derive the Ward identities for the vertex functions which originate in the time-gauged shift and Galilean symmetries.

\section{Ward identities for the time-gauged shift symmetry}

Let us consider the (functional) Ward identity (26) associated with the time-gauged shift symmetry, and rewrite it using the explicit Ward identity for the pressure sector:

$$
\frac{\delta \Gamma_{\kappa}}{\delta \bar{p}(\mathbf{x})}=\frac{\delta \mathcal{S}_{0}}{\delta \bar{p}(\mathbf{x})}=\partial_{\alpha} u_{\alpha}
$$

One obtains

$$
\int_{\vec{x}}\left\{\frac{\delta \Gamma_{\kappa}}{\delta \bar{u}_{\beta}(\mathbf{x})}+u_{\beta}(\mathbf{x}) \partial_{\gamma} u_{\gamma}(\mathbf{x})\right\}=\int_{\vec{x}} \partial_{t} u_{\beta}(\mathbf{x}) .
$$

Differentiating this equation with respect to $\bar{u}_{\alpha}\left(t_{y}, \vec{y}\right)$ and evaluating it at vanishing fields, one obtains

$$
\int_{\vec{x}} \Gamma_{\beta \alpha}^{(0,2)}\left(t, \vec{x}, t_{y}, \vec{y}\right)=0
$$


or equivalently in Fourier space

$$
\Gamma_{\alpha \beta}^{(0,2)}(\omega, \vec{p}=\overrightarrow{0})=0
$$

Similarly, differentiating Eq. (E2) with respect to $u_{\alpha}\left(t_{y}, \vec{y}\right)$ and evaluating it at vanishing fields yields

$$
\int_{\vec{x}} \Gamma_{\alpha \beta}^{(1,1)}\left(t_{y}, \vec{y}, t, \vec{x}\right)=\int_{\vec{x}} \delta_{\alpha \beta} \partial_{t} \delta\left(t-t_{y}\right) \delta^{d}(\vec{x}-\vec{y})
$$

that is in Fourier space

$$
\Gamma_{\alpha \beta}^{(1,1)}(\omega, \vec{p}=\overrightarrow{0})=i \omega \delta_{\alpha \beta}
$$

Lastly, taking two derivatives with respect to $u_{\alpha}\left(t_{y}, \vec{y}\right)$ and $u_{\gamma}\left(t_{z}, \vec{z}\right)$ of Eq. (E2), one obtains the identity

$$
\int_{\vec{x}}\left\{\Gamma_{\alpha \gamma \beta}^{(2,1)}\left(t_{y}, \vec{y}, t_{z}, \vec{z}, t, \vec{x}\right)+\delta_{\alpha \beta} \partial_{\gamma}^{y} \delta\left(t_{y}-t_{z}\right) \delta^{d}(\vec{y}-\vec{z})+\delta_{\beta \gamma} \partial_{\alpha}^{z} \delta\left(t_{z}-t_{y}\right) \delta^{d}(\vec{z}-\vec{y})\right\}=0
$$

which yields in Fourier space, relabeling the indices

$$
\Gamma_{\alpha \beta \gamma}^{(2,1)}\left(\omega_{1}, \vec{p}_{1}, \omega_{2},-\vec{p}_{1}\right)=i p_{1}^{\alpha} \delta_{\beta \gamma}-i p_{1}^{\beta} \delta_{\alpha \gamma}
$$

By taking additional derivatives of (E3) and (E7) with respect to either fields $\vec{u}$ or $\overline{\vec{u}}$ and evaluating the resulting identity at zero external fields, one can infer the general property

$$
\Gamma_{\alpha_{1}, \cdots, \alpha_{n+m}}^{(m, n)}\left(\omega_{1}, \vec{p}_{1}, \cdots, \omega_{m}, \vec{p}_{m}, \omega_{m+1}, \vec{p}_{m+1}=\overrightarrow{0}, \cdots\right)=0 \quad \text { for all }(m, n) \text { but }(1,1) \text { and }(2,1)
$$

which means that any $(m, n)$-point vertex function with a zero wave-vector on a $\overline{\vec{u}}$-leg vanishes, except the functions $(m, n)=(1,1)$ and $(m, n)=(2,1)$ which keep their bare forms (E6) and (E8).

\section{Ward identities for the time-gauged Galilean symmetry}

Let us derive the (functional) Ward identities ensuing from the time-gauged Galilean symmetry. Retaining only the terms which give a non-zero contribution at vanishing fields in the velocity sector (i.e. dropping the pressure terms), the Ward identity (24) reads

$$
\int_{\vec{x}}\left\{\partial_{\alpha} u_{\beta}(t, \vec{x}) \frac{\delta \Gamma_{\kappa}}{\delta u_{\beta}(t, \vec{x})}+\partial_{t} \frac{\delta \Gamma_{\kappa}}{\delta u_{\alpha}(t, \vec{x})}+\partial_{\alpha} \bar{u}_{\beta}(t, \vec{x}) \frac{\delta \Gamma_{\kappa}}{\delta \bar{u}_{\beta}(t, \vec{x})}\right\}=-\int_{\vec{x}} \partial_{t}^{2} \bar{u}_{\alpha}(t, \vec{x}) .
$$

Differentiating this equation with respect to $\overline{\vec{u}}_{\beta}\left(t_{y}, \vec{y}\right)$, and evaluating the resulting identity at vanishing fields, one obtains

$$
\delta_{\alpha \beta} \partial_{t}^{2} \delta\left(t-t^{\prime}\right)+\int_{\vec{x}} \partial_{t} \Gamma_{\alpha \beta}^{(1,1)}\left(t, \vec{x}, t_{y}, \vec{y}\right)=0
$$

which leads in Fourier space to

$$
\Gamma_{\alpha \beta}^{(1,1)}(\omega, \vec{p}=\overrightarrow{0})=i \omega \delta_{\alpha \beta}
$$

Then, taking two derivatives of Eq. (E10) with respect to $u_{\mu}\left(t_{y}, \vec{y}\right)$ and $\bar{u}_{\nu}\left(t_{z}, \vec{z}\right)$ yields at vanishing fields

$$
\int_{\vec{x}}\left\{\partial_{t_{x}} \Gamma_{\alpha \mu \nu}^{(2,1)}\left(t_{x}, \vec{x}, t_{y}, \vec{y}, t_{z}, \vec{z}\right)-\delta\left(t_{x}-t_{y}\right) \delta(\vec{x}-\vec{y}) \partial_{\alpha} \Gamma_{\mu \nu}^{(1,1)}\left(t_{y}, \vec{y}, t_{z}, \vec{z}\right)-\delta\left(t_{x}-t_{z}\right) \delta(\vec{x}-\vec{z}) \partial_{\alpha} \Gamma_{\mu \nu}^{(1,1)}\left(t_{y}, \vec{y}, t_{z}, \vec{z}\right)\right\}=0
$$

This provides in Fourier space an exact identity relating the 3 -point vertex $\Gamma^{(2,1)}$ with a zero wave-vector on a $\vec{u}$-leg to the 2-point function $\Gamma^{(1,1)}$, which reads

$$
\Gamma_{\alpha \beta \gamma}^{(2,1)}\left(\omega_{1}, \vec{p}_{1}=\overrightarrow{0} ; \omega_{2}, \vec{p}_{2}\right)=-\frac{p_{2}^{\alpha}}{\omega_{1}}\left(\Gamma_{\beta \gamma}^{(1,1)}\left(\omega_{1}+\omega_{2}, \vec{p}_{2}\right)-\Gamma_{\beta \gamma}^{(1,1)}\left(\omega_{2}, \vec{p}_{2}\right)\right) .
$$


Similarly, taking two derivatives of Eq. E10 with respect to $\bar{u}$, one obtains at vanishing fields

$$
\int_{\vec{x}}\left\{\partial_{t_{x}} \Gamma_{\alpha \mu \nu}^{(1,2)}\left(t_{x}, \vec{x}, t_{y}, \vec{y}, t_{z}, \vec{z}\right)-\delta\left(t_{x}-t_{y}\right) \delta(\vec{x}-\vec{y}) \partial_{\alpha} \Gamma_{\mu \nu}^{(0,2)}\left(t_{y}, \vec{y}, t_{z}, \vec{z}\right)-\delta\left(t_{x}-t_{z}\right) \delta(\vec{x}-\vec{z}) \partial_{\alpha} \Gamma_{\mu \nu}^{(0,2)}\left(t_{y}, \vec{y}, t_{z}, \vec{z}\right)\right\}=0
$$

This yields in Fourier space an exact identity relating the 3 -point vertex $\Gamma^{(1,2)}$ with a zero wave-vector on its $\vec{u}$-leg to the 2-point function $\Gamma^{(0,2)}$ as

$$
\Gamma_{\alpha \beta \gamma}^{(1,2)}\left(\omega_{1}, \vec{p}_{1}=\overrightarrow{0} ; \omega_{2}, \vec{p}_{2}\right)=-\frac{p_{2}^{\alpha}}{\omega_{1}}\left(\Gamma_{\beta \gamma}^{(0,2)}\left(\omega_{1}+\omega_{2}, \vec{p}_{2}\right)-\Gamma_{\beta \gamma}^{(0,2)}\left(\omega_{2}, \vec{p}_{2}\right)\right)
$$

As for the 4-point vertices, taking one additional derivative of Eq. (E13) with respect to $\bar{u}_{\rho}\left(t_{s}, \vec{s}\right)$ and evaluating the obtained identity at vanishing fields yields

$$
\begin{aligned}
& \int_{\vec{x}}\left\{\partial_{t_{x}} \Gamma_{\alpha \mu \rho \nu}^{(2,2)}\left(t_{x}, \vec{x}, t_{y}, \vec{y}, t_{s}, \vec{s}, t_{z}, \vec{z}\right)-\delta\left(t_{x}-t_{y}\right) \delta(\vec{x}-\vec{y}) \partial_{\alpha} \Gamma_{\mu \rho \nu}^{(1,2)}\left(t_{y}, \vec{y}, t_{s}, \vec{s}, t_{z}, \vec{z}\right)\right. \\
& \left.-\delta\left(t_{x}-t_{z}\right) \delta(\vec{x}-\vec{z}) \partial_{\alpha} \Gamma_{\mu \rho \nu}^{(1,2)}\left(t_{y}, \vec{y}, t_{s}, \vec{s}, t_{z}, \vec{z}\right)-\delta\left(t_{x}-t_{s}\right) \delta(\vec{x}-\vec{s}) \partial_{\alpha} \Gamma_{\mu \rho \nu}^{(1,2)}\left(t_{y}, \vec{y}, t_{s}, \vec{s}, t_{z}, \vec{z}\right)\right\}=0
\end{aligned}
$$

Similarly, taking one additional derivative of Eq. (E13) with respect to $u_{\rho}\left(t_{s}, \vec{s}\right)$ and evaluating the ensuing identity at zero fields leads to

$$
\begin{aligned}
& \int_{\vec{x}}\left\{\partial_{t_{x}} \Gamma_{\alpha \mu \rho \nu}^{(3,1)}\left(t_{x}, \vec{x}, t_{y}, \vec{y}, t_{s}, \vec{s}, t_{z}, \vec{z}\right)-\delta\left(t_{x}-t_{y}\right) \delta(\vec{x}-\vec{y}) \partial_{\alpha} \Gamma_{\mu \rho \nu}^{(2,1)}\left(t_{y}, \vec{y}, t_{s}, \vec{s}, t_{z}, \vec{z}\right)\right. \\
& \left.-\delta\left(t_{x}-t_{z}\right) \delta(\vec{x}-\vec{z}) \partial_{\alpha} \Gamma_{\mu \rho \nu}^{(2,1)}\left(t_{y}, \vec{y}, t_{s}, \vec{s}, t_{z}, \vec{z}\right)-\delta\left(t_{x}-t_{s}\right) \delta(\vec{x}-\vec{s}) \partial_{\alpha} \Gamma_{\mu \rho \nu}^{(2,1)}\left(t_{y}, \vec{y}, t_{s}, \vec{s}, t_{z}, \vec{z}\right)\right\}=0
\end{aligned}
$$

Fourier transforming the two previous relations, one deduces two exact identities relating the 4 -point vertices $\Gamma^{(2,2)}$ and $\Gamma^{(3,1)}$ with one zero wave-vector on a $\vec{u}$-leg to 3 -point functions

$$
\begin{aligned}
\Gamma_{\alpha \beta \gamma \delta}^{(2,2)}\left(\omega_{1}, \vec{p}_{1}=\overrightarrow{0}, \omega_{2}, \vec{p}_{2}, \omega_{3}, \vec{p}_{3}\right) & =-\frac{1}{\omega_{1}}\left[p_{2}^{\alpha} \Gamma_{\beta \gamma \delta}^{(1,2)}\left(\omega_{1}+\omega_{2}, \vec{p}_{2}, \omega_{3}, \vec{p}_{3}\right)+p_{3}^{\alpha} \Gamma_{\beta \gamma \delta}^{(1,2)}\left(\omega_{2}, \vec{p}_{2}, \omega_{1}+\omega_{3}, \vec{p}_{3}\right)\right. \\
& \left.+\left(-p_{2}-p_{3}\right)^{\alpha} \Gamma_{\beta \gamma \delta}^{(1,2)}\left(\omega_{2}, \vec{p}_{2}, \omega_{3}, \vec{p}_{3}\right)\right] \\
\Gamma_{\alpha \beta \gamma \delta}^{(3,1)}\left(\omega_{1}, \vec{p}_{1}=\overrightarrow{0}, \omega_{2}, \vec{p}_{2}, \omega_{3}, \vec{p}_{3}\right) & =-\frac{1}{\omega_{1}}\left[p_{2}^{\alpha} \Gamma_{\beta \gamma \delta}^{(2,1)}\left(\omega_{1}+\omega_{2}, \vec{p}_{2}, \omega_{3}, \vec{p}_{3}\right)+p_{3}^{\alpha} \Gamma_{\beta \gamma \delta}^{(2,1)}\left(\omega_{2}, \vec{p}_{2}, \omega_{1}+\omega_{3}, \vec{p}_{3}\right)\right. \\
& \left.+\left(-p_{2}-p_{3}\right)^{\alpha} \Gamma_{\beta \gamma \delta}^{(2,1)}\left(\omega_{2}, \vec{p}_{2}, \omega_{3}, \vec{p}_{3}\right)\right] .
\end{aligned}
$$

\section{APPENDIX F: EXACT FLOW EQUATIONS FOR THE 2-POINT VERTEX FUNCTIONS IN THE LARGE EXTERNAL WAVE-NUMBER LIMIT}

In this Appendix, we derive an expression for the flow equations of $\Gamma_{\perp}^{(0,2)}(\nu, \vec{p})$ and $\Gamma_{\perp}^{(1,1)}(\nu, \vec{p})$ which becomes exact in the limit of large external wave-number $|\vec{p}| \gg \kappa$. The diagrams entering these flow equations are schematically depicted in Figs. 4 and 5. Some of them, diagrams (a), (b), (d) of Fig. 4 and diagrams (a), (b), (f) of Fig. 5 are vanishing (see Sec. VII). We calculate below the contributions of the remaining non-zero diagrams.

\section{Flow equation of $\Gamma_{\perp}^{(0,2)}(\nu, \vec{p})$ in the large $\vec{p}$ limit}

We separately analyze the three diagrams (c), (d) and (e) of Fig. 5 which give non-vanishing contributions to the flow of $\Gamma_{\perp}^{(0,2)}$. We begin with determining the expression of diagram (d) in the limit of large external wave-number 
$|\vec{p}| \gg \kappa$. Introducing the operator $\tilde{\partial}_{s} \equiv \partial_{s} R_{\kappa} \frac{\partial}{\partial R_{\kappa}}+\partial_{s} N_{\kappa} \frac{\partial}{\partial N_{\kappa}}$, this contribution may be written as

$$
\left[\partial_{s} \Gamma_{\alpha \beta}^{(0,2)}(\nu, \vec{p})\right]_{(d)}=-\frac{1}{2} \tilde{\partial}_{s} \int_{\omega, \vec{q}} \Gamma_{i j \alpha}^{(2,1)}(\omega, \vec{q},-\omega-\nu,-\vec{p}-\vec{q}) G_{j k}^{u u}(\omega+\nu, \vec{p}+\vec{q}) \Gamma_{k l \beta}^{(2,1)}(\omega+\nu, \vec{p}+\vec{q},-\omega,-\vec{q}) G_{l i}^{u u}(\omega, \vec{q}) .
$$

Either the operator $\tilde{\partial}_{s}$ acts on $G_{l i}^{u u}(\omega, \vec{q})$ and the internal wave-vector $\vec{q}$ is cut off to $|\vec{q}| \lesssim \kappa$ so that it is negligible compared to $\vec{p}$ and can be set to zero. Or it acts on $G_{j k}^{u u}(\omega+\nu, \vec{p}+\vec{q})$, in which case the combination $\vec{p}+\vec{q}$ is cut off. Changing variables, this last contribution identifies with the first one. Hence, in the large $|\vec{p}|$ limit, the flow equation (F1) becomes

$$
\left[\partial_{s} \Gamma_{\alpha \beta}^{(0,2)}(\nu, \vec{p})\right]_{(d)}=-\int_{\omega} \Gamma_{i j \alpha}^{(2,1)}(\omega, \overrightarrow{0},-\omega-\nu,-\vec{p}) G_{j k}^{u u}(\omega+\nu, \vec{p}) \Gamma_{k l \beta}^{(2,1)}(\omega+\nu, \vec{p},-\omega, \overrightarrow{0}) \tilde{\partial}_{s} \int_{\vec{q}} G_{l i}^{u u}(\omega, \vec{q})
$$

Then, using the Ward identity (85) and projecting onto the transverse sector, one deduces

$$
\begin{aligned}
P_{\alpha \beta}^{\perp}(\vec{p})\left[\partial_{s} \Gamma_{\alpha \beta}^{(0,2)}(\nu, \vec{p})\right]_{(d)} & =-(d-1)\left(1-\frac{1}{d}\right) p^{2} \int_{\omega} \frac{1}{\omega^{2}}\left(\Gamma_{\perp}^{(1,1)}(-\nu, \vec{p})-\Gamma_{\perp}^{(1,1)}(-\nu-\omega, \vec{p})\right) \\
& \times\left(\Gamma_{\perp}^{(1,1)}(\nu, \vec{p})-\Gamma_{\perp}^{(1,1)}(\nu+\omega, \vec{p})\right) G_{\perp}^{(2,0)}(\omega+\nu, \vec{p}) \tilde{\partial}_{s} \int_{\vec{q}} G_{\perp}^{(2,0)}(\omega, \vec{q})
\end{aligned}
$$

where parity in $\vec{p}$ and the identity $\int_{\vec{q}}(\vec{p} \cdot \vec{q})^{2} f\left(q^{2}\right)=\frac{p^{2}}{d} \int_{\vec{q}} q^{2} f\left(q^{2}\right)$ were used. The contribution of diagram (c) of Fig. 5 can be written as

$$
\begin{aligned}
{\left[\partial_{s} \Gamma_{\alpha \beta}^{(0,2)}(\nu, \vec{p})\right]_{(c)} } & =-\int_{\omega, \vec{q}} \Gamma_{i \alpha j}^{(1,2)}(\omega, \vec{q}, \nu, \vec{p}) G_{j k}^{u \bar{u}}(-\omega-\nu, \vec{p}+\vec{q}) \Gamma_{k l \beta}^{(2,1)}(\omega+\nu, \vec{p}+\vec{q},-\omega,-\vec{q}) \tilde{\partial}_{s} G_{l i}^{u u}(\omega, \vec{q})+c . c . \\
& =-\int_{\vec{q}} \Gamma_{i \alpha j}^{(1,2)}(\omega, \overrightarrow{0}, \nu, \vec{p}) G_{j k}^{u \bar{u}}(-\omega-\nu, \vec{p}) \Gamma_{k l \beta}^{(2,1)}(\omega+\nu, \vec{p},-\omega, \overrightarrow{0}) \tilde{\partial}_{s} \int_{\vec{q}} G_{l i}^{u u}(\omega, \vec{q})+c . c .
\end{aligned}
$$

where the second equality holds in the large $|\vec{p}|$ limit, when the internal wave-vector $\vec{q}$ is negligible compared to $\vec{p}$. Inserting the Ward identities (85) and (86) for the 3-point vertices and projecting onto the transverse sector, one obtains

$$
\begin{aligned}
P_{\alpha \beta}^{\perp}(\vec{p})\left[\partial_{s} \Gamma_{\alpha \beta}^{(0,2)}(\nu, \vec{p})\right]_{(c)} & =-\frac{(d-1)^{2}}{d} p^{2} \int_{\omega}\left[\frac{\Gamma_{\perp}^{(0,2)}(\omega+\nu, \vec{p})-\Gamma_{\perp}^{(0,2)}(\nu, \vec{p})}{\omega}\right] \times\left[\frac{\Gamma_{\perp}^{(1,1)}(\omega+\nu, \vec{p})-\Gamma_{\perp}^{(1,1)}(\nu, \vec{p})}{\omega}\right] \\
& \times G_{\perp}^{u \bar{u}}(-\omega-\nu, \vec{p}) \tilde{\partial}_{s} \int_{\vec{q}} G_{\perp}^{u u}(\omega, \vec{q})+c . c . .
\end{aligned}
$$

Similarly, the contribution of diagram (e) of Fig. 5 simplifies to

$$
\left[\partial_{s} \Gamma_{\alpha \beta}^{(0,2)}(\nu, \vec{p})\right]_{(e)}=\frac{1}{2} \tilde{\partial}_{s} \int_{\omega, \vec{q}} \Gamma_{i j \alpha \beta}^{(2,2)}(\omega, \vec{q},-\omega,-\vec{q}, \nu, \vec{p}) G_{i j}^{u u}(\omega, \vec{q})=\frac{1}{2} \tilde{\partial}_{s} \int_{\omega} \Gamma_{i j \alpha \beta}^{(2,2)}(\omega, \overrightarrow{0},-\omega, \overrightarrow{0}, \nu, \vec{p}) \int_{\vec{q}} G_{i j}^{u u}(\omega, \vec{q})
$$

which transverse projection reads, using the Ward identity (87) for the 4-point vertex function with two vanishing wave-vectors on its $\vec{u}$-legs,

$$
P_{\alpha \beta}^{\perp}(\vec{p})\left[\partial_{s} \Gamma_{\alpha \beta}^{(0,2)}(\nu, \vec{p})\right]_{(e)}=\frac{1}{2} \frac{(d-1)^{2}}{d} p^{2} \int_{\omega} \frac{1}{\omega^{2}}\left[\Gamma_{\perp}^{(0,2)}(\omega+\nu, \vec{p})-2 \Gamma_{\perp}^{(0,2)}(\nu, \vec{p})+\Gamma_{\perp}^{(0,2)}(-\omega+\nu, \vec{p})\right] \tilde{\partial}_{s} \int_{\vec{q}} G_{\perp}^{u u}(\omega, \vec{q})
$$

The exact flow equation of $\Gamma_{\perp}^{(0,2)}(\nu, \vec{p})$ in the large external wave-number limit is the sum of the three contributions (F3), (F5) and (F7), which yields Eq. (90). 


\section{Flow equation of $\Gamma_{\perp}^{(1,1)}(\nu, \vec{p})$ in the large $\vec{p}$ limit}

Only the two diagrams (c) and (e) of Fig. 4 give a non-vanishing contribution to the flow of $\Gamma_{\perp}^{(1,1)}$ in the large external wave-number limit. The contribution of diagram (c) can be written as

$$
\begin{aligned}
{\left[\partial_{s} \Gamma_{\alpha \beta}^{(1,1)}(\nu, \vec{p})\right]_{(c)}=} & -\tilde{\partial}_{s} \int_{\omega, \vec{q}} \Gamma_{i \alpha j}^{(2,1)}(\omega, \vec{q}, \nu, \vec{p}) G_{j k}^{u \bar{u}}(-\omega-\nu, \vec{p}+\vec{q}) \Gamma_{k l \beta}^{(2,1)}(\omega+\nu, \vec{p}+\vec{q},-\omega,-\vec{q}) G_{l i}^{u u}(\omega, \vec{q}) \\
= & -\int_{\omega, \vec{q}} \Gamma_{i \alpha j}^{(2,1)}(\omega, \overrightarrow{0}, \nu, \vec{p}) G_{j k}^{u \bar{u}}(-\omega-\nu, \vec{p}) \Gamma_{k l \beta}^{(2,1)}(\omega+\nu, \vec{p},-\omega, \overrightarrow{0}) \tilde{\partial}_{s} G_{l i}^{u u}(\omega, \vec{q}) \\
& -\int_{\omega, \vec{q}} \Gamma_{i \alpha j}^{(2,1)}(-\omega-\nu,-\vec{p}, \nu, \vec{p}) G_{l i}^{u u}(-\nu-\omega, \vec{q}+\vec{p}) \Gamma_{k l \beta}^{(2,1)}(-\omega,-\vec{q}, \omega+\nu, \vec{p}) \tilde{\partial}_{s} G_{j k}^{u \bar{u}}(\omega, \vec{q})
\end{aligned}
$$

where again, the second equality holds in the large $\vec{p}$ limit, when the internal wave-vector $\vec{q}$ can be set to zero. The transverse projection of this expression, inserting the Ward identity (85), is given by

$$
P_{\alpha \beta}^{\perp}(\vec{p})\left[\partial_{s} \Gamma_{\alpha \beta}^{(1,1)}(\nu, \vec{p})\right]_{(c)}=-\frac{(d-1)^{2}}{d} p^{2} \int_{\omega}\left[\frac{\Gamma_{\perp}^{(1,1)}(\omega+\nu, \vec{p})-\Gamma_{\perp}^{(1,1)}(\nu, \vec{p})}{\omega}\right]^{2} G_{\perp}^{u \bar{u}}(-\omega-\nu, \vec{p}) \tilde{\partial}_{s} \int_{\vec{q}} G_{\perp}^{u u}(\omega, \vec{q}) .
$$

Lastly, the contribution of diagram (e) of Fig. 4 is very similar to the one of diagram (e) in the flow of $\Gamma_{\perp}^{(0,2)}$ and its transverse projection, using the Ward identity (88), is given by

$$
\begin{aligned}
P_{\alpha \beta}^{\perp}(\vec{p})\left[\partial_{s} \Gamma_{\alpha \beta}^{(1,1)}(\nu, \vec{p})\right]_{(e)} & =\frac{1}{2} P_{\alpha \beta}^{\perp}(\vec{p}) \tilde{\partial}_{s} \int_{\omega, \vec{q}} \Gamma_{i j \alpha \beta}^{(3,1)}(\omega, \overrightarrow{0},-\omega, \overrightarrow{0}, \nu, \vec{p}) G_{i j}^{u u}(\omega, \vec{q}) \\
& =\frac{1}{2} P_{\alpha \beta}^{\perp}(\vec{p}) \int_{\omega} \Gamma_{i j \alpha \beta}^{(3,1)}(\omega, \overrightarrow{0},-\omega, \overrightarrow{0}, \nu, \vec{p}) \tilde{\partial}_{s} \int_{\vec{q}} G_{i j}^{u u}(\omega, \vec{q}) \\
& =\frac{1}{2} \frac{(d-1)^{2}}{d} p^{2} \int_{\omega} \frac{1}{\omega^{2}}\left[\Gamma_{\perp}^{(1,1)}(\omega+\nu, \vec{p})-2 \Gamma_{\perp}^{(1,1)}(\nu, \vec{p})+\Gamma_{\perp}^{(1,1)}(-\omega+\nu, \vec{p})\right] \tilde{\partial}_{s} \int_{\vec{q}} G_{\perp}^{u u}(\omega, \vec{q}) .
\end{aligned}
$$

The exact flow equation of $\Gamma_{\perp}^{(1,1)}(\nu, \vec{p})$ in the large external wave-number limit is the sum of the two contributions (F9) and (F10), which yields Eq. (89).

[1] A. Kolmogorov, Dokl. Akad. Nauk SSSR 30, 299 (1941).

[2] A. Kolmogorov, Dokl. Akad. Nauk SSSR 31, 538 (1941).

[3] A. Kolmogorov, Dokl. Akad. Nauk SSSR 32, 16 (1941).

[4] U. Frisch, Turbulence: the legacy of A.N. Kolmogorov (Cambridge University Press, ADDRESS, 1995).

[5] A. Oboukhov, J. Fluid Mech. 13, 77 (1962).

[6] R. Kraichnan, Phys. Fluids 10, 1417 (1967).

[7] G. K. Batchelor, Phys. Fluids Suppl. II 12, 233 (1969).

[8] M. Lesieur, Turbulence in Fluids, 2nd edition ed. (Kluwer, Dordrecht, 1990).

[9] G. Eyink and N. Goldenfeld, Phys. Rev. E 50, 4679 (1994).

[10] J. Zinn-Justin, Quantum Field Theory and Critical Phenomena, 3rd ed. (Oxford University Press, New York, 1989).

[11] L. Smith and S. Woodruff, Annu. Rev. Fluid Mech. 30, 275 (1998).

[12] L. Adzhemyan, N. Antonov, and A. N. Vasil'ev, The Field Theoretic Renormalization Group in Fully Devel- oped Turbulence (Gordon and Breach, London, 1999).

[13] Y. Zhou, Phys. Rept. 488, 1 (2010).

[14] C. de Dominicis and P. Martin, Phys. Rev. A 19, 419 (1979).

[15] J. Fournier and U. Frisch, Phys. Rev. A 28, 1000 (1983).

[16] V. Yakhot and S. Orszag, J. Sci. Comput. 1, 3 (1986).

[17] V. Canuto and M. Dubovikov, Phys. Fluids 8, 571 (1996).

[18] L. Adzhemyan, N. Antonov, M. Kompaniets, and A. Vasil'ev, Int. J. Mod. Phys. 17, 2137 (2003).

[19] L. Adzhemyan et al., J. Phys. A 41, 495002 (2008).

[20] P. Olla, Phys. Rev. Lett. 67, 2465 (1991).

[21] J. Honkonen and M. Y. Nalimov, Z. Phys. B 99, 297 (1996).

[22] N. V. Antonov and A. V. Runov, Theor. Math. Phys. 112, 1131 (1997).

[23] J. Honkonen, Phys. Rev. E 58, 4532 (1998).

[24] J. Honkonen, Y. S. Kabrits, and M. V. Kompaniets, arXiv:nlin/0201025 (2002). 
[25] L. T. Adzhemyan, J. Honkonen, M. V. Kompaniets, and A. N. Vasil'ev, Phys. Rev. E 71, 036305 (2005).

[26] J. Mayo, Phys. Rev. E 72, 056316 (2005).

[27] R. H. Kraichnan, Phys. Rev. Lett. 72, 1016 (1994).

[28] K. Gawedzki and A. Kupianen, Phys. Rev. Lett. 75, 3834 (1995).

[29] M. Chertkov, G. Falkovich, I. Kolokolov, and V. Lebedev, Phys. Rev. E 52, 4924 (1995).

[30] B. I. Shraiman and E. D. Siggia, C. R. Acad. Sci., Ser. I: Math. 321, 279 (1995).

[31] M. Chertkov and G. Falkovich, Phys. Rev. Lett. 76, 2706 (1996).

[32] D. Bernard, K. Gawe, dzki, and A. Kupiainen, Phys. Rev. E 54, 2564 (1996).

[33] A. L. Fairhall, O. Gat, V. L'vov, and I. Procaccia, Phys. Rev. E 53, 3518 (1996).

[34] O. Gat, V. S. L'vov, E. Podivilov, and I. Procaccia, Phys. Rev. E 55, R3836 (1997).

[35] U. Frisch, A. Mazzino, and M. Vergassola, Phys. Rev. Lett. 80, 5532 (1998).

[36] L. Adzhemyan, N. Antonov, and A. Vasilev, Phys. Rev. E 58, 1823 (1998).

[37] A. Mazzino and P. Muratore-Ginanneschi, Phys. Rev. E 63, 015302(R) (2001).

[38] G. Falkovich, K. Gawedzki, and M. Vergassola, Rev. Mod. Phys. 73, 913 (2001).

[39] N. V. Antonov, J. Phys. A 39, 7825 (2006).

[40] L. Adzhemyan, N. Antonov, and A. Vasilev, Theor. Math. Phys. 120, 1074 (1999).

[41] L. Adzhemyan, N. Antonov, V. Barinov, and Y. K. A. Vasil'ev, Phys. Rev. E 63, 025303 (2001).

[42] L. Adzhemyan, N. Antonov, V. Barinov, and Y. K. A. Vasil'ev, Phys. Rev. E 64, 019901 (2001).

[43] M. Giles, J. Phys. A 34, 4389 (2001).

[44] V. Belinicher and V. L'vov, Sov. Phys. JETP 66, 303 (1987).

[45] V. L'vov and I. Procaccia, in Exact resummations in the theory of hydrodynamic turbulence: 0. Line-resummed diagrammatic perturbation approach (in: F. David, P. Ginsparg, and J. Zinn-Justin (Eds.) Les Houches session LXII, 1994, "Fluctuating Geometries in Statistical Mechanics and Field Theory", Elsevier, Amsterdam, 1995).

[46] V. L'vov and I. Procaccia, Phys. Rev. Lett. 74, 4684 (1994).

[47] V. L'vov and I. Procaccia, Phys. Rev. E 52, 3840 (1995).

[48] V. L'vov and I. Procaccia, Phys. Rev. E 52, 3858 (1995).

[49] V. L'vov and I. Procaccia, Phys. Rev. E 53, 3468 (1996).

[50] V. L'vov and I. Procaccia, Phys. Rev. Lett. 76, 2898 (1996).

[51] V. L'vov and I. Procaccia, Phys. Rev. Lett. 77, 3541 (1996).

[52] V. L'vov and I. Procaccia, Phys. Rev. E 54, 6268 (1996).

[53] V. L'vov and I. Procaccia, Physica A 257, 165 (1998).

[54] V. Belinicher, V. L'vov, and I. Procaccia, Physica A 254, 215 (1998).

[55] V. Belinicher, V. L'vov, A. Pomyalov, and I. Procaccia, J. Stat. Phys. 93, 797 (1998).

[56] V. L'vov and I. Procaccia, Phys. Rev. E 62, 8037 (2000).

[57] C. Wetterich, Phys. Lett. B 301, 90 (1993).

[58] U. Ellwanger, Z. Phys. C 58, 619 (1993).

[59] U. Ellwanger, Z. Phys. C 62, 503 (1993).

[60] N. Tetradis and C. Wetterich, Nucl. Phys. B [FS] 422 , 541 (1994).
[61] T. R. Morris, Int. J. Mod. Phys. A 9, 2411 (1994).

[62] J. Berges, N. Tetradis, and C. Wetterich, Phys. Rep. 363, 223 (2002).

[63] M. Tissier, B. Delamotte, and D. Mouhanna, Phys. Rev. Lett. 84, 5208 (2000).

[64] M. Tissier, D. Mouhanna, and B. Delamotte, Phys. Rev. B 61, 15327 (2000).

[65] L. Canet, B. Delamotte, D. Mouhanna, and J. Vidal, Phys. Rev. D 67, 065004 (2003).

[66] L. Canet, B. Delamotte, D. Mouhanna, and J. Vidal, Phys. Rev. B 68, 064421 (2003).

[67] F. Benitez et al., Phys. Rev. E 80, 030103 (2009).

[68] K. Essafi, J.-P. Kownacki, and D. Mouhanna, Phys. Rev. Lett. 106, 128102 (2011).

[69] F. Benitez et al., Phys. Rev. E 85, 026707 (2012).

[70] G. Tarjus and M. Tissier, Phys. Rev. Lett. 93, 267008 (2004).

[71] L. Canet, H. Chaté, and B. Delamotte, Phys. Rev. Lett. 92, 255703 (2004).

[72] L. Canet et al., Phys. Rev. Lett. 95, 100601 (2005).

[73] M. Tissier and G. Tarjus, Phys. Rev. Lett. 96, 087202 (2006).

[74] M. Tissier and G. Tarjus, Phys. Rev.B 78, 024204 (2008).

[75] D. Gredat, H. Chaté, B. Delamotte, and I. Dornic, Phys. Rev. E 89, 010102 (2014).

[76] M. Kardar, G. Parisi, and Y.-C. Zhang, Phys. Rev. Lett. 56, 889 (1986).

[77] T. Halpin-Healy and Y. Zhang, Phys. Rep. 254, 215 (1995).

[78] K. J. Wiese, J. Stat. Phys. 93, 143 (1998).

[79] L. Canet, H. Chaté, B. Delamotte, and N. Wschebor, Phys. Rev. Lett. 104, 150601 (2010).

[80] L. Canet, H. Chaté, B. Delamotte, and N. Wschebor, Phys. Rev. E 84, 061128 (2011).

[81] T. Kloss, L. Canet, and N. Wschebor, Phys. Rev. E 86, 051124 (2012).

[82] T. Kloss, L. Canet, B. Delamotte, and N. Wschebor, Phys. Rev. E 89, 022108 (2014).

[83] T. Kloss, L. Canet, and N. Wschebor, Phys. Rev. E 90, 062133 (2014).

[84] P. Tomassini, Phys. Lett. B 411, 117 (1997).

[85] A. A. Fedorenko, P. Le Doussal, and K. J. Wiese, J. Stat. Mech. 2013, P04014 (2013).

[86] C. Mejía-Monasterio and P. Muratore-Ginanneschi, Phys. Rev. E 86, 016315 (2012).

[87] S. Mathey, T. Gasenzer, and J. M. Pawlowski, Phys. Rev. A 92, 023635 (2015).

[88] C. Pagani, arXiv:1505.01293 (2015).

[89] L. Canet, B. Delamotte, O. Deloubrière, and N. Wschebor, Phys. Rev. Lett. 92, 195703 (2004).

[90] L. Canet, H. Chaté, and B. Delamotte, J. Phys. A: Math. Theor. 44, 495001 (2011).

[91] E. V. Teodorovich, Appl. Math. and Mech. 53, 340 (1989).

[92] L. Adzhemyan, N. Antonov, and T. L. Kim, Theor. Math. Phys. 100, 1086 (1994).

[93] N. V. Antonov, S. V. Borisenok, and V. Girina, Theor. Math. Phys. 106, 75 (1996).

[94] A. Berera and D. Hochberg, Phys. Rev. Lett. 99, 254501 (2007).

[95] L. Canet, B. Delamotte, and N. Wschebor, Phys. Rev. E 91, 053004 (2015). 
[96] P. C. Martin, E. D. Siggia, and H. A. Rose, Phys. Rev. A 8, 423 (1973).

[97] H. K. Janssen, Z. Phys. B 23, 377 (1976).

[98] C. de Dominicis, J. Phys. Colloques 37, 247 (1976).

[99] Note that no Grassmann fields have been introduced, because the Itō's discretization has been chosen, in which the Jacobian involved in the MSRJD procedure is simply a constant (independent of the fields), absorbed in the normalization, see Ref. 95] for the detailed derivation of the generating functional. The consequences of this choice and of causality are expounded in Refs. [69, 90], and taken into account throughout this work.

[100] B. Delamotte and L. Canet, Condensed Matter Phys. 8, 163 (2005).

[101] F. Benitez and N. Wschebor, Phys. Rev. E 86, 010104 (2012).

[102] Whereas physical quantities do not depend on the shape of $R_{\kappa}$ in the exact theory, they acquire a (spurious) dependence on $a$ when approximations are performed. This parameter can therefore be utilized to optimize results [65, 66].

[103] C. Wetterich, Z. Phys. C 57, 451 (1993).

[104] A. Berera and D. Hochberg, Nucl. Phys. B 814, 522 (2009).

[105] T. von Kármán and L. Howarth, Proc. R. Soc. Lond. A 164, 192 (1938).

[106] G. Falkovich, I. Fouxon, and Y. Oz, J. Fluid Mech. 644, 465 (2010).

[107] This choice is justified because we only study the $\kappa \rightarrow 0$ limit where the dimensionless flow approaches a universal fixed point, where the precise energy dissipation mechanism at large $\kappa$ is washed out.
[108] K. Gawedzki, arXiv:chao-dyn/9907024 (1999).

[109] Note that in this limit, the mean rate of energy dissipation at the microscopic scale Eq. (40) is only logarithmically divergent, which corroborates the fact that energy is not dominantly dissipated at this scale in $d=2$.

[110] M. Prähofer and H. Spohn, J. Stat. Phys. 115, 255 (2004).

[111] T. Halpin-Healy, Phys. Rev. E 88, 042118 (2013).

[112] L. Canet, arXiv:cond-mat/0509541.

[113] Notice that the dependence of these values on the regulator (via the a parameter in Eq. (12) could also be studied. However, it is meaningless within the LO approximation since this approximation is not appropriate to study with precision the large wave-number sector, as already pointed out in Sec. VA

[114] As a remark, let us notice that the exact closure of the flow equations in the large wave-number sector constitutes an essential property of the BMW scheme. However, it generally requires to keep arbitrary external fields since within this scheme, the 3 - and 4- point vertex functions are expressed as derivatives of 2-point functions with respect to the fields. Here, the closure entirely relies on the symmetries, and thus can be achieved even at zero external fields. In that sense, the BMW scheme appears simpler here than in standard situations (e.g. in equilibrium scalar theories). The resulting approximation is very similar to the full SO approximation for the KPZ problem briefly presented in Sec. VA but with the difference that whereas the SO approximation for KPZ requires a truncation at quadratic order in the response field, it is here exact in the large $|\vec{p}|$ limit because of the symmetries.

[115] This holds true in the presence of the regulator $R_{\kappa}$, and would not without it. 